\title{
The Effect of Embedded Lies on the Verbal Quality of Statements and Implications for Credibility Assessment
}

Citation for published version (APA):

Verigin, B. (2020). The Effect of Embedded Lies on the Verbal Quality of Statements and Implications for Credibility Assessment. [Doctoral Thesis, Maastricht University]. https://doi.org/10.26481/dis.20200622bv

Document status and date:

Published: 01/01/2020

DOI:

10.26481/dis.20200622bv

Document Version:

Publisher's PDF, also known as Version of record

\section{Please check the document version of this publication:}

- A submitted manuscript is the version of the article upon submission and before peer-review. There can be important differences between the submitted version and the official published version of record.

People interested in the research are advised to contact the author for the final version of the publication, or visit the DOI to the publisher's website.

- The final author version and the galley proof are versions of the publication after peer review.

- The final published version features the final layout of the paper including the volume, issue and page numbers.

Link to publication

\footnotetext{
General rights rights.

- You may freely distribute the URL identifying the publication in the public portal. please follow below link for the End User Agreement:

www.umlib.nl/taverne-license

Take down policy

If you believe that this document breaches copyright please contact us at:

repository@maastrichtuniversity.nl

providing details and we will investigate your claim.
}

Copyright and moral rights for the publications made accessible in the public portal are retained by the authors and/or other copyright owners and it is a condition of accessing publications that users recognise and abide by the legal requirements associated with these

- Users may download and print one copy of any publication from the public portal for the purpose of private study or research.

- You may not further distribute the material or use it for any profit-making activity or commercial gain

If the publication is distributed under the terms of Article $25 \mathrm{fa}$ of the Dutch Copyright Act, indicated by the "Taverne" license above, 


\title{
Maastricht University
}

\section{The Effect of Embedded Lies on the Verbal Quality of Statements and Implications for Credibility Assessment}

\author{
DISSERTATION
}

to obtain the degree of Doctor of Philosophy from The University of Portsmouth and the degree of Doctor at Maastricht University, on the authority of the

Rector Magnificus, Prof. dr. Rianne M. Letschert in accordance with the decision of the Board of Deans, to be defended in public on Monday June 22, 2020 at 12.00 hours 


\section{Supervisors:}

Dr. Ewout H. Meijer

Prof. dr. Aldert Vrij

Prof. dr. Marko Jelicic

\section{Doctoral Thesis Committee:}

Prof. dr. Harald Merckelbach (chair)

Dr. Lucy Akehurst

Prof. dr. Eric Rassin

Dr. Anna Sagana

Prof. dr. Dave Walsh
Maastricht University, the Netherlands University of Portsmouth, United Kingdom Maastricht University, the Netherlands

Maastricht University, the Netherlands University of Portsmouth, United Kingdom Erasmus University Rotterdam, the Netherlands Maastricht University, the Netherlands De Montfort University, United Kingdom

This research was supported by a fellowship awarded from the Erasmus Mundus Joint Doctorate Program: The House of Legal Psychology (EMJD-LP) with Framework Partnership Agreement (FPA) 2013-0036 and Specific Grant Agreement (SGA) 2016-1339 to Brianna L. Verigin. 


\section{Table of Contents}

Chapter 1 General Introduction 1

Chapter 2 Lie Prevalence, Lie Characteristics, and Strategies of Self-Reported Good Liars 15

Chapter $3 \quad$ The Interaction of Truthful and Deceptive Information 37

$\begin{array}{lll}\text { Chapter } 4 \quad \text { Embedding Lies into Truthful Stories } & 61\end{array}$

Chapter 5 A Within-Statement Baseline Comparison 83

$\begin{array}{lll}\text { Chapter } 6 & \text { General Discussion } & 98\end{array}$

$\begin{array}{ll}\text { References } & 119\end{array}$

$\begin{array}{ll}\text { Summary } & 142\end{array}$

$\begin{array}{ll}\text { Acknowledgements } & 144\end{array}$

$\begin{array}{ll}\text { Dissemination } & 146\end{array}$

$\begin{array}{ll}\text { Curriculum Vitae } & 147\end{array}$

$\begin{array}{ll}\text { Valorisation Addendum } & 148\end{array}$

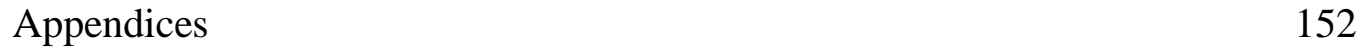




\section{Chapter 1: \\ General Introduction}




\section{General Introduction}

In 2016, two shocking events - the US presidential election and Britain's withdrawal from the European Union - changed the landscape of modern politics. These events also played a role in the rise of the post-truth era. That is, in today's society, objective facts are less influential in shaping public opinion than appeals to emotion and personal belief. Helped by new technology and a declining trust in the media, modern politicians are getting away with a new depth and pervasiveness of deception. From the thousands of fabricated or misleading claims told by Trump during his presidency (Dale, 2019; Rizzo, Kessler, \& Kelly, 2019), to the infamous Leave claims of $£ 350$ million for the National Health Service (Dilnot, 2016; Lichfield, 2017), there is evidence of an upsurge of lies and an erosion of facts and analysis in society as of late. The consequence is a political and social infrastructure that has considerably devalued truth.

Deception is not only relevant to social and political spheres. In the justice system, for instance, errors in detecting deception can have tragic consequences. When the serial rapist Paul Bernardo was first interviewed by police he was asked why he thought he was being investigated for the rapes, he admitted that he resembled the composite sketch of the suspect. After a brief interview, the detectives concluded that such a well-educated, poised and friendly young man could not be responsible for the heinous crimes. They opined that he was "far more credible" than the woman who had reported that he was the rapist; she was described as being awkward, having an unusual way of speaking, and as perhaps only trying to collect the reward (Williams, 1998). Bernardo was released the following day, which allowed him to perpetrate numerous additional rapes and go on to become one of Canada's most infamous serial killers.

With such anecdotes, it is not surprising that the study of deception has garnered the attention of laypeople and scholars alike. In general terms, the purpose of this doctoral thesis was to advance the fields of deception and credibility assessment. More specifically, the main focus of this research was to investigate how the strategic embedding of lies influences the verbal behaviour of liars. In this chapter, I will broadly introduce the importance of studying deception 
detection for investigative purposes. Subsequently, I will discuss the theoretical foundations of verbal deception detection, emphasising the memory-based and strategy-based origins of several prominent verbal cues to deception. My introduction will also extend to the strategies of lying and truth-telling interviewees, wherein I will highlight the strategy of embedding lies. I will then put forward the aims of the present research: (i) to explore the deception strategies of good liars, (ii) to examine the extent to which truthful and deceptive information interact to influence verbal quality, and (iii) to test whether embedded lies can be exploited to facilitate within-statement lie detection. Finally, the research conducted to address these aims will be described.

\section{Importance of Studying Deception}

Determining the credibility of an interviewee, be it a suspect, victim or witness, is a fundamental component of the investigative process. It is therefore not surprising that the scientific study of deception has already spanned over a century (Münsterberg, 1908). Indeed, several hundred studies have measured people's ability to distinguish between truthful and deceptive accounts. The most robust finding in this literature is that humans are poor lie detectors, accurately detecting lies at rates barely exceeding chance level (e.g., Bond \& DePaulo, 2006; Levine, 2010; Vrij, 2008a). This finding also holds true for professionals such as police officers (e.g., Bond \& DePaulo, 2006; Hartwig, Granhag, Strömwall, \& Vrij, 2004; Luke et al., 2016).

One of the explanations for the poor lie detection performance is that there is no single behaviour, nor group of behaviours, systematically and reliably indicative of deception (DePaulo et al., 2003; Hartwig \& Bond, 2011; Wright \& Wheatcroft, 2017). Yet, people continue to base their judgements on stereotypical nonverbal cues such as gaze aversion (Bogaard, Meijer, Vrij, \& Merckelbach, 2016; Strömwall, Granhag, \& Hartwig, 2004).

\section{Verbal Lie Detection}

The most diagnostic information is found in the content of liars' speech. The accuracy of credibility assessments improves when judgements are based only on the verbal content of a statement (52\% correct lie-truth classifications) versus through visual mediums $(63 \%$ correct 
classifications; Bond \& DePaulo, 2006). In fact, a review of over thirty studies showed that when observers' judgements were guided by statement analysis, their accuracy rates increased to $72 \%$ (Vrij, 2005). Additionally, good lie detectors report a higher reliance on verbal cues when making credibility judgments, whereas poor lie detectors tend to rely primarily on non-verbal cues (Mann, Vrij, \& Bull, 2004). It has therefore been advocated that for lie detection purposes, both in research and practice, it is more valuable to listen carefully to what interviewees say than to observe their nonverbal behaviour (Vrij, 2008b).

One of the most consistent findings in the verbal lie detection literature is that liars' statements are typically less richly detailed than those of their truth-telling counterparts (e.g., Amado, Arce, Fariña, \& Vilarino, 2016; DePaulo et al., 2003; Luke, 2019). In fact, the estimated effect size of quantity of details is $d=0.55$ (Amado et al., 2016), which is remarkably high for the social sciences (Funder \& Ozer, 2019; Richard, Bond, \& Stokes-Zoota, 2003). Additionally, meta-analytical findings support the usefulness of temporal, visual, and auditory details for differentiating truthful from false accounts (Masip, Sporer, Garrido, \& Herrero, 2005). Based on these types of variables, the accuracy rate of classifying truth-tellers and liars extends well beyond chance level (Masip et al., 2005; Vrij, Fisher, \& Blank, 2017).

Memory-based cues to deception. The use of content-based details as cues to deception stems from memory research. In particular, two commonly used methods of verbal credibility assessment have their foundations in theories of memory. Reality Monitoring (RM; Johnson \& Raye, 1981) posits that memories derived from real experiences differ in quality from memories based on imagination, due to differences in the cognitive processes involved in externally versus internally generated memories. RM suggests that memories of real events are acquired through perceptual processes and therefore are more likely to contain details relating to sensory, spatial, temporal, and affective information, and to be generally more clear, sharp and vivid, compared to imagined events. Similarly, Criteria-Based Content Analysis (CBCA; Steller \& Köhnken, 1989 ) is based on the hypothesis that memories of personally experienced events differ in both 
quality (e.g., the logical structure or plausibility) and content (e.g., unexpected complications) to memories of fabricated or imagined accounts (Undeutsch, 1967, 1989). Moreover, research supports these theoretical rationales within lie detection contexts: Liars lack the memory traces truth-tellers have, leaving them unable to provide as many rich details as honest individuals (Masip et al., 2005; Oberlader et al., 2016; Vrij, 2008). Though not without criticism, (see Masip et al., 2005; Vrij, 2005), CBCA and RM are favourable tools as, with appropriate training, evaluators' accuracy rates increase from chance level to 65\% - 80\% (Vrij, 2008a).

Strategy-based cues to deception. Some aspects of deception cannot be accounted for solely by the assumption that lies and truths stem from different memory processes. RM does not, for example, consider the intention of liars to strategically manipulate their statements to make them appear truthful (Nahari, 2018a). To supplement the memory-based approach to deception, more recent deception detection techniques aim to exploit interviewees' strategies for appearing credible. One notable example is the Verifiability Approach (VA; Nahari, Vrij, \& Fisher, 2014a, 2014b). The VA works on the assumption that liars, on the one hand, are inclined to provide detailed statements to be perceived as cooperative and credible, but, on the other hand, want to minimise the chances that investigators can falsify their statement (Masip \& Herrero, 2013; Nahari et al., 2014a). A strategy that meets these contradictory aims is to provide information that cannot be verified.

Another example of a deception detection measure that is sensitive to the verbal strategies of liars and truth-tellers relates to common knowledge details (i.e., strongly invoked stereotypical information about events; Vrij, Leal, Mann et al., 2017). Whereas truth-tellers have personal, unique experiences of an event (DePaulo, Kashy, Kirkendol, Wyer, \& Epstein, 1996), liars typically lack such information and their reports tend to be characterised by more general, impersonal knowledge (Sporer, 2016). Liars also tend to report fewer complications occurrences that make a situation more difficult than necessary, often characterised by disrupted activity or failing efforts (e.g., missing the bus; Steller \& Köhnken, 1989; Vrij, Leal, Jupe, \& 
Harvey, 2018) - compared to truth-tellers. This makes sense given liars' preference for simple stories (Hartwig et al., 2007). An additional strategy-based measure relates to self-handicapping strategies (i.e., justifications as to why someone is unable to provide certain information; Vrij, Leal, Mann et al., 2017). Liars are motivated to keep their stories simple; however, they also realise that admitting a lack of knowledge and/or memory may generate suspicion from investigators (Ruby \& Brigham, 1998). A strategic solution then is to provide a justification for the failure to provide information. Taking these three cues together, investigators can calculate the proportion of complications (complications / complications + common knowledge details + self-handicapping strategies), which represents the proportion of cues to truthfulness. This proportion score has theoretical advantages given that it is a within-subjects comparison that is also sensitive to the different verbal strategies used by liars and truth-tellers (Vrij, Leal, Jupe, \& Harvey, 2018).

\section{Liars' and Truth-Tellers' Strategies}

Strategy-based deception detection techniques derive from a larger body of research on interviewees' counter-interrogation strategies. This work is premised on the idea that it may be possible to identify more reliable deception cues, and potentially improve lie detectors' ability to discriminate between liars and truth-tellers, by improving our understanding of how liars and truth-tellers try to tell a convincing story (e.g., Clemens, 2013). The fact that individuals, guilty or innocent, adopt particular strategies in their attempts to appear credible can be explained by the theory of self-regulation: A social cognitive framework for understanding how people control their behaviour to move away from undesired outcomes and to reach desired goals (Carver \& Scheier, 2012; Clemens, Granhag, \& Strömwall, 2013; Fiske \& Taylor, 2008; Hartwig, Granhag, \& Luke, 2014). In legal contexts, this translates to a common goal among liars and truth-tellers, who both aim to convince the interviewer of their credibility. Importantly, the information management strategies by which they attempt to reach this goal will differ, largely due to 
differences in the mental state of liars and truth-tellers (Hartwig, Granhag, Strömwall, \& Doering, 2010).

Embedded lies. One strategy that may contribute to liars' success is the embedding of lies into truthful information. Embedded lies can be defined as fragments of deceptive information integrated into truthful stories designed to mislead an investigator. When given the opportunity, liars will incorporate such embedded lies into their accounts. Evidence for the prevalence of embedded lies comes from Leins, Fisher, and Ross (2013). In two studies, these authors found that the majority of liars $-67 \%$ in the first study and $86 \%$ in the second - chose to formulate their deceptive account based on a previously experienced event. Additionally, in their study evaluating the verifiability approach, Nahari et al. (2014b) found that of their 44 mock criminals who were instructed to provide deceptive statements, more than half indicated that over $20 \%$ of their statement was truthful.

Further evidence comes from Hartwig and colleagues' (2007) examination of guilty and innocent suspects' strategies, which revealed that one of liars' most endorsed strategies was to avoid lying by telling the truth as much as possible. In fact, liars will generally provide as much experienced or truthful information as possible, to the extent that they do not incriminate themselves (Nahari \& Vrij, 2015). The use of embedded lies has been observed across various populations, such as research participants (Bell \& DePaulo, 1996; Leins, Zimmerman, \& Polander, 2017), non-criminals engaging in deception (DePaulo et al., 2003), and even criminal suspects (Strömwall \& Willén, 2011). It has been speculated that this strategy can enhance one's credibility because lies of this type may be easier to deliver and more difficult for interviewers to detect, as compared to outright lies (Vrij, 2008a).

\section{New Research Directions}

Nearly all research to date treats deception as dichotomous. That is, statements are classified as either truthful or deceptive, and participants as either liars or truth-tellers. This division is reflected in hypotheses, designs and experimental procedures (e.g., McCornack, 
Morrison, Paik, Wisner, \& Zhu, 2014). Some researchers have acknowledged that liars prefer to embed lies into otherwise truthful statements (e.g., Leins et al., 2013; Nahari 2018a; Nahari \& Vrij, 2015; Vrij, 2008a; Vrij, Granhag, \& Porter, 2010), though this has mostly been in the form of disclaimers within discussion sections. Few have explicitly acknowledged, nor systematically manipulated, the inclusion of both truthful and deceptive information within statements. Doing so would more accurately reflect real world conditions in which suspects interweave truths and lies (e.g., Leins et al., 2017; Strömwall \& Willén, 2011; Vrij, 2008a).

There is variation in the extent to which deceptive information can be integrated into truthful stories. For instance, an arsonist who denies having set a fire yesterday can instead claim that he visited the gym. He can then describe an entirely truthful recollection of a previous visit to the gym, lying only about the day and time at which the visit occurred. It is also possible that liars embed more elaborate descriptions of deceptive events into their statements. For example, when providing an alibi statement, a burglar could report the majority of his day truthfully, but could lie about his activities during the window of time during which he committed the burglary. This latter type of embedded lie, whereby liars integrate descriptions of deceptive events into otherwise truthful stories, is the focus of the current thesis.

As it stands, it remains unknown how and to what extent such embedded lies affect the verbal content of statements and what implications this may have for verbal lie detection tools. This thesis aimed to address these gaps. Our objectives were threefold. First, we aimed to advance our understanding of liars' deception strategies, particularly those that help self-reported good liars evade detection. Second, we aimed to narrow in on how the strategic embedding of lies may affect statement quality. Third, we aimed to work towards a within-statement method for exploiting embedded lies to improve deception detection ability. These lines of inquiry are both highly warranted and long overdue, and will provide critical insights for credibility assessors in both research and practice. What follows is a list of topics directly related to the scope of this thesis that warrant further investigation. 
Good liars. Most studies have focused either on strategies and cues designed to improve discriminatory accuracy, or individual differences in lie detection ability (Vrij \& Granhag, 2012). A growing body of evidence, however, indicates that the accuracy of detecting deception depends more on the skill or characteristics of the deceiver and less on the judges' lie detection ability (Bond \& DePaulo, 2008; Bond, Kahler, \& Paolicelli, 1985; Law et al., 2018; Levine, 2016; Levine et al., 2011). Even so, only a handful of studies have attempted to determine individual differences in the ability to lie credibly (e.g., DePaulo \& Rosenthal, 1979; Riggio, Tucker, \& Throckmorton, 1987; Vrij, Granhag, \& Mann, 2010; Wright, Berry \& Bird, 2012, 2013).

Little is known about what constitutes good liars, or what causes the variation in detectability. Some research, for example, has shown that sender demeanor explained up to $98 \%$ of the variance in detection accuracy (Levine et al., 2011). Other researchers have speculated that good liars use effective strategies to conduct their behaviour, by attempting to act in line with people's beliefs about how truth-tellers behave while avoiding behaviour associated with liars (Vrij, Granhag, \& Mann, 2010). Still, this field is in its infancy and additional research on what characterises those who escape detection (i.e., good liars) would be highly beneficial in investigative settings. In particular, digging deeper into good liars' strategies could aid in the development of strategy-based interventions targeted at these individuals, who may be most likely to avoid detection.

Embedded lies and the effect on statement quality. Research on beliefs about cues to deception and liars' strategies suggests that the embedding of lies could affect their quality. Laypeople and legal professionals alike believe that inconsistency is symptomatic of deception (Blair, Reimer, \& Levine, 2018; Vredeveldt, van Koppen, \& Granhag, 2014; Strömwall \& Granhag, 2003). Accordingly, one of the main concerns of liars - and one of their most frequently reported strategies - is to maintain consistency (Deeb, Vrij, Hope, Mann, Granhag, \& Lancaster, 2017; Hartwig, Granhag, Strömwall, \& Doering, 2010). Moreover, liars tend to be more concerned with reducing the amount of within-statement inconsistency (i.e., inconsistencies 
between details within a single statement) than any other type of inconsistency, such as betweenstatement inconsistency (i.e., the level of consistency between different statements made by the same suspect) or statement-evidence inconsistency (i.e., the level of consistency between the suspect's statement and the other evidence; Deeb et al., 2017, 2018). In one of the only studies to include statements that purposefully contained both truths and lies, Deeb et al. (2017) instructed liars to provide a statement containing reports about two events, one deceptive and the other truthful, during two interviews. When asked about their strategies for appearing credible, $45 \%$ of liars mentioned maintaining consistency for both events between statements. Moreover, many liars reported that they did so by strategically lowering their "baseline consistency" by including fewer repetitions when they reported truthfully to match the number of repetitions provided during their deceptive report. A plausible explanation is that liars do not want the deceptive component of their story to stand out from the remainder of their statement, as that would draw attention to their lies. Thus, if interviewees embed their lies in an otherwise truthful statement, their efforts to maintain consistency may also extend to the quality of details provided between the truthful and deceptive parts of their statement.

If liars are able to successfully maintain consistency regarding the quality of details provided between the lies and truths of their statement, it could mean that lies become, for example, more richly detailed when surrounded by truths. Embedded lies that are characterised by high quality contextual and perceptual details could jeopardise the diagnostic accuracy and utility of verbal credibility assessment tools such as CBCA and RM, that are premised upon differences in the content of accounts based on experienced versus non-experienced events (e.g., Masip et al., 2005; Vrij, 2005; Vrij, Granhag, \& Porter, 2010). This could be particularly problematic in investigative contexts given that guilty suspects who strategically embed their lies may go undetected by these tools.

Despite concerns, little research has addressed how lies that are embedded into truthful accounts may differ from lies that are completely fabricated. One study that included 
comparisons between partially deceptive and truthful mock suspects found that partially deceptive statements had fewer details and were less coherent than truthful statements (Porter \& Yuille, 1996). The authors speculated that the act of lying affected the quality and quantity of information provided, as opposed to the memories themselves. In fact, even when liars and truthtellers carry out the same activities (but for either malevolent or benevolent reasons), verbal differences still occur with liars reporting their activities in less detail than truth-tellers (Vrij, Mann, Jundi, Hillman, \& Hope, 2014). Additionally, research by Palena and colleagues (2018) required interviewees to tell the truth about one theme (event) but to lie about another, during one interview. Their results showed that truth-tellers reported the same amount of information about both themes, whereas liars reported fewer details for the theme they lied about compared to the theme they reported truthfully.

Few previous studies have also examined how lies differ when they are invented or based on past experiences, and to what extent this influences verbal lie detection tools. For instance, one previous study found that the content of liars' and truth-tellers' statements differed on RM criteria even when past experiences were used, but that RM was most effective when liars invented their stories rather than using previous experiences (Gnisci, Caso \& Vrij, 2010). Similar results were also found in unpublished work by Valois and colleagues (2019). These authors examined whether $\mathrm{CBCA}$ and $\mathrm{RM}$ techniques could differentiate both fully and partially deceptive accounts from truthful accounts, finding that both tools were still effective even when interviewees incorporated truthful events into fabricated accounts. Given the scarcity of work on this topic, further research is warranted to understand the effects of embedded lies on statement quality and the subsequent consequences for the reliability of verbal credibility assessment tools. Such research could inform both researchers and practitioners whether or not the commonly used methods of credibility assessment (e.g., CBCA and RM) are robust against the embedding of deceptive events into otherwise truthful stories. 
Within-statement lie detection. On the one hand, embedded lies may pose a risk to the reliability of verbal credibility assessment tools, but on the other hand, lie detectors may be able to exploit these lies to benefit deception detection. One way to do so is by using the baseline technique. Baselining refers to the practice in which interviewers evaluate the veracity of a critical component of a statement relative to a baseline, or neutral, component of the interview (see Vrij, 2016 for an overview), and is reportedly used by some police in practice (Ewens, Vrij, Jang, \& Jo, 2014; Frank, Yarbrough, \& Ekman, 2006; Inbau et al., 2013; U.S. Department of the Army, 2006). Previous research on baselining shows that to enhance diagnostic accuracy, the baseline statement must be equivalent to the statement of interest in terms of content, time-frame, stakes, cognitive and emotional involvement, and questioning context (i.e., a comparable truth baseline; Caso, Palena, Vrij, \& Gnisci, 2019; Ewens et al., 2014; Palena, Vrij, Caso, \& Orthey, 2018). Moreover, a comparable truth baseline can enhance truth-lie discrimination accuracy in some contexts (e.g., Caso, Palena, Vrij, \& Gnisci, 2019).

It is also possible that, if portions of an interviewee's statement can be verified as truthful, embedded lies could provide an opportunity for a within-statement baseline comparison. Investigators could theoretically derive a baseline statement from parts of an interviewee's statement to successfully increase deception accuracy. This would have important implications for practitioners who may be inclined to draw such comparisons between corroborated and uncorroborated portions of an interviewee's account. Within-statement comparisons also facilitate veracity decisions at the individual level, thereby overcoming common criticisms of deception research relating to generalisations of group-derived estimates to individual cases (e.g., Fisher, Medaglia, \& Jeronimus, 2018). It is therefore unsurprising that researchers and practitioners alike have called for more research of this type (e.g., Vrij, 2016).

\section{Aim and Outline of the Current Thesis}

This $\mathrm{PhD}$ thesis provides an empirical examination of embedded lies, with the aim of answering three central questions: (i) which strategies do people who claim to be good liars report 
using to successfully evade detection, (ii) to what extent do embedded lies affect the verbal quality of statements, and (iii) can embedded lies be exploited to facilitate lie detection? To answer these questions, a mixed-methodological approach was taken. Chapter 2 reports survey research examining the deception strategies of self-reported good liars, whereas Chapters 3, 4 and 5 contain experimental research testing the impact of embedded lies on the verbal quality of statements and the potential for exploiting embedded lies using a within-statement lie detection approach. Additionally, in the general discussion (Chapter 6), an overview of the key findings is presented, followed by the theoretical and practical implications of our results for credibility assessments in academic and applied contexts. The studies are summarised in further detail below.

Chapter 2 (Study I). Meta-analyses indicate that the success of unmasking a deceptive interaction relies more on the performance of the liar than on that of the lie detector. However, the lie characteristics and strategies of deception that enable good liars to evade detection are largely unknown. We conducted a survey to explore the association between laypeople's selfreported ability to deceive and their lie frequency, characteristics, and deception strategies in daily life. Our goal was to provide insight into what characterizes those who escape detection, i.e., good liars, which would be highly beneficial in investigative settings.

Chapter 3 (Study II). Although it has been acknowledged that liars prefer to embed lies into otherwise truthful statements, there is a scarcity of research on how truthful and deceptive information may interact within statements. We experimentally tested how interviewees strategically regulate the information they provide when their accounts contain both truths and lies. Specifically, participants provided one statement consisting of two elements. We manipulated the veracity of these elements, with participants either delivering both truthful, both deceptive, or one truthful and the other deceptive elements. Our foremost objective was to investigate how participants maintain verbal consistency within their accounts. We hypothesized that they may do so by calibrating the content of the second element of their statement to that of 
the first element. This would imply that elements preceded by a lie would be less detailed than elements preceded by a truth. Also conceivable was that participants would anticipate the second element, and calibrate the content of the first element to that of the second. Accordingly, we also hypothesized that elements followed by a lie would be less detailed than those followed by a truth.

Chapter 4 (Study III). The extent to which embedded lies hinder the effectiveness of verbal credibility tools is largely unknown. In this study, we extended our paradigm to incorporate lies that were embedded into truthful information, to examine whether lies that were embedded into truthful stories were qualitatively different than lies that were part of entirely fabricated statements. Participants provided a statement that was either entirely truthful, entirely fabricated, or had the fabricated element of interest embedded into an otherwise truthful story. We tested for differences in the richness of detail, as well as differences in a number of other content cues (e.g., verifiability of information, proportion of complications), (i) between the completely truthful statements compared to the completely deceptive statements, and (ii) between lies embedded into otherwise truthful statement compared to lies embedded into an entirely fabricated statement.

Chapter 5 (Study IV). Finally, we examined whether embedded lies could be exploited as a within-statement baseline comparison to facilitate lie detection. Participants read a mock suspect's alibi statement and provided a veracity judgement regarding a critical two-hour period within the statement. This critical element was either deceptive or truthful and was embedded into an otherwise truthful story. We examined if providing an instruction to utilize a comparable baseline (i.e., informing participants that all information, with the exception of the critical statement, has been confirmed to be truthful) could enhance judges' detection accuracy. We hypothesized that participants who received the baseline instruction would have more accurate veracity judgements than participants who did not receive the baseline instruction. 


\section{Chapter 2: Lie Prevalence, Lie Characteristics}

\section{and Strategies of Self-Reported Good Liars}

This chapter was published as:

Verigin, B. L., Meijer, E. H., Bogaard, G., \& Vrij, A. (2019). Lie prevalence, lie characteristics and strategies of self-reported good liars. PLOS ONE, 14, e0225566, doi: 10.1371/journal.pone.0225566 


\begin{abstract}
Meta-analytic findings indicate that the success of unmasking a deceptive interaction relies more on the performance of the liar than on that of the lie detector. Despite this finding, the lie characteristics and strategies of deception that enable good liars to evade detection are largely unknown. We conducted a survey $(n=194)$ to explore the association between laypeople's selfreported ability to deceive on the one hand, and their lie prevalence, characteristics, and deception strategies in daily life on the other. Higher self-reported ratings of deception ability were positively correlated with self-reports of telling more lies per day, telling inconsequential lies, lying to colleagues and friends, and communicating lies via face-to-face interactions. We also observed that self-reported good liars highly relied on verbal strategies of deception and they most commonly reported to (i) embed their lies into truthful information, (ii) keep the statement clear and simple, and (iii) provide a plausible account. This study provides a starting point for future research exploring the meta-cognitions and patterns of skilled liars who may be most likely to evade detection.
\end{abstract}




\section{Introduction}

Despite the importance of being able to detect deception, research has consistently found that people are unable to do so. In fact, the accuracy rates vary around chance level (e.g., Bond \& DePaulo, 2006; Vrij, 2008). Lacking good lie detectors, a growing body of evidence indicates that the accuracy of detecting deception depends more on the characteristics of the liar and less on the lie detection ability of the judge (Bond \& DePaulo, 2008; Bond, Kahler, \& Paolicelli, 1985; Law et al., 2018; Levine, 2016; Levine et al., 2011). The meta-analysis of Bond and DePaulo (2008) provided robust evidence that liars vary in their detectability. Their analysis showed that differences in detectability from sender to sender are more reliable than differences in credulity from judge to judge, with reliability coefficients of .58 and .30 , respectively. This pattern of results was replicated by Law et al. (2018), lending support to the proposition that liar characteristics exert a powerful influence on lie detection outcomes. Moreover, Levine et al. (2011) showed that sender demeanour explained up to $98 \%$ of the variance in detection accuracy.

Yet, only a handful of studies have attempted to determine individual differences in the ability to lie credibly (e.g., DePaulo \& Rosenthal, 1979; Riggio, Tucker, \& Throckmorton, 1987; Vrij, Granhag, \& Mann, 2010; Wright, Berry \& Bird, 2012, 2013). Research on what characterises those who escape detection, i.e., good liars, would be highly beneficial in investigative settings. Thus, a focus on the liar, in particular the skilled liar, was the aim of this study. Specifically, the present chapter reports an exploratory study addressing how self-reported deception ability is associated with lie prevalence and lie characteristics, and how self-reported good liars utilise strategies for deceiving.

First, we investigated the relationship between liars' self-reported lie-telling frequency and self-reported deception ability. The most widely cited research on deception prevalence estimates the frequency at an average of once or twice per day (DePaulo, Kashy, Kirkendol, Wyer, \& Epstein, 1996; see also George \& Robb, 2008). More recent research, however, shows 
that the distribution of lies per day is considerably skewed. The majority of lies are told by only a handful of prolific liars (Serota \& Levine, 2015; Serota, Levine, \& Boster, 2010; Halevy, Shalvi, \& Verschuere, 2014). Specifically, Serota and Levine (2015) showed that in almost 3,000 participants, $5 \%$ of people accounted for over $50 \%$ of all the lies reported within the past 24 hours, whereas the majority of subjects reported telling no lies at all. Several additional studies, as well as a reanalysis of the DePaulo et al.'s (1996) diary study, have validated that the majority of lies are told by a minority of people (e.g., George \& Robb, 2008; Serota et al., 2010). These few prolific liars tend to tell more serious lies that carry significant consequences if detected (Serota \& Levine, 2015). Also, people who self-reported to lie more often were more prone to cheating in laboratory tasks for personal profit (Halevy et al., 2014). It is possible that these prolific liars also perceive themselves as more skilled at deceiving and tell more lies that they think will stay undetected, either because they believe the receiver will not try to find out or they believe they are good enough to fool the receiver.

Second, our investigation examined whether characteristics of lies differ as a function of self-reported deception ability. The first of these characteristics is the type of lie. This can refer to the severity; at one end are white lies, which are relatively common (Feldman, Forrest, \& Happ, 2002) and often used to ease social interactions (e.g., telling your mother-in-law that her baking is delicious when you actually dislike sweets; Vrij, 2007), while at the other end are boldfaced fabrications, which are less common and typically serve to protect the liar (e.g., denying having had an affair; Craig, 2003). The latter type of lies are also encountered more by the legal system (Vrij, Edward, Roberts, \& Bull, 2000). Other taxonomies of lies also exist, for example lies of omission or lies embedded into the truth; however, research has yet to explore how the types of lies could differ as a function of deception ability. Is it that, for example, good liars tend to utilise a certain type of lie which facilitates their success? The second characteristic is the receiver of the lie. Lies can be communicated to a variety of individuals ranging from family, 
romantic partners, and friends to strangers, colleagues, or authority figures. Previous research has shown that people lie less frequently in close relationships than in casual relationships (DePaulo \& Kashy, 1998). A third characteristic we are interested in is the medium of deception, as this can also influence the success of one's lie. Some liars, for instance, prefer online communication (Van Swol, Braun, \& Kolb, 2015). This would fit the liars' (erroneous) belief that their deception will leak out via behavioural cues (Vrij, Granhag, \& Porter, 2010). It is unknown, however, if or how good liars concentrate their lies to specific individuals or communicate via certain mediums.

Finally, we examined how self-reported good liars utilise strategies for deceiving. The idea that liars adopt strategies to enhance the likelihood of successfully deceiving stems from research on impression and information management. Both forms of regulation relate to the idea that much of social behaviour is controlled for the purpose of interpersonal presentation (DePaulo, 1992; DePaulo et al., 2003). In legal contexts, both liars and truth-tellers are motivated to achieve a favourable impression and attempt to do so by regulating their speech and behaviour, albeit liars more so than truth-tellers (e.g., Hartwig, Granhag, \& Strömwall, 2007). The topic of deceivers' strategies has received some empirical attention (e.g., Colwell, Hiscock-Anisman, Memon, Woods, \& Michlik, 2006; Hartwig et al., 2007; Hartwig, Granhag, Strömwall, \& Doering, 2010; Strömwall, Hartwig, \& Granhag, 2006). For example, it was found that among the principal strategies of criminal offenders were "Staying close to the truth," and "Not giving away information" (Strömwall, \& Willén, 2011). Researchers have also capitalised on this increased awareness of liars' and truth-tellers' strategies by developing strategy-based lie detection tools. For instance, the Verifiability Approach (VA; Nahari, Vrij, \& Fisher, 2014a, 2014b) exploits liars' strategy of providing detailed statements that are embellished with unverifiable information. Still, surveying expert liars as a source of insight into real world deception remains a highly underdeveloped research avenue (e.g., Nahari et al., 2019). 
The purpose of this survey was to shed light on the association between self-reported deception ability and lie prevalence and lie characteristics. Given the exploratory nature of this research objective, we did not specify a-priori hypotheses.

\section{Method}

This study was approved by the ethical committee of the Faculty of Psychology and Neuroscience at Maastricht University. Participants read and signed the informed consent in accordance with the Declaration of Helsinki.

\section{Participants}

The sample consisted of 194 participants (97 females; 95 males; 2 preferred not to say; $M_{\text {age }}=39.12$ years, $S D_{\text {age }}=11.43$ years) recruited via Amazon Mechanical Turk (mTurk). Most participants reported being U.S. citizens $(n=175)$, whereas the remainder $(n=19)$ reported Indian citizenship. Participants who completed the study were paid 1.75 USD. Participants could participate in the study if they reported to be able to understand and write English at an advanced level. To ensure data quality, participants were required to have the mTurk Masters Qualification that is awarded to those who have demonstrated continual excellence across a wide range of mTurk projects. An additional 133 participants began the questionnaire but did not complete it, therefore their data were discarded. Data from nine participants were also removed because of insufficient responses. We reached our sample size $(n=194)$ after these exclusions.

\section{Procedure}

The online questionnaire was created on Qualtrics online platform. After providing informed consent, participants were provided definitions of lying and deception modelled from previous research (DePaulo et al., 1996; Vrij, 2008a; see Appendix 1). Participants were asked to read these definitions carefully and to consider them while making responses throughout the questionnaire. In the first part of the questionnaire, participants reported their experience with telling lies in daily life. Participants rated on a 10 -point Likert scale (1 - very poor to $10-$ 
excellent) "How good are you at successfully deceiving others (i.e., getting away with lies)?" Next, they reported the estimated number of lies told during the past 24 hours. Participants then responded to multiple-response questions about (i) the types of lies told during the past 24 hours (options: white lies, exaggerations, lies of omission/concealment, lies of commission/fabrications, embedded lies; see Appendix 1 for the definitions provided to participants); (ii) the receivers of their deception (options: family, friend, employer, colleague, authority figure, or other); and (iii) the mediums of their deception (options: face-to-face, over the phone, social media, text message, email, or other).

The second part of the questionnaire probed the deceivers' strategies. Participants provided an open-ended response to explain "In general, what strategy or strategies do you use when telling lies?" They were then asked to rate on a 10-point Likert scale (1 - not important to 10 - very important) how important they consider verbal strategies of deception and nonverbal strategies of deception to be for getting away with lies (for the definitions provided to participants, see Appendix 1). Finally, participants indicated which verbal strategies they use when telling lies in general from a predetermined set (options: reporting from previous experience, providing details the person cannot check [i.e., unverifiable details], telling a plausible story, etcetera). The options included in this list were drawn from empirical findings regarding liars' strategies and cues to deception (e.g., DePaulo et al., 2003; Leins, Fisher, \& Ross, 2013; Nahari et al., 2014a) ${ }^{1}$. After rating the predetermined set of strategies, participants were unable to modify their previous open-ended responses. Participants then provided demographic information regarding their age, sex, citizenship, ethnicity and education ${ }^{2}$. We explored the

\footnotetext{
${ }^{1}$ Given the theme of this doctoral research, I was particularly interested in how liars might maintain consistency in their statements if they chose to interweave lies and truths. To tease apart how individuals could manipulate their statements to maintain consistency, I included two categories related to the strategic matching of the type and amount of details between lies and truths within statements.

${ }^{2}$ An additional section of the questionnaire probed laypeople's opinions on the types of lies and strategies used by guilty and innocent suspects in an investigative context. Given the conceptual differences, this section was retained for use in a separate manuscript beyond this thesis.
} 
association between laypeople's self-reported deception ability and their sex and education level. Finally, an additional part of the questionnaire asked participants to recall a time in which they had told a serious lie and to report their rationale for lying and their strategies. We examined how the deception rationale influenced their motivation, preparation, strategies, and perceived success of the lie. To conserve manuscript length, the final section of the questionnaire is reported in Appendix 1.

Qualitative analysis. To code the participants' self-reported strategies into data-driven categories, the Principal Investigator performed a content analysis on the open-ended responses to the question probing their use of strategies. First, each participant's strategy or strategies was identified, then all overlapping responses were combined, and these strategies were condensed into several dominant categories with theoretical similarities (i.e., relating to behavioural control or verbal control, etcetera). The Principal Investigator completed each stage of this process and all authors approved upon the final categories. Seven categories emerged from this coding method, for example omitting certain information, relating to truthful information, or controlling behaviour (see Table 2.1).

To establish inter-rater reliability, the main coder and a second coder coded a randomly selected $20 \%$ of the participants' open-ended responses into the appropriate categories. A twoway mixed effects model measuring consistency (e.g., Koo \& Li, 2016) showed that raters were highly consistent across all categories (Single Measures ICCs ranged from .79 to 1.00). After confirming that the raters were consistent, the main coder (Principal Investigator) completed the remaining sample of participant responses and only these scores were used in the analysis.

\section{Results}

We were interested in (i) replicating previous findings regarding the distribution skewness of lie-telling frequency and exploring how these patterns relate to self-reported 
deception ability; (ii) isolating lie characteristics as a function of deception ability; and (iii) exploring the strategies of deception used by self-reported good liars.

\section{Lie Prevalence and Characteristics}

We investigated how laypeople lie in daily life by examining the frequency of lies, types of lies, receivers and mediums of deception within the past 24 hours. Overall, participants indicated telling a mean of 1.61 lies during the last 24 hours $(S D=2.75$; range: $0-20$ lies $)$, but the distribution was non-normally distributed, with a skewness of $3.90(S E=0.18)$ and a kurtosis of $18.44(S E=0.35)$. The six most prolific liars, less than $1 \%$ of our participants, accounted for $38.5 \%$ of the lies told. Thirty-nine percent of our participants reported telling no lies. Figure 2.1 displays participants' lie-telling prevalence.

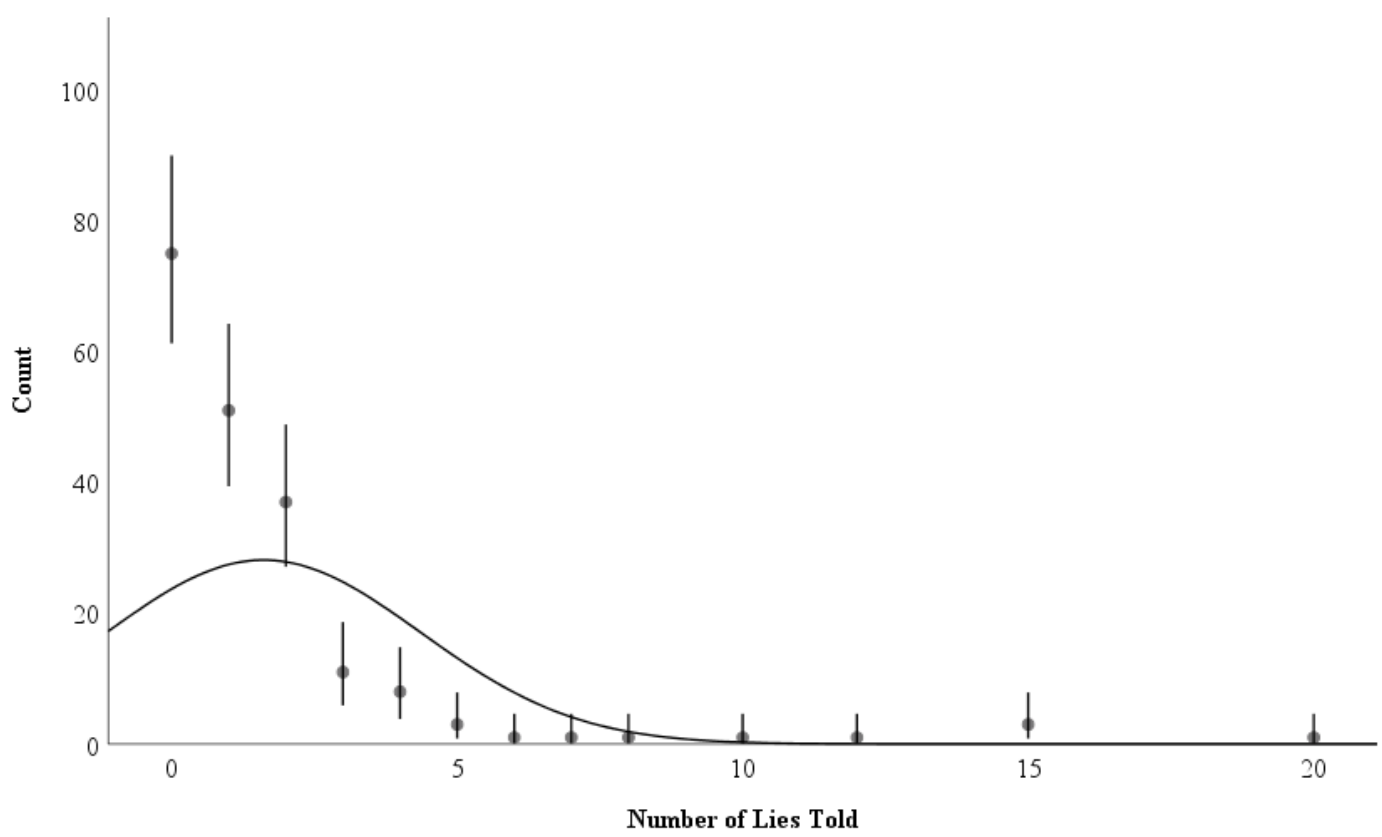

Figure 2.1. Scatterplot of participants' self-reported lie-telling frequency during the past 24 hours. The distribution curve represents the mean and standard deviation of the total sample. Error bars represent $95 \%$ confidence intervals.

Participants' endorsement of the type, recipient, and medium of their lies are shown in Figure 2.2. Participants mostly reported telling white lies, to family members, and via face-toface interactions. All lie characteristics displayed non-normal distributions (See Appendix 1 for the complete description). 

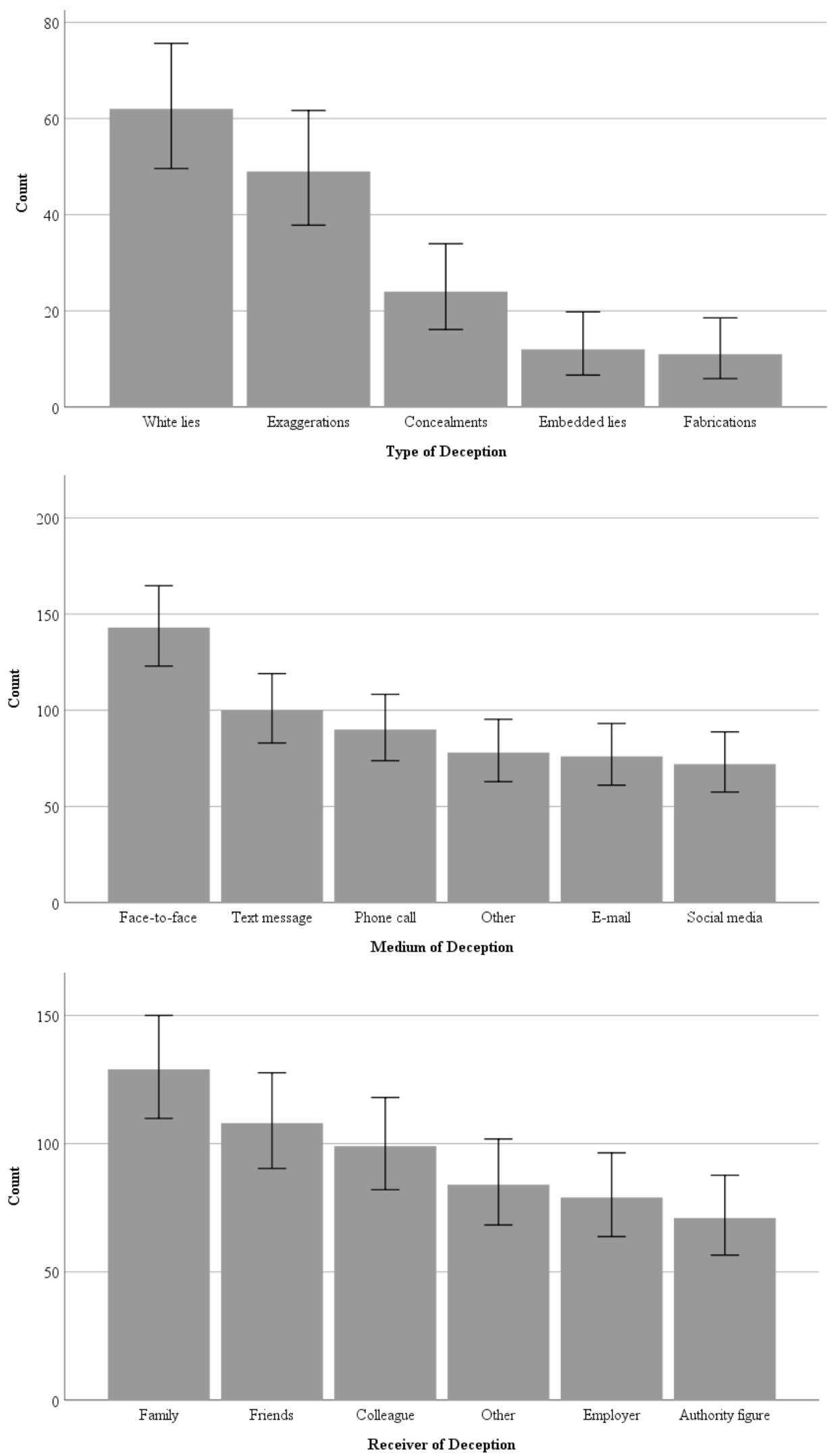

Figure 2.2. Bar charts displaying the frequency of the types, receivers, and mediums of deception endorsed by participants for their reported lies during the past 24 hours. Error bars represent $95 \%$ confidence intervals. For deception recipients, "other" refers to individuals such as intimate partners or strangers; for deception mediums, "other" refers to online platforms not included in the provided list. 
Lie prevalence and characteristics as a function of deception ability. Next, we conducted correlational analyses to examine the association of our participants' lie frequency and characteristics with their self-reported deception ability. An increase in self-reported ability to deceive was positively correlated to a greater frequency of lies told per day, $r(192)=.22, p=$ .002 , and with higher endorsement of telling white lies and exaggerations within the last 24 hours $(r(192)=.16, p=.023$ and $r(192)=.16, p=.027$, respectively). There were no significant associations between self-reported deception ability and reported use of embedded lies, $r(192)=$ $.14, p=.051$; lies of omission, $r(192)=.10, p=.171$; or lies of commission, $r(192)=.10, p=$ .161. Higher self-reported deception ability was significantly associated with telling lies to colleagues, $r(192)=.27, p<.001$, friends, $r(192)=.16, p=.026$, and "other" receivers of deception, $r(192)=.16, p=.031$; however, there were no significant associations between selfreported ability to lie and telling lies to family, employers, or authority figures $(r(192)=.08, p=$ $.243 ; r(192)=.04, p=.558$; and $r(192)=.11, p=.133$, respectively). Finally, higher values for self-reported deception ability were positively correlated to telling lies via face-to-face interactions, $r(192)=.26, p<.001$. All other mediums of communicating the deception were not associated with a higher reported ability, as follows: Via phone conversations, text messaging, social media, email, or "other" sources $(r(192)=.13, p=.075 ; r(192)=.13, p=.083 ; r(192)=$ $.03, p=.664 ; r(192)=.05, p=.484 ; r(192)=.10, p=.153$, respectively $).$

\section{Deception Strategies of Self-Reported Good Liars}

We were also interested in exploring the strategies of deception, particularly those of good liars. To test this, we created categories representing participants' self-reported deception ability, using their scores from the question asking about their ability to deceive successfully, as follows: Scores of three and below were combined into the category of "Poor liars" $(n=51)$; scores of 4, 5, 6, and 7 were combined into the category of "Neutral liars" $(n=75)$; and scores of eight and above were combined into the category of "Good liars" $(n=68)$. 
Table 2.1 provides an overview of the exact values regarding the endorsement of each deception strategy that emerged from the qualitative coding. To examine whether there were associations between the reported strategies and varying deception abilities, we conducted a series of chi square tests of independence on participants' coded responses to the question regarding their general strategies for deceiving. We did not observe any statistically significant associations between self-reported deception ability and the endorsement of any strategy categories (see Table 2.1), apart from one exception. We observed a significant association between Poor, Neutral and Good liars and the endorsement of using "No strategy". Pairwise comparisons were performed using Dunn's (1964) procedure with a corrected alpha level of .025 for multiple tests. This analysis revealed a significant difference in endorsing "No strategy" only between the Good and Poor liars, $p=.004$. However, we did not meet the assumption of all expected cell frequencies being equal to or greater than five and as such these data may be skewed. Based on Cohen's (1988) guidelines, all associations were small to moderate (all Cramer's $V s<.206)$.

Table 2.1

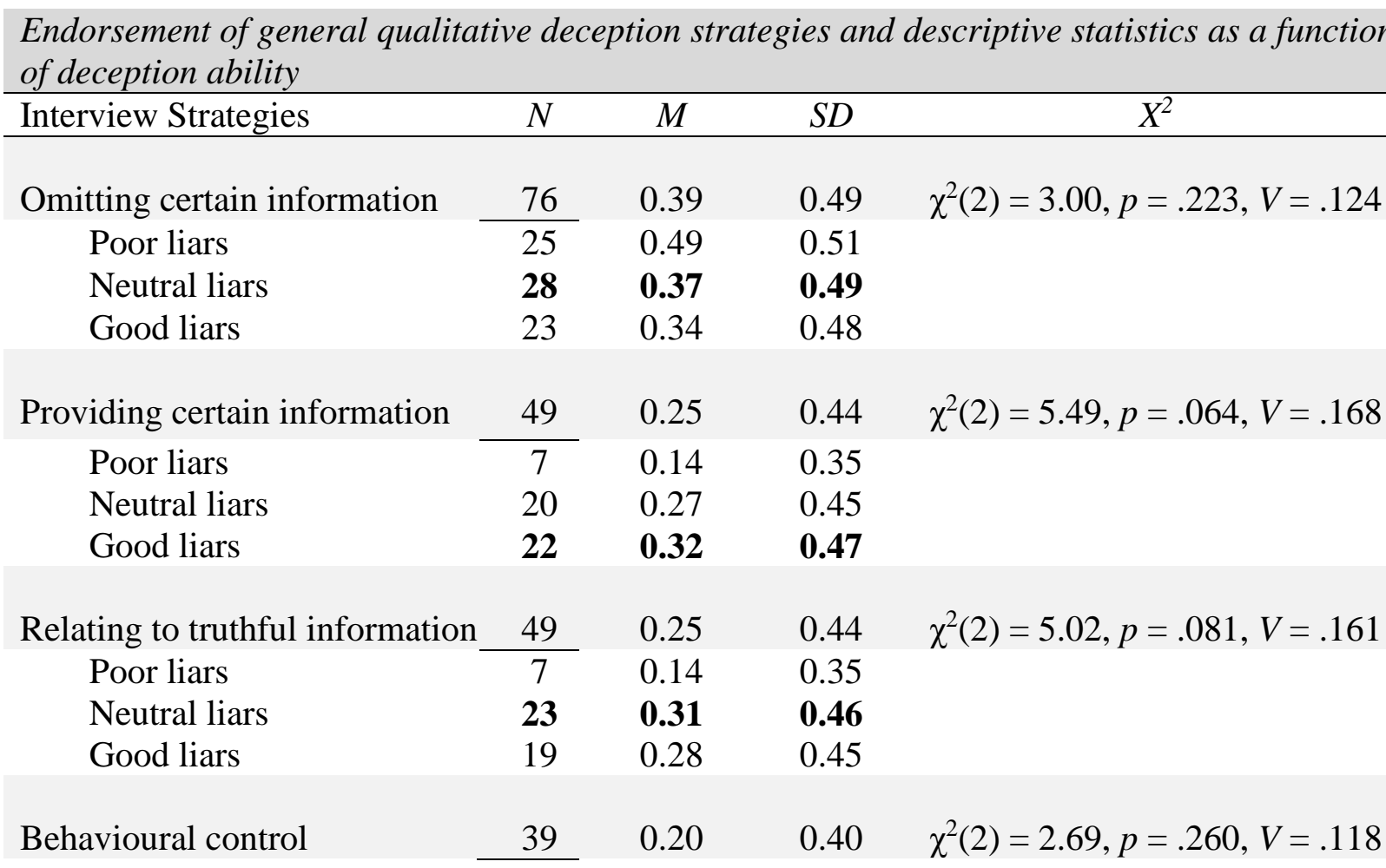




\begin{tabular}{lccc}
\cline { 2 - 2 } Poor liars & 9 & 0.18 & 0.39 \\
Neutral liars & 12 & 0.16 & 0.37 \\
Good liars & $\mathbf{1 8}$ & $\mathbf{0 . 2 6}$ & $\mathbf{0 . 4 4}$
\end{tabular}

\begin{tabular}{|c|c|c|c|c|}
\hline Miscellaneous strategies & 44 & 0.23 & 0.42 & $\chi^{2}(2)=1.29, p=.524, V=.082$ \\
\hline Poor liars & 9 & 0.18 & 0.39 & \\
\hline Neutral liars & 17 & 0.23 & 0.42 & \\
\hline Good liars & 18 & 0.26 & 0.44 & \\
\hline
\end{tabular}

\begin{tabular}{lcccc} 
No strategy & 10 & 0.05 & 0.22 & $\chi^{2}(2)=8.26, p=.016, V=.206$ \\
\cline { 2 - 3 } Poor liars & $\mathbf{6}$ & $\mathbf{0 . 1 2}$ & $\mathbf{0 . 3 3}$ & \\
Neutral liars & 4 & 0.05 & 0.23 & \\
Good liars & 0 & 0 & 0 & \\
& & & & \\
Not Applicable & 15 & 0.08 & 0.27 & $\chi^{2}(2)=1.23, p=.540, V=.080$ \\
Poor liars & 4 & 0.08 & 0.27 & \\
Neutral liars & 4 & 0.05 & 0.23 & \\
Good liars & $\mathbf{7}$ & $\mathbf{0 . 1 0}$ & $\mathbf{0 . 3 1}$ &
\end{tabular}

Note. The $N$ column represents the number of participants who endorsed each strategy, both in the total sample and for Poor, Neutral and Good liars, respectively. The total number of endorsed strategies surpasses the sample size of 194 because each participant could report multiple strategies that may have fallen into more than one category. Higher mean values indicate a more frequent endorsement of the respective strategy, whereas lower mean values indicate a less frequent endorsement of the respective strategy. The bolded numbers represent the group with the highest endorsement of each strategy.

Verbal and nonverbal strategies. To investigate whether participants differed in their endorsement of the importance of verbal versus nonverbal strategies based on their self-reported deception ability, we conducted two between-subjects ANOVAs with deception ability (Poor, Neutral, Good) on participants' Likert scale ratings of the general importance of verbal and nonverbal strategies. Additionally, the data were examined by calculating Bayesian ANOVAs with default prior scales, using JASP software. We report the Bayesian factors $(B F$; for interpretation, see Jarosz \& Wiley, 2014; Lee \& Wagenmakers, 2013) in line with the guidelines by Jarosz and Wiley (2014), adjusted from Jeffreys (1961). For ease of interpretation, $B F_{10}$ is used to indicate the Bayes factor as evidence in favour of the alternative hypothesis, whereas $B F_{01}$ is used to indicate the Bayes factor as evidence in favour of the null hypothesis.

First, we found a significant effect of self-reported deception ability on participants' endorsement of the importance of verbal strategies, $F(2,191)=5.62, p=.004, \eta_{\mathrm{P}}^{2}=.056 ; B F_{10}$ 
$=7.11$. Post hoc comparisons indicated that Good liars rated verbal strategies as significantly more important than Neutral liars $\left(M_{\text {diff }}=-0.82,95 \%\right.$ CI $\left.[-1.47,-0.18], p=.009\right)$, and Poor liars $\left(M_{\text {diff }}=-0.83,95 \%\right.$ CI $\left.[-1.54,-0.11], p=.018\right)$. Participants across deception ability groups did not differ with respect to their endorsement of the importance of nonverbal strategies, $F(2,191)$ $=.003, p=.997, \eta_{\mathrm{P}}^{2}<.001 ; B F_{01}=18.55$.

Next, we examined which specific verbal strategies participants reported to use when lying. We asked participants to indicate, from a list of ten options, which strategies they use. Table 2.2 provides an overview of the strategies endorsed by Poor, Neutral, and Good liars. Across all groups, the most frequently reported strategies were "Keeping the statement clear and simple" (endorsed by $17.6 \%$ of participants), “Telling a plausible story" (15.1\% of participants), "Using avoidance/being vague about details" (13.2\% of participants) and "Embedding the lie into an otherwise truthful story" (13.1\% of participants). To examine differences in the endorsement of each of the predetermined verbal strategies across Poor, Neutral, and Good liars we conducted a series of one-way between-subjects ANOVAs. Significant differences emerged for eight of the strategies, as follows: "Embedding the lie," $F(2,191)=11.97, p<.001, \eta_{\mathrm{P}}^{2}=$ $.111 ; B F_{10}=1438.20 ;$ "Matching the amount of details in the deceptive component of the statement to the truthful component, $" F(2,191)=4.77, p=.010, \eta_{\mathrm{P}}^{2}=.048 ; B F_{10}=3.32$; "Matching the type of details of the deceptive component of the statement to the truthful component, " $F(2,191)=3.56, p=.030, \eta_{\mathrm{P}}^{2}=.036 ; B F_{10}=1.15 ;$ "Keeping the statement clear and simple, $" F(2,191)=5.07, p=.007, \eta_{\mathrm{P}}^{2}=.050 ; B F_{10}=4.15 ;$ "Telling a plausible story, , $F(2,191)=5.48, p=.005, \eta_{\mathrm{P}}^{2}=.054 ; B F_{10}=5.98 ;$ "Providing unverifiable details, " $F(2,191)$ $=4.95, p=.008, \eta_{\mathrm{P}}^{2}=.049 ; B F_{10}=3.78$, and “Avoidance," $F(2,191)=3.79, p=.024, \eta_{\mathrm{P}}^{2}=$ $.038 ; B F_{10}=1.43$. Interestingly, Good liars reported using all of the above strategies significantly more than Poor liars (all $p$ 's $<.025$ ). The only exception was that Poor liars reported using the avoidance strategy significantly more than Good liars $(p=.026)$. Finally, there were no 
significant differences between Good, Neutral, and Poor liars in endorsing "Reporting from previous experience/memory" $\left(F(2,191)=1.32, p=.268, \eta \mathrm{P}^{2}=.014 ; B F_{01}=5.96\right)$, "Using complete fabrication" $\left(F(2,191)=0.57, p=.565, \eta_{\mathrm{P}}^{2}=.006 ; B F_{01}=11.36\right)$, and "Using other strategies" $\left(F(2,191)=0.51, p=.600, \eta_{\mathrm{P}}^{2}=.005 ; B F_{01}=11.96\right)$. See Table 2.2 for the exact values and applicable post hoc comparisons.

Table 2.2

Endorsement of predetermined deception strategies and descriptive statistics as a function of deception ability

\begin{tabular}{|c|c|c|c|c|c|}
\hline \multirow[t]{2}{*}{ Interview Strategies } & \multirow[t]{2}{*}{$N$} & \multirow[t]{2}{*}{$M$} & \multirow[t]{2}{*}{$S D$} & \multicolumn{2}{|c|}{ Bonferroni Comparisons } \\
\hline & & & & Poor & Neutral \\
\hline $\begin{array}{l}\text { Keeping the statement clear and } \\
\text { simple }\end{array}$ & 112 & & & & \\
\hline Poor liars & 20 & 0.39 & 0.49 & & \\
\hline Neutral liars & 49 & 0.65 & 0.48 & .010 & \\
\hline Good liars & 43 & 0.63 & 0.49 & .025 & 1.00 \\
\hline Telling a plausible story & 96 & & & & \\
\hline Poor liars & 17 & 0.33 & 0.48 & & \\
\hline Neutral liars & 36 & 0.48 & 0.50 & .302 & \\
\hline Good liars & 43 & 0.63 & 0.49 & .004 & .195 \\
\hline Avoidance & 84 & & & & \\
\hline Poor liars & 28 & 0.55 & 0.50 & & \\
\hline Neutral liars & 35 & 0.47 & 0.50 & 1.00 & \\
\hline Good liars & 21 & 0.31 & 0.47 & .026 & .167 \\
\hline Embedding the lie & 83 & & & & \\
\hline Poor liars & 13 & 0.26 & 0.44 & & \\
\hline Neutral liars & 26 & 0.35 & 0.48 & .850 & \\
\hline Good liars & 44 & 0.65 & 0.48 & $<.001$ & $<.001$ \\
\hline Providing unverifiable details & 76 & & & & \\
\hline Poor liars & 12 & 0.24 & 0.43 & & \\
\hline Neutral liars & 29 & 0.39 & 0.49 & .251 & \\
\hline Good liars & 35 & 0.52 & 0.50 & .006 & .338 \\
\hline $\begin{array}{l}\text { Matching the type of details } \\
\text { between lies and truths }\end{array}$ & 71 & & & & \\
\hline Poor liars & 12 & 0.24 & 0.43 & & \\
\hline Neutral liars & 27 & 0.36 & 0.48 & .453 & \\
\hline Good liars & 32 & 0.47 & 0.50 & .025 & .503 \\
\hline
\end{tabular}


Reporting from previous

experience

Poor liars

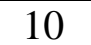

0.20

0.40

Neutral liars

$23 \quad 0.31$

0.46

Good liars

22

0.32

0.47

.535

.387

1.00

Matching the amount of details

between lies and truths

Poor liars

$\frac{38}{5}$

Neutral liars

12

0.10

0.30

Good liars

21

0.16

0.37

0.31

0.47

1.00

.012

.072

Using complete fabrication

Poor liars

14

Neutral liars

0.04

0.20

Good liars

0.08

0.27

0.09

0.29

1.00

.930

1.00

Using other strategies

Poor liars

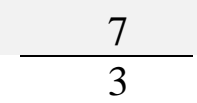

0.06

0.24

Neutral liars

2

0.03

0.16

Good liars

$\begin{array}{llll}2 & 0.03 & 0.17 & 1.00\end{array}$

$\begin{array}{llll}2 & 0.03 & 0.17 & 1.00\end{array}$

1.00

1.00

Note. The $N$ represents the number of participants who endorsed each strategy per group. Post hoc comparisons were conducted with the Bonferroni correction, and the $p$-values are displayed in the table. Higher mean values indicate a more frequent endorsement of the respective strategy, whereas lower mean values indicate a less frequent endorsement of the respective strategy. The bolded numbers represent the significant cell comparisons.

\section{Exploratory Testing of Liar Characteristics}

Finally, we also explored the associations between sex and education level and laypeople's self-reported deception ability by conducting a series of chi square tests of association. We observed a significant association between sex and deception ability, $\chi^{2}(2)=$ $12.31, p=.002, V=.253$. Further examination revealed that, of those who self-reported to be Poor liars, $70 \%(n=35)$ were female compared to $30 \%(n=15)$ male. Additionally, of those who identified themselves as Good liars, $62.7 \%(n=42)$ were male whereas $37.3 \%(n=25)$ were female. Both column proportions were significantly different at the .05 level. We did not observe a significant association between participants' education level and their self-reported deception ability, $\chi^{2}(4)=9.09, p=.059, V=.153$. The complete analyses are presented in Appendix 1 to conserve chapter length.

\section{Discussion}


We found that self-reported good liars (i) may be responsible for a disproportionate amount of lies in daily life, (ii) tend to tell inconsequential lies, mostly to colleagues and friends, and generally via face-to-face interactions, and (iii) highly rely on verbal strategies of deception, most commonly reporting to embed their lies into truthful information, and to keep the statement clear, simple and plausible.

\section{Lie Prevalence and Characteristics}

First, we replicated the finding that people lie, on average, once or twice per day, including its skewed distribution. Nearly $40 \%$ of all lies were reported by a few prolific liars. Furthermore, higher self-reported ratings of individuals' deception ability were positively correlated with self-reports of: (i) telling a greater number of lies per day, (ii) telling a higher frequency of white lies and exaggerations, (iii) telling the majority of lies to colleagues and friends or others such as romantic partners, and (iv) telling the majority of lies via face-to-face interactions. Importantly, skewed distributions were also observed for the other lie characteristics, suggesting that it may be misleading to draw conclusions from sample means, given that this does not reflect the lying behaviours of the average person. A noteworthy finding is that prolific liars also considered themselves to be good liars.

The finding that individuals who consider themselves good liars report mostly telling inconsequential lies is somewhat surprising. This deviates from the results of a previous study, which showed that prolific liars reported telling significantly more serious lies, as well as more inconsequential lies, compared to everyday liars (Serota \& Levine, 2015). However, small, white lies are generally more common (e.g., Feldman et al., 2002) and people who believe they can get away with such minor falsehoods may be more inclined to include them frequently in daily interactions. It is also possible that self-reported good liars in our sample had inflated perceptions of their own deception ability because they tell only trivial lies versus lies of serious consequence. 
Regarding the other lie characteristics, we found a positive correlation between selfreported deception ability and telling lies to colleagues, friends and others (e.g., romantic partners). This variation suggests that good liars are perhaps less restricted in who they lie to, relative to other liars who tell more lies to casual acquaintances and strangers than to family and friends (e.g., DePaulo \& Kashy, 1998). Our results also showed that good liars tended to prefer telling lies face-to-face. This fits the findings of one of the only other studies to examine characteristics of self-reported good versus poor liars, which found that self-perceived good liars most commonly lied via face-to-face interactions versus through text chat (Van Swol \& Paik, 2017). This could be a strategic decision to deceive someone to their face, since people may expect more deception via online environments (e.g., Whitty \& Carville, 2008). As researchers continue to examine the nature of lying and to search for ways of effectively detecting deception, it is important to recognise how certain lie characteristics may influence individuals' detectability as liars.

\section{Deception Strategies}

We also isolated the lie-telling strategies of self-reported good liars. People who identified as good liars placed a higher value on verbal strategies for successfully deceiving. Additional inspection of the verbal strategies reported by good liars showed that commonly reported strategies were embedding lies into truthful information and keeping their statements clear, simple and plausible. In fact, good liars were more likely than poor liars to endorse using these strategies, as well as matching the amount and type of details in their lies to the truthful part/s of their story, and providing unverifiable details. A common theme among these strategies is the relation to truthful information. This fits with the findings of previous literature, that liars typically aim to provide as much experienced information as possible, to the extent that they do not incriminate themselves (e.g., Leins et al., 2013; Nahari \& Vrij, 2015). Additionally, good liars used plausibility as a strategy for succeeding with their lies. This reflects the findings of the 
meta-analysis by Hartwig and Bond (2011) that implausibility is one of the most robust correlates of deception judgements, and the results of DePaulo et al. (2003) that one of the strongest cues to deception is liars' tendency to sound less plausible than truth-tellers $(d=-0.23)$.

We also found that self-reported poor liars were more likely than good liars to rely on the avoidance strategy (i.e., being intentionally vague or avoiding mentioning certain details). Previous research suggests that this is one of the most common strategies used by guilty suspects during investigative interviews (Granhag \& Hartwig, 2008). Additionally, all liars in our study expressed behavioural strategies as being important for deceiving successfully. This could be explained by the widespread misconceptions about the associations between lying and behaviour, for example that gaze aversion, increased movement or sweating are behaviours symptomatic of deception (e.g., Bogaard, Meijer, Vrij, \& Merckelbach, 2016; Vrij, 2008).

There was inconsistency in our data between the responses to the qualitative strategy question and the multiple-response strategy question. Based on the qualitative strategy data it seems that Good, Neutral, and Poor liars do not differ in their use of strategies. However, robust differences emerged when we evaluated participants' endorsement of the predetermined strategies. One explanation for this finding the difficulty people perceive when they have to verbalize the reasons for their behavior. Ericsson and Simon (1980) suggest that inconsistencies can occur especially when the question posed is too vague to elicit the appropriate information, which might have been the case in our study. Another explanation for the discrepancy in the data between the two measurement procedures is that data-driven coding is inherently susceptible to human subjectivity, error, and bias (Morrissey, 1974; Syed \& Nelson, 2015). Such limitations apply to a lesser extent to coding based on predetermined categories that are derived from psychological theory, an approach which has been heavily used within the deception literature (Vrij, 2008a). In any case, future research should continue exploring the deception strategies of good liars using a variety of methodological approaches. In particular, 
it would be beneficial to establish more reliable techniques for measuring interviewees' processing regarding their deception strategies. One potential idea could be to explore the effectiveness of using a series of cued questions to encourage the recall of specific aspects of interviewees' memory or cognitive processing. Another suggestion is to combine the datadriven and theory-driven approaches, whereby the coding system is generated inductively from the data but the coders draw from the theoretical literature when identifying categories (Syed \& Nelson, 2015).

\section{Limitations}

Some methodological considerations should be addressed. First, the results of the present study are drawn from participants' self-reports about their patterns of deception in daily life. Sources of error associated with such self-report data limit our ability to draw strong inferences from this study. However, previous research has validated the use of self-report to measure lying prevalence by correlating self-reported lying with other measures of dishonesty (Halevy et al., 2014). Moreover, self-report data may not be as untrustworthy as critics argue, and in some situations, it may be the most appropriate methodology (Chan, 2009). This study was intended as an initial examination of the strategies and preferences of good liars, and surveying liars for their own perspectives provided a novel source of insight into their behaviour. A constraint to the generalisability of this research is that we did not establish the ground truth as to whether self-reported good liars are indeed skilled deceivers. Future research could attempt to extend our findings by examining deceivers' lie frequency, characteristics, and strategies after systematically testing their lie-telling ability within a controlled laboratory setting.

Second, one of the most frequent concerns about using Amazon MTurk relates to low compensation and resulting motivation (e.g., Changler \& Shapiro, 2016; Landers \& Behrend, 2015). We took measures to ensure that our remuneration to participants was above the fair price for comparable experiments. Importantly, data collected through MTurk produces equivalent 
results as data collected from online and student samples (Casler, Nickel, \& Hackett, 2013; Feitosa, Joseph, \& Newman, 2015; Landers \& Behrend, 2015). As well, mTurk surveys have been shown to produce a representative sample of the United States population that yields results akin to those observed from more expensive survey techniques, such as telephone surveys (Fleischer, Mead, \& Huang, 2015). It speaks to the validity of our data, for example, that the selfreported prevalence of lies, and the endorsement of nonverbal deception strategies, replicates previous research. Nonetheless, the results of this study could be advanced if future research (i) directly replicates our survey amongst different populations, for instance university students, and (ii) conceptually replicates this research by evaluating different methodological approaches for measuring deception ability (e.g., via controlled evaluation) and good liars' strategies for deceiving (e.g., via cued recall).

\section{Implications and Future Research}

This study explored the deception characteristics and strategies used by self-reported good liars. Deception researchers generally agree that the most diagnostic information is found in the content of liars' speech (Bond \& DePaulo, 2006). Content-based cues to deception, however, may be less effective for detecting good liars who rely highly on verbal strategies of deception. This could even offer an explanation for the modest effect sizes observed in the deception literature (Luke, 2019). For instance, good liars in our study reported to strategically embed their lies into truthful information. This finding has potential implications for the reliability of credibility assessment tools that derive from the assumption that truth-tellers' statements are drawn from memory traces whereas liars' statements are fabricated from imagination (Johnson \& Raye, 1981; Steller \& Köhnken, 1989). If good liars draw on their memory of truthful previous experiences, then their statements may closely resemble those of their truth-telling counterparts. Another interesting observation was that self-reported good liars were more likely than poor liars to provide unverifiable details. This fits with the findings of 
previous literature on the VA, which contends that liars provide information that cannot be verified to balance their goals of being perceived as cooperative and of minimizing the chances of falsification by investigators (Nahari et al., 2014a, 2014b). A fruitful avenue of future research could be to further explore liars' strategic inclusion of truthful information and unverifiable details. Doing so may give lie detectors an advantage for unmasking skilled liars. It would also be interesting for future research to examine how good versus poor liars are affected by certain interview techniques designed to increase the difficulty of lying such as the reverse-order technique (Evans, Michael, Meissner, \& Brandon, 2013).

\section{Conclusion}

In sum, this study yields new insights into the deception prevalence, characteristics, and strategies used by self-reported good liars. We replicated the finding that a minority of individuals account for the majority of lies told in daily life, and we provide evidence that these prolific liars also consider themselves good liars. We unveiled several lie characteristics of good liars: They lean towards telling inconsequential lies, mostly to colleagues and friends, and generally via face-to-face interactions. Additionally, our results showed that self-reported good liars may attempt to strategically manipulate their verbal behaviour to stay close to the truth and to tell a plausible, simple, and clear story. This study provides a starting point for further research on the meta-cognitions and patterns of skilled liars who may be most likely to evade detection in investigative settings. 


\title{
Chapter 3: The Interaction of Truthful and
}

\author{
Deceptive Information
}

This chapter was published as:

Verigin, B. L., Meijer, E. H., Vrij, A., \& Zauzig, L. (2019). The interaction of truthful and deceptive information. Psychology, Crime and Law, 26, 367-383. doi: 10.1080/1068316X.2019.1669596 


\begin{abstract}
Research consistently shows that truthful accounts are richer in detail than deceptive accounts. It is unknown, however, how interviewees strategically regulate the information they provide when their accounts contain both truthful and deceptive information. This study examined how truths and lies interact, and whether interviewees' self-reported strategies reflect such interactions. Participants $(n=144)$ provided one statement consisting of two elements. We manipulated the veracity of these elements, with participants allocated to either both truthful, both deceptive, or one truthful and the other deceptive conditions. Results indicated that interviewees calibrate the richness of detail provided in the first element of their statement based on the veracity of the following element. Moreover, our exploratory tests revealed that lies become more detailed when they are flanked by truthful information relative to when they are flanked by other deceptive information. The finding that truthful and deceptive information interacts to influence detail richness provides insight into liars' strategic manipulation of information when statements contain a mixture of truths and lies. Strategic manipulations of this kind could potentially threaten the reliability of commonly used verbal lie detection tools. This study also offers insight to legal practitioners who rely on baseline deviations to assess credibility.
\end{abstract}




\section{Introduction}

In the legal arena, ascertaining the credibility of an interviewee remains an integral component of the investigative process. However, credibility assessment is a challenging task. Decades of research indicate that humans are poor lie detectors, rarely achieving accuracy rates above chance level (Bond \& DePaulo, 2006). One of the explanations for the poor lie detection performance is an overreliance on behavioural cues. No single behaviour, nor group of behaviours, is systematically and reliably indicative of deception (DePaulo et al., 2003; Hartwig \& Bond, 2011; Wright \& Wheatcroft, 2017), yet people continue to base their judgements on non-diagnostic behavioural cues such as gaze aversion or fidgeting (Bogaard, Meijer, Vrij, \& Merckelbach, 2016; Strömwall, Granhag, \& Hartwig, 2004).

Research regarding verbal deception detection is more promising. A stable finding within the literature is that liars' statements contain significantly fewer details than truth-tellers' statements (Amado, Arce, Fariña, \& Vilarino, 2016; DePaulo et al., 2003; Vrij, 2008a). More specifically, liars' statements contain less perceptual, spatial, and temporal details than truthtellers' statements (Vrij, 2008a, 2008b). Much of these findings stem from research on CriteriaBased Content Analysis (CBCA; Steller \& Köhnken, 1989) and Reality Monitoring (RM; Alonso-Quecuty, 1992, 1995; Johnson, Bush, \& Mitchell, 1998; Johnson \& Raye, 1981). Both CBCA and RM assume that recollections of personally experienced events are more detailed and coherent than statements about unexperienced or fabricated events (Johnson \& Raye, 1981; Undeutsch, 1967, 1989; Vrij, 2005, 2008a) because memories of external origin (i.e., truthful reports) are based on perceptual processes whereas memories of internal origin (i.e., deceptive reports) are based on individuals' imagination and reasoning. In particular, it can be reasoned that truths represent experienced memories and are more likely to include perceptual, contextual and affective information and to be more clear and plausible than lies, which are based on imagination (Johnson \& Raye, 1981). 
Statements often consist of a mixture of both truths and lies. When given the opportunity, liars will incorporate their deception into descriptions of previous experiences, so called embedded lies. Evidence for the prevalence of embedded lies comes from Leins, Fisher, and Ross (2013). In two studies, these authors found that the majority of liars - 67\% in the first study and $86 \%$ in the second - chose to formulate their deceptive account based on a previously experienced event. Additionally, Nahari et al. (2014) found that of their 44 mock criminals who were instructed to provide deceptive statements, more than half indicated that over $20 \%$ of their statement was truthful. The use of embedded lies has been observed across various populations, whether it is research participants (Bell \& DePaulo, 1996; Leins, Zimmerman, \& Polander, 2017), non-criminals engaging in deception (DePaulo et al., 2003), or criminal suspects (Hartwig, Granhag, \& Strömwall, 2007).

The embedding of lies into otherwise truthful statements is also reflected in the findings from research examining the strategies interviewees adopt to appear credible (Clemens, Granhag, \& Strömwall, 2013; Fiske \& Taylor, 2008). For example, Hartwig and colleagues' (2007) examination of guilty and innocent suspects' strategies revealed that one of liars' most endorsed strategies was to avoid lying by telling the truth as much as possible. Interviewees' strategic attempts to be perceived as credible can be explained by the theory of self-regulation, a framework for understanding how people are motivated to control their behaviour to move away from undesired outcomes and to reach desired goals (Carver \& Scheier, 2012; Hartwig, Granhag, \& Luke, 2014). Liars strategically attempt to edit reality to create a plausible, logical story (Granhag, Strömwall, \& Jonsson, 2003; Vrij, Granhag, \& Mann, 2010) that may have its foundations in truthful previous experiences. In contrast, innocent interviewees are generally forthcoming and aim to provide full, candid accounts (Hartwig et al., 2007) using their memory to reconstruct what happened. 
In the present study, we investigated to what extent truthful and deceptive information interacts to influence detail richness. The rationale for expecting truths and lies to interact is based on research into beliefs about cues to deception. Several studies have shown that people believe inconsistencies are a sign of deception (Blair, Reimer, \& Levine, 2018; Vredeveldt, van Koppen, \& Granhag, 2014). Consequently, it is not surprising that liars report to try to maintain consistency as a strategy to appear credible. In general, liars tend to be most concerned with reducing the amount of inconsistencies within the details of their statement compared to any other type of inconsistency (e.g., between-statement or statement-evidence inconsistencies; Deeb et al., 2017, 2018). As a result of their efforts to maintain consistency, liars tend to be equally or more consistent than truth-tellers (Granhag \& Strömwall, 2002; Granhag et al., 2003; Vredeveldt et al., 2014), who - as a normal function of memory - may appear inconsistent as information is naturally added or forgotten (e.g., Fisher, Brewer, \& Mitchel, 2009).

In one of the only studies to include statements that purposefully contained both truths and lies, Deeb et al. (2017) instructed liars to provide a statement containing reports of a deceptive event and a truthful event during two interviews. When asked about their strategies for appearing credible, nearly half of the liars (45\%) reported an attempt to maintain consistency across the interviews for both events. Moreover, many liars reported that they did so by strategically lowering their "baseline consistency" by including fewer repetitions in non-critical portions of the interview. In contrast, only $8 \%$ of truth-tellers reported using the consistency strategy (Deeb et al., 2017). Given liars' focus on consistency, it is plausible that when their statements contain both truthful and deceptive information their efforts to maintain consistency may extend to the richness of information provided.

In sum, lies are rarely complete fabrications. Yet, this is often how they are treated in research (Vrij, 2008a), leaving a significant gap within the deception literature. Examination of statements consisting of both truths and lies could account for individual differences in deceiving 
(see Vrij, 2016) and provide insight into liars' verbal behaviour. As such, the objective of the current experiment was twofold. First, we extended the findings of Deeb et al. (2017) to examine how truths and lies interact to influence the consistency of detail richness across elements of a statement. Second, we examined to what extent such interactions are reflected in deceivers' selfreported strategies. To examine this, the participants in our study provided a statement consisting of two elements. We manipulated the veracity of these elements, with participants either delivering both truthful, both deceptive, or one truthful and the other deceptive element. Based on the general verbal deception literature, we predicted that truthful elements would be richer in detail than deceptive elements (Hypothesis 1). There are two ways in which participants could maintain consistency. First, by calibrating the content of the second element of their statement to that of the first element. This would imply that elements preceded by a lie would be less detailed than elements preceded by a truth (Hypothesis 2). Second, it is also possible that participants would anticipate the second element, and calibrate the content of the first element to that of the second. Our next prediction was therefore that elements followed by a lie would be less detailed than those followed by a truth (Hypothesis 3). Finally, we expected that the interaction between lies and truths would be, at least in part, reflected in the participants' self-reported strategies (Hypothesis 4).

\section{Method}

\section{Participants}

The sample consisted of 144 native-German speaking undergraduate students (116 females; 28 males) who participated in exchange for either course credit or a $€ 7.50$ voucher. Participants described their ethnicity as Caucasian $(n=116)$, Asian $(n=5)$, Hispanic $(n=2)$, or 'Other' $(n=21)$. A-priori power analysis suggested that to achieve an $85 \%$ likelihood of detecting a true difference given a medium effect size $(f=.25$; Cohen, 1988), 146 participants were required. To allow for an equal distribution across conditions we opted for 144 participants. All 
participants were between 18 and 26 years old $(M=20.81, S D=1.70)$, and had not yet received any information on lie detection or interviewing techniques in their curriculum. The study was approved by the standing ethical committee.

\section{Design}

The present study used a 2 (Veracity of the first element [truth, lie]) by 2 (Veracity of the second element [truth, lie]) between-subjects factorial design. Our primary dependent measure was the richness of detail (i.e., quantity of perceptual, spatial, and temporal information combined) in each of the elements separately.

\section{Procedure}

Upon arriving to the lab and providing informed consent, participants completed a demographic questionnaire measuring their age, sex, race, native language and education. Afterwards, they received a sealed envelope that contained a letter instructing them to complete no task, one task, or two tasks. The envelope was labelled only by participant number to ensure the researcher was blind to conditions during the interview. Task A consisted of helping to develop a promotional flyer for a café located at the University campus. Participants were instructed to walk across campus to the café and to use the camera provided to take photos that could be included on a flyer to promote the café. In Task B, participants were requested to walk across campus to the bus stop located at the University Medical Centre. Upon arrival, they had to look for a woman named Michelle, of whom they were provided with a photo and informed she would be arriving by bus at some time that day. They were asked to wait for a minimum of five minutes, and to use the notepad and pen provided to write down the information of any buses that arrived or departed during their time. Participants were told to take a photo of Michelle using the camera provided, if they saw her arrive. In reality, Michelle was a fictitious character and participants did not encounter her during the task. Participants were given up to thirty-five 
minutes to complete their task/s. Both tasks were designed to ensure comparable duration and difficulty, as well as similarities regarding participants' familiarity with the routes and locations.

Participants were randomly assigned to complete no task, one task, or two tasks. For the participants who completed two tasks, the order was counter-balanced. Upon returning to the laboratory after completing their assigned task/s, participants received a second sealed envelope explaining they would be interviewed by the researcher about Tasks A and B and that they were to report and answer questions as if they had completed both tasks. As a result, we created four (between-subject) veracity conditions: Lie-Lie (participants who completed neither of the tasks), Lie-Truth and Truth-Lie (participants who completed either Task A or Task B), and Truth-Truth (participants who completed both tasks). The instruction letter contained a brief description of what Tasks A and B entailed to allow those who did not complete one or both of the tasks to familiarise themselves with what they would be reporting (see Appendix 2). This also allowed liars to know, in advance of providing their statement, during which element/s of their statement they were required to lie. Participants were told it was important to be convincing because (i) it would prevent them from having to stay an additional twenty minutes to provide a written account and (ii) it would earn them a chance to win a $€ 50$ raffle prize. After receiving these instructions, participants were given ten minutes alone to prepare.

Next, participants underwent a structured, information-gathering style interview (see Appendix 2). The interviews were audio recorded. At the outset of the interview, the researcher stated that her goal was to obtain as much information as possible, and to determine the participant's credibility. The researcher also reminded participants that she was blind to the veracity condition, and instructed them to report as many details as possible, even if they did not think they were important. Each interview began with the elicitation of a free narrative of the participants' activities during the first task. The researcher then asked a series of questions such as "What else can you tell me about this task?", "How long did this task take you?", "Did 
anything unexpected happen?" The same procedure was repeated for the second task. Afterwards, the researcher gave participants an opportunity to provide any missing information regarding either task.

Following the interview, participants completed the Post-Interview Questionnaire. They were asked to rate several items on 5-point Likert scales (1 - strongly agree to 5 - strongly disagree): (i) I felt motivated to convince the interviewer that I completed both tasks, (ii) I had enough time to prepare for the interview, (iii) I prepared my statements strategically ${ }^{3}$, (iv) I was successful in convincing the interviewer that I completed both tasks, (v) I think I will have to stay longer to provide a written statement. Participants then responded to two open-ended questions regarding their strategies for convincing the interviewer of their credibility and their strategic preparation. As well, participants responded to two multiple-response questions that asked them to select the verbal strategies (e.g., forthcomingness, avoidance, telling a plausible or clear statement, providing unverifiable details; see Table 3.2) and nonverbal strategies (e.g., maintaining eye contact, not fidgeting, appearing calm) they used when reporting each of the tasks separately. After completing the questionnaire, participants were debriefed and the experiment was concluded. None of the participants were asked to stay longer to provide a written statement and all participants were included in the raffle. The experiment lasted approximately one hour.

Coding. Verbal content analysis. The audio recorded interviews were transcribed verbatim. The Principal Investigator, who has expertise with verbal statement analysis, extensively trained two independent coders, both of whom were blind to the veracity of the statements. These coders assessed the statements for detail richness, represented by the sum of three types of detail; specifically, (1) perceptual details: Information about what the interviewee saw (e.g., "She wore a red blouse"), heard (e.g., "He talked loudly"), smelled (e.g., "It $\underline{\text { smelled }}$

\footnotetext{
${ }^{3}$ One participant's response to this question was not recorded via Qualtrics and therefore the results to this specific question are based on $N=143$.
} 


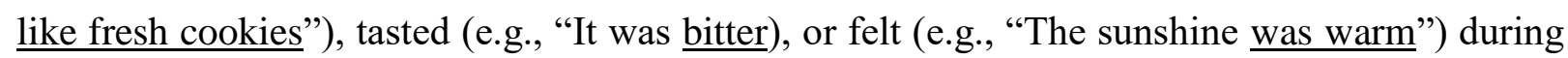
their activities; (2) spatial information: Information about locations (e.g., “On the elevator”) or the spatial arrangement of people and/or objects (e.g., "The cups were on the bar"); and (3) temporal information: Information about when the activities or event happened (e.g., "It was

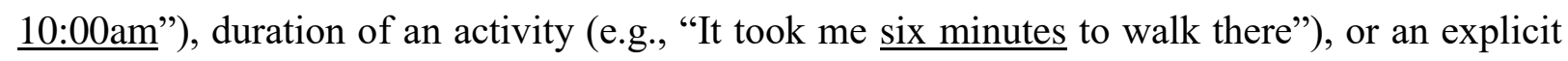
description of a sequence of events (e.g., "After getting my coffee, I left"). For each participant, the coders marked all perceptual, spatial, and temporal details individually in the text, and tallied the frequency of occurrence of each of these details for the element of the statement dealing with Task A and Task B individually. This coding scheme is publically available on the Open Science Framework.

The main coder and the second coder coded a randomly selected $20 \%$ of the statements in order to establish reliability. Inter-rater reliability between the two coders for each of the criteria in the total statements, using the two-way random effects model measuring consistency (e.g., Vrij, Leal, Jupe, \& Harvey, 2018; Koo \& Li, 2016), was high for perceptual information (Single Measures, Intraclass correlation coefficient, ICC $=.91$ ), spatial information $(I C C=.89)$, and temporal information $(I C C=.75)$. After confirming the reliability between the two coders, the second coder rated an additional 17 statements, and the main coder completed the remaining sample of participants' statements.

For our analyses, we calculated a total richness of detail score by summing the number of perceptual, spatial, and temporal details for the elements of the statement relating to Task A and Task B separately.

Finally, we also coded for the clarity of statements (i.e., relating to RM; Johnson \& Raye, 1981; Vrij, 2008a) ${ }^{4}$. This criterion was scored as present (1) if the statement was clear, sharp and vivid and scored as absent (0) if the statement was vague and dim. As well, we coded for the

\footnotetext{
${ }^{4}$ Inter-rater reliability between the two coders for the clarity of the total statements, using the two-way random effects model measuring consistency, was excellent (Single Measures, ICC $=.91$ ).
} 
plausibility of statements and for the presence of unexpected complications. The latter two variables are not reported in the chapter because we observed floor effects for each variable and therefore cannot report reliable data.

Strategies. To code the participants' self-reported strategies, one main coder examined the open-ended responses to establish data-driven categories (see Masip \& Herrero, 2013). This entailed a multi-stage process that began by identifying each participant's strategy or strategies, then grouping together overlapping responses, and gradually condensing these responses into key categories based on conceptual similarities. The Principal Investigator oversaw each stage of this process and decided upon the final categories. A total of eight categories emerged from this coding method (see Table 3.1).

The main coder and a second coder then coded a randomly selected $20 \%$ of the participants' open-ended responses in order to establish inter-rater reliability regarding the classification of responses into the appropriate categories. Both coders were extensively trained in qualitative content analysis by the Principal Investigator. After confirming that the raters were consistent (Single Measures ICCs ranging from .55 to 1.00$)^{5}$, the main coder completed the remaining sample of participant responses and only these scores were used in the analysis.

In addition to coding participants' interview strategies, we also examined their selfreported methods of interview preparation. The same qualitative coding method as above was used for preparation coding. Inter-rater reliability was excellent (Single Measures ICCs ranging from 95 to 1.00). The main coder's scores for the entire sample were used in the analyses.

\section{Analysis}

To test whether the participants calibrated the richness of details of the elements according to the veracity of the preceding or following element, we conducted two $2 \times 2$ ANOVAs. First, we tested the effect of the first element on the second by submitting the richness

\footnotetext{
${ }^{5}$ The average $I C C$ across the eight strategies categories was .80. Two categories, relating to general linguistic control and behavioural control had low to moderate inter-rater reliability.
} 
of details in the second element to a 2 (veracity of the second element: [truth, lie]) X 2 (veracity of the first element: [truth, lie]) between-subjects ANOVA. Second, we tested the effect of the second element on the first element by submitting the richness of details in the first element to a 2 (veracity of the first element: [truth, lie]) X 2 (veracity of the second element: [truth, lie]) between-subjects ANOVA. Additionally, the data were examined by calculating a Bayesian ANOVA with default prior scales (i.e., $r$ scale fixed effects at 0.5), using JASP. We report the Bayesian factors (BF; e.g., Lee \& Wagenmakers, 2013) in line with the guidelines by Jarosz and Wiley (2014), adjusted from Jeffreys (1961). The approximate evidence categories are as follows: Positive values between 1 and 3 indicate weak evidence for the alternate or null hypothesis, values between 3 and 10 indicate substantial evidence, values between 10 and 20 constitutes strong or very strong evidence, and values above 20 are considered very strong or decisive evidence. Evidence for the interaction term was calculated by dividing the interaction model by the main factors (e.g., Wagenmakers et al., 2016). For ease of interpretation, $B F_{10}$ is used to indicate the Bayes factor as evidence in favor of the alternative hypothesis, whereas $B F_{01}$ is used to indicate the Bayes factor as evidence in favor of the null hypothesis.

\section{Results}

\section{Motivation, Preparation, \& Self-Perceived Success}

Participants reported to have been highly motivated and to have had enough time to prepare for the interview, with no significant differences between veracity groups. Significant differences emerged between conditions in terms of strategic preparation and self-perceived success. Participants reported more strategic preparation for deceptive elements of the statements than for truthful elements. Additionally, participants who first reported deceptive elements were more likely than any other group to believe that they would have to stay longer to provide a written statement. To conserve manuscript length, the exact analyses are reported in Appendix 2. 


\section{Interview Preparation Techniques}

Across veracity conditions, four preparation techniques emerged from our qualitative coding of participants' responses. Participants in the Lie-Lie condition reported the highest overall frequency of preparation techniques whereas those in the Truth-Truth condition reported the lowest. The respective endorsement of the four techniques was similar across veracity conditions, with the most frequently endorsed technique being to "Use imagination to prepare the statement", followed by "Strategically preparing the statement and/or responses", "Purposefully manipulating the content of the statement", and "Other or miscellaneous". The exact analyses are reported in Appendix 2.

\section{Statement Clarity}

Veracity significantly influenced the perceived clarity of both the first and second element of interviewees' statements only when they were reporting entirely truthfully or entirely deceptively, with truthful statements being evaluated as more clear than deceptive statements. The complete analyses are presented in Appendix 2.

\section{Richness of Detail}

Confirmatory analyses. To test whether elements preceded by a lie would be less detailed than elements preceded by a truth (Hypothesis 2) we conducted a 2 (veracity of the second element: [truth, lie]) X 2 (veracity of the first element: [truth, lie]) between-subjects ANOVA on the richness of details in the second element. This analysis revealed a main effect of veracity of the second element, $F(1,140)=10.98, p=.001, \eta_{\mathrm{P}}^{2}=.073 ; B F_{10}=22.00$, with truthful elements $(M=34.76, S D=18.26,95 \%$ CI [31.00, 38.53] $)$ scoring higher on richness of details than deceptive elements $(M=25.85, S D=14.11,95 \% \mathrm{CI}[22.09,29.61])$. The main effect of the veracity of the first element was not significant, $F(1,140)=3.01, p=.085, \eta_{\mathrm{P}}^{2}=.021 ; B F_{01}=$ 1.57 , meaning that the elements preceded by a lie $(M=27.97, S D=17.07,95 \% \mathrm{CI}[24.21,31.73])$ were not significantly less rich in detail than elements preceded by a truth $(M=32.64, S D=$ 
$16.45,95 \%$ CI $[28.88,36.40])$. Finally, the interaction effect was also not significant, $F(1,140)$ $=2.00, p=.160, \eta_{\mathrm{P}}^{2}=.014 ; B F_{01}=1.74$, indicating the veracity of the first element had no differential effect on the richness of detail score of the second element. Taken together, we received support for Hypothesis 1, that truthful elements are richer in detail than deceptive elements; however, we did not show support for our second hypothesis, that interviewees would calibrate the content of the second element to that of the first. See Figure 3.1.

\section{Richness of Detail within Element Two}

Preceded by: $\square$ Truth $\square$ Lie

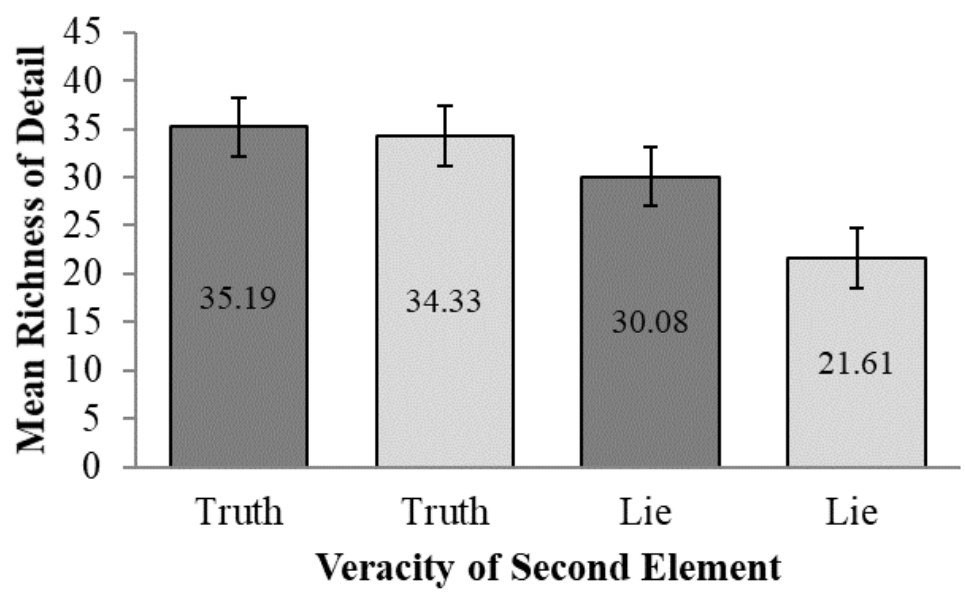

Figure 3.1. Mean richness of details in element two as a function of veracity condition. Standard errors are represented by the error bars attached to each symbol.

To investigate whether participants calibrated the first element according to the second element (Hypothesis 3) we conducted a 2 X 2 (veracity of the first element: [truth, lie] X veracity of the second element: [truth, lie]) between-subjects ANOVA on the richness of details in the first element. The main effect of veracity of the first element was significant, $F(1,140)=9.45, p$ $=.003, \eta \mathrm{P}^{2}=.063 ; B F_{10}=10.79$, with truthful elements $(M=35.71, S D=14.42,95 \%$ CI $[32.75$, 38.67]) being richer in detail than deceptive elements $(M=29.19, S D=11.27,95 \%$ CI [26.23, 32.16]). The main effect of veracity of the second element was also significant, $F(1,140)=5.60$, $p=.019, \eta_{\mathrm{P}}^{2}=.038 ; B F_{10}=1.95$, with the elements followed by a lie $(M=29.94, S D=13.48$, 
$95 \%$ CI $[26.98,32.91])$ scoring lower on richness of details than the elements followed by a truth $(M=34.96, S D=12.73,95 \% \mathrm{CI}[32.00,37.92])$. Lastly, the interaction effect was not statistically significant, $F(1,140)=1.50, p=.222, \eta_{\mathrm{P}}^{2}=.011 ; B F_{01}=2.12$, indicating the veracity of the second element had no differential effect on the richness of detail score of the first element. Overall, we found additional support for Hypothesis 1, that truthful elements are richer in detail than deceptive elements, and we supported our third hypothesis, that interviewees would calibrate the content of the first element to that of the second. See Figure 3.2.

\section{Richness of Detail within Element One}

Followed by: $\square$ Truth $\square$ Lie

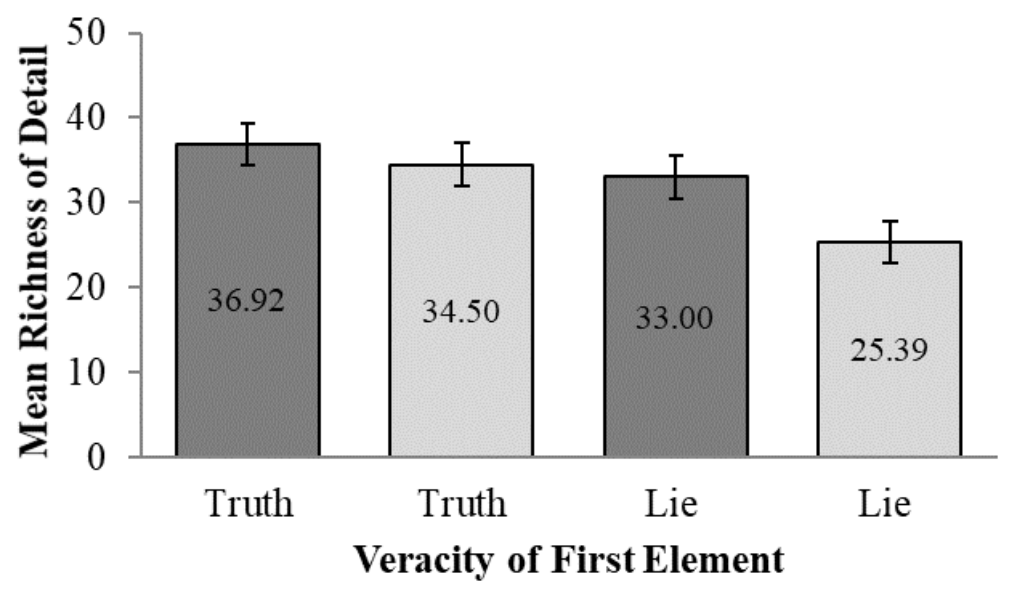

Figure 3.2. Mean richness of details in element one as a function of veracity condition. Standard errors are represented by the error bars attached to each symbol.

Exploratory analyses. As we were specifically interested in how lies are influenced by truthful information, we also carried out two exploratory independent-samples $t$-tests comparing the richness of details of lies only. We corrected for inflated type 1 error probability by applying a Bonferroni correction dividing the alpha of .05 by two, resulting in a significance level of .025 . We found a statistically significant difference in mean richness of detail score of the deceptive second element between those preceded by a truth versus by a lie, $t(70)=-2.66, p=.010 ; B F_{10}$ $=4.64$, with those preceded by a lie $(M=21.61, S D=10.45,95 \%$ CI $[14.80,28.42])$ being 
significantly less rich in detail than those preceded by a truth $(M=30.08, S D=16.05,95 \% \mathrm{CI}$ [23.27, 36.89]). There was also a statistically significant difference in mean richness of detail score between deceptive first elements followed by a truth versus by a lie, $t(66.34)=-3.03, p=$ $.004 ; B F_{10}=10.81$, indicating that a lie followed by another lie $(M=25.39, S D=9.34,95 \% \mathrm{CI}$ $[21.20,29.58])$ was significantly less detailed than a lie followed by a truth $(M=33.00, S D=$ $11.86,95 \%$ CI $[28.81,37.19])$.

\section{Interviewees' Strategies}

The vast majority of interviewees reported using strategies to enhance the likelihood that they would be perceived as credible: $95.49 \%$ of participants reported using one or more verbal strategies (e.g., telling a plausible story, keeping the statement clear and simple, reporting from previous memory; see Table 3.2 ) and $92.02 \%$ indicated using at least one nonverbal strategy (e.g., maintaining eye contact, not fidgeting, appearing confident, etcetera) during their interview. In this section, we will concentrate our reports primarily on the verbal strategies relating to our consistency hypotheses.

Overall interview strategies. Table 3.1 provides an overview of the data derived from the coding of participants' open-ended responses regarding their overall interview strategies. Across all veracity conditions, the most frequently mentioned verbal interview strategy was to "Provide a detailed and plausible account" (36.68\% of all reported strategies). Only 7.34\% of the reports fell into the category of "Manipulating the verbal content by maintaining consistency between the statement elements" (e.g., matching the type and quantity of details provided in both elements, adapting the deceptive story to the truthful story or vice versa, etcetera). As shown in Table 3.1, only participants in the Truth-Lie and Lie-Truth conditions reported strategies relating to maintaining consistency between their statements, with no significant difference in mean scores for endorsing the consistency strategy between these two conditions, $t(70)=0.70, p=$ $.486 ; B F_{01}=3.33$. Thus, participants in the mixed veracity conditions reported similarly (albeit 
infrequent) strategic attempts to match the consistency of their reports, whereas participants who fully lied or fully told the truth, did not report to use such a strategy.

Table 3.1

Frequency and percentage of overall interview strategies across veracity conditions Condition

Interview Strategy $\quad$ Truth-Truth Lie-Lie Truth-Lie Lie-Truth

$\begin{array}{lcccc}\text { Provide a detailed, plausible account } & 21 & 22 & 27 & 25 \\ & \mathbf{( 4 0 . 3 8 )} & \mathbf{( 3 0 . 9 9 )} & \mathbf{( 3 8 . 0 3 )} & \mathbf{( 3 8 . 4 6 )} \\ \text { Strategic control of behaviour } & 5 & 18 & 12 & 9 \\ & (9.62) & (\mathbf{2 5 . 3 5 )} & \mathbf{( 1 6 . 9 0 )} & \mathbf{( 1 3 . 8 5 )} \\ \text { Manipulate verbal content: } & 1 & & & \\ \text { General linguistic control } & (1.92) & (12.68) & (11.27) & (10.77) \\ \text { Manipulate verbal content: } & 5 & 11 & 2 & 5 \\ \text { Include truthful details } & (9.62) & (15.49) & (2.82) & (7.69) \\ \text { Use imagination to deliver the statement } & 2 & 5 & 5 & 9 \\ & (3.85) & (7.04) & (7.04) & (13.85) \\ \text { Manipulate verbal content: } & 0 & 0 & 11 & 8 \\ \text { Maintain consistency between elements } & (0) & (0) & (15.49) & (12.31) \\ & & & & \\ \text { No strategy } & 7 & 6 & 4 & 1 \\ & (13.46) & (8.45) & (5.63) & (1.54) \\ \text { Provide a truthful account } & 11 & 0 & 2 & 1 \\ \quad \text { Total frequency count per condition } & 52 & 71 & 71 & 65\end{array}$

Note. The numbers reported represent the frequency occurrence of each strategy. The respective percentage within each condition is presented in brackets. Frequencies may add up to over 36 (the number of participants in each condition) because each participant could report multiple strategies that may have fallen into one or more categories. The bolded numbers represent the categories with the two largest percentages per veracity condition.

Interview strategies for individual statement elements. We were also interested in interviewees' strategies, relative to each element of the statement individually. We asked participants to indicate which strategies they used for their reports of each Task A and Task B 
separately from a predetermined response set of multiple verbal strategies (see Table 3.2). Regarding the strategies for the second element of their statements, one of the most frequently endorsed strategies by participants in the Truth-Lie condition was "Matching the amount of details in statements" (13.82\% of the endorsed strategies among this condition). Similarly, the same matching strategy was the most frequently endorsed strategy by participants in the LieTruth condition (17.89\% of the endorsed strategies among this condition). This provides partial support for our prediction that interviewees in the mixed veracity conditions would report having strategically calibrated their verbal content based on the veracity of the preceding element. Taken together, these findings contribute partial support to Hypothesis 4, which predicted that the interaction between lies and truths would be, at least in part, reflected in participants' selfreported strategies.

Table 3.2

Frequency of verbal strategy endorsement for element one and two across conditions

\begin{tabular}{lccccc}
\hline \multicolumn{1}{c}{ Interview Strategy } & \multicolumn{3}{c}{ Condition } & & \\
& $\begin{array}{c}\text { Truth- } \\
\text { Truth }\end{array}$ & $\begin{array}{c}\text { Lie- } \\
\text { Lie }\end{array}$ & $\begin{array}{c}\text { Truth- } \\
\text { Lie }\end{array}$ & $\begin{array}{c}\text { Lie- } \\
\text { Truth }\end{array}$ & Total \\
\hline Strategies for Element One & 30 & 8 & 29 & 11 & 78 \\
Forthcomingness & 13 & 24 & 12 & 23 & 72 \\
Telling a plausible story & 10 & 21 & 15 & 25 & 71 \\
Providing details the investigator cannot check & 17 & 21 & 11 & 15 & 64 \\
Keeping the statement clear and simple & 6 & 22 & 7 & 19 & 54 \\
Reporting from previous experience/ memory & 1 & 11 & 2 & 18 & 32 \\
Outright fabrication & 3 & 12 & 5 & 10 & 30 \\
Avoidance & 2 & 0 & 0 & 1 & 3 \\
Other & 1 & 0 & 0 & 0 & 1 \\
None & 83 & 119 & 81 & 122 & 405 \\
Total frequency counts & & & & & \\
Strategies for Element Two & 8 & 26 & 29 & 12 & 75 \\
Telling a plausible story & 10 & 21 & 21 & 15 & 67 \\
Providing details the investigator cannot check & 13 & 22 & 13 & 18 & 66 \\
Keeping the statement clear and simple & 28 & 7 & 9 & 21 & 65 \\
Forthcomingness & 9 & 13 & 21 & 22 & 65 \\
Matching the amount of details in statements & 8 & 12 & 19 & 16 & 55 \\
Matching the type of details in statements & & & &
\end{tabular}




\begin{tabular}{lccccc} 
Reporting from previous experience/ memory & 8 & 12 & 19 & 8 & 47 \\
Outright fabrications & 5 & 9 & 13 & 1 & 28 \\
Avoidance & 2 & 14 & 8 & 7 & 31 \\
Other & 1 & 1 & 0 & 1 & 3 \\
None & 2 & 1 & 0 & 2 & 5 \\
Total frequency counts & 94 & 138 & 152 & 123 & 507 \\
\hline
\end{tabular}

Note. The numbers reported represent the frequency of participants who endorsed each strategy.

\section{Discussion}

In line with previous research (e.g., Amado et al., 2016; DePaulo et al., 2003; Vrij, 2008a), we found support for our hypothesis that truthful elements are richer in detail than deceptive elements (Hypothesis 1). We also found evidence that truthful and deceptive information interacts to influence detail richness: (i) Elements followed by a lie were less detailed than elements followed by a truth (Hypothesis 3), and (ii) deceptive elements became more detailed when flanked by a truth than when flanked by a lie.

Participants only calibrated the detail richness of the first element based on the veracity of the second element. An explanation for this could be that participants knew, prior to the interview, whether they would be deceptive or honest about each element of the statement. When participants anticipated having to tell a lie in the second element of their statement, they may have already focused their efforts on this from the beginning of their interview (Jundi, Vrij, Hope, Mann, \& Hillman, 2013). The directed attention of their cognitive resources towards ensuring the latter element of the statement was perceived as credible may have impaired the detail richness of the former element of the statement. This directed attention could even provide an alternative explanation for the differences in detail richness between lies and truths. Given that these lies are most likely to actually consist of a mixture of truths and lies, it may not be memory processes, but directed attention that can account for the typical finding that lies are less detailed than truths. 
We did not find support for our prediction that elements preceded by a lie would be less detailed than elements preceded by a truth (Hypothesis 2). Yet, our exploratory tests of lies only did reveal that participants calibrated their lies according to both the preceding and the following element, with deceptive elements becoming more detailed when flanked by a truth than when flanked by a lie. The discrepancy between these two findings may mean we had insufficient power to detect the main effect and/or interaction in the omnibus test. This is supported by the Bayes factors of 1.57 and 1.74, supplying only weak evidence for the absence of such effects. However, the results of the exploratory tests suggest that liars were intentionally calibrating the detail richness of their lies to that of the truths, perhaps to avoid noticeable inconsistencies between the truthful and deceptive elements of the statement. Future studies could examine whether this effect replicates, and if so, how lies become richer in detail.

From a motivational perspective, participants in the mixed veracity conditions had the same task: Providing a statement with one truthful element and one deceptive element. As a strategy, they could either (i) boost the richness of details within the deceptive element making it resemble the truthful part, or (ii) reduce the detail richness within the truthful element to make it resemble the deceptive element. Our exploratory findings - that participants in the Truth-Lie and Lie-Truth conditions provided more details than participants in the Lie-Lie condition suggest that interviewees applied the first strategy, increasing the detail richness of the deceptive element to match that of the truthful element. More broadly, the order of presenting the truths and lies within statements, and not solely the veracity, could have influenced the richness of details provided by participants in the mixed veracity conditions. Specifically, interviewees may have preferred to begin by telling the truth and to integrate their lie midway through the statement, a pattern previously observed in a study examining deception within an insurance claim setting (Leal, Vrij, Nahari, \& Mann, 2016). The tendency for insurance claimants to begin by reporting truthfully and to tell their lies as the interview progressed may have been an attempt 
to gain the investigator's trust or to become more comfortable with the interview setting and investigator. There may also have been a cognitive reason: Beginning with a lie increases cognitive demand meaning that interviewees have to formulate and intentionally activate a plausible lie while suppressing the truth (e.g., Vrij, 2015), during an unfamiliar situation. Future research should continue teasing apart the motivational and cognitive processes of liars who report both truthful and deceptive information within one statement.

We found only limited support for our prediction that the differences in the richness of details in statements would be reflected in participants' strategies (Hypothesis 4). Participants in the mixed veracity conditions reported similar, albeit infrequent, attempts to match the consistency of their statements, and participants who fully lied or fully told the truth, did not utilise such a strategy. The relatively low number of participants reporting to have used a consistency strategy corresponds to the modest effect sizes found in our quantitative analyses. As argued by Ericsson and Simon (1980), when asking participants to make retrospective judgements regarding their behaviour, inconsistencies can arise because of the experimental procedures, particularly when using questions that are too general to prompt the information actually sought. Since we asked broad strategy questions, this could have led to less accurate responses.

Our research was not without limitations. First, we examined the effect of two elements immediately following each other. This is appropriate to establish whether an effect appears, but future research could utilise a less artificial paradigm that better translates to applied contexts; for example, examining statements with lies and truths dispersed throughout. Similarly, our results may not generalise to situations in which the liar is unable to anticipate the exact topic or direction of the interview, such as when unexpected questions are asked. Perhaps in such situations, participants' strategic attempts to maintain consistency would not calibrate predominantly in the direction of the following element. Third, we cannot conclude that 
participants, who were instructed to lie, provided lies that were entirely untruthful. In fact, in the current study, participants across veracity conditions reported to have strategically included truthful details they had drawn from previous experiences and/or memory. Hence, we are left with deceptive statements that may be, realistically, a combination of truths and lies, which may have weakened the strength of the observed effects. Indeed, participants in the mixed veracity conditions may have easily borrowed truthful details from their experience of the completed task for their descriptions of the fabricated task, whereas complete liars may not have experienced any event rich in detail during the allotted time. This strengthens the argument that liars in the mixed veracity conditions draw on recent truthful previous memories to calibrate their statements whereas liars who provide entirely deceptive accounts may not. Fourth, the focus of this study was on one particular aspect of statement consistency: Consistency in detail richness. It is also possible that the elements of participants' statements were consistent, or inconsistent, on other dimensions than detail richness, such as linguistic characteristics.

Another consideration is that the emotional pressure experienced by liars during actual investigative interviews is conceivably much higher than during psychological experiments. Additionally, nearly 80 percent of our sample was female, a disproportion that may also impact the generalisability of our findings since the majority of perpetrators that come to the attention of the criminal justice system are male (e.g., Heimer \& Lauritsen, 2008; U.S. Department of Justice, 2009). However, it is unlikely that stakes or gender robustly influenced our results since the same theoretical assumptions and strategies should apply across low and high stakes contexts and for males and females.

We observed floor effects for the variables regarding unexpected complications and plausibility. One potential explanation is that the presence of complications may be situationrelated. The low rate of reported complications in our sample could be because the reports were about short encounters scripted by the researcher, as opposed to longer activities initiated by the 
participant (e.g., Vrij et al., 2018). Additionally, the statements may have been judged as implausible since they were about unique experimental tasks as opposed to more believable dayto-day activities.

The research presented in this chapter has two practical implications. First, our results show that liars are able to calibrate the detail richness of their lies to that of their truths. This presents a possible threat to the diagnostic accuracy and utility of verbal credibility assessment tools if liars are able to provide lies that mirror the richness of detail in the truthful components of their statement (e.g., Gnisci et al., 2010; Leins et al., 2017; Nahari, Vrij, \& Fisher, 2012).

A second practical implication relates to the baseline technique. Baselining refers to the practice in which interviewers evaluate the veracity of a critical component of a statement relative to a baseline, or neutral, component of the same statement (see Vrij, 2016 for an overview). Baselining is frequently used by police in practice (Ewens, Vrij, Jang, \& Jo, 2014; Frank, Yarbrough, \& Ekman, 2006; Inbau, Reid, Buckley, \& Jayne, 2013). This approach encourages starting an interview with a neutral - and often truthful - part. Our findings indicate that interviewees calibrate the detail richness of the initial component of their statement based on the veracity of the following component. Therefore, if suspects manipulate the richness of details provided in their initial baseline statement to be consistent with the detail richness provided in their subsequent reports, then potential truth-lie differences may disappear. A possible preventative measure is for lie detectors to control for the event and to ask about the same event multiple times in different formats (e.g., first obtaining an oral account and then a sketch), using the first statement as a baseline (Vrij, 2016).

In sum, the current study addressed if and how truthful and deceptive information interacts to influence the richness of details in statements, and how this is reflected in individuals' strategies. The results indicate that interviewees calibrate the richness of detail provided in the first element of their statement based on the veracity of the following element, however, this 
effect was not robustly reflected in interviewees' self-reported strategies. Moreover, it seems that participants calibrate their lies according to both the preceding and the following element, with lies becoming more detailed when flanked by truthful information. 


\section{Chapter 4: Embedding Lies into Truthful Stories}

This chapter was published as:

Verigin, B. L., Meijer, E. H., \& Vrij, A. (2020). Embedding lies into truthful stories does not affect their quality. Applied Cognitive Psychology, 34, 516-525. doi: 10.1002/acp.3642 


\begin{abstract}
When given the opportunity, liars will embed their lies into otherwise truthful statements. In what way this embedding affects the quality of lies, however, remains largely unknown. This study investigated whether lies that are embedded into truthful stories are richer in detail and contain higher quality details compared to lies that are part of entirely fabricated statements. Participants $(N=111)$ were asked to provide a statement that was either entirely truthful, entirely fabricated, or had the fabricated element of interest embedded into an otherwise truthful story. Results indicated that lies embedded in a fabricated statement are not qualitatively different from lies embedded in an otherwise truthful statement. Supporting Bayes factors provided moderate to strong evidence for this conclusion. Accordingly, verbal credibility assessment tools based on the verbal content measured in this study may be robust against the embedding of lies.
\end{abstract}




\section{Introduction}

Whereas people typically report to rely on non-verbal behaviour to detect deception (Vrij, 2008), the accuracy of credibility assessments actually improves when judges rely only on the verbal content of a statement (Bond \& DePaulo, 2006). Moreover, good lie detectors report a higher reliance on verbal cues when making credibility judgments, whilst poor lie detectors tend to rely primarily on non-verbal cues (Mann, Vrij, \& Bull, 2004). Specifically, the most consistent finding in the verbal deception literature is that truthful statements contain more details than deceptive ones (e.g., Amado, Arce, Fariña, \& Vilarino, 2016; DePaulo et al., 2003; Luke, 2019; Oberlader et al., 2016). A recent meta-analysis estimated this effect at $d=$ 0.55 (Amado et al., 2016), while additional meta-analytical findings support the usefulness of temporal, visual, and auditory details for differentiating truthful from false accounts (Masip, Sporer, Garrido, \& Herrero, 2005).

In studies investigating the effect of deception on the content of statements, participants are typically instructed to report a truthful account, or to fabricate one. Real life deceptive statements will, however, rarely be complete fabrications, as liars prefer to embed their lies into otherwise truthful statements (e.g., Leins, Fisher, \& Ross, 2013; Nahari, 2018a; Nahari \& Vrij, 2015; Vrij, 2008; Vrij, Granhag, \& Porter, 2010). Yet, it remains unknown how embedded lies are influenced by surrounding truthful information. Reason to believe that the embedding of lies affects their quality stems from research on beliefs about cues to deception and liars' strategies. This research suggests that laypeople and legal professionals alike believe that inconsistency is symptomatic of deception (Blair, Reimer, \& Levine, 2018; Vredeveldt, van Koppen, \& Granhag, 2014; Strömwall \& Granhag, 2003). Accordingly, one of the main concerns of liars - and one of their most frequently reported strategies - is to maintain consistency (Deeb, Vrij, Hope, Mann, Granhag, \& Lancaster, 2017; Hartwig, Granhag, Strömwall, \& Doering, 2010). Specifically, Deeb et al. (2017) instructed liars to provide a 
statement containing a mix of a deceptive event and a truthful event. When asked about their strategies for appearing credible, $45 \%$ of liars mentioned maintaining consistency, and many liars reported to have maintained consistency by strategically lowering their "baseline consistency" by including fewer repetitions in specific portions of the interview.

If participants would successfully maintain consistency when embedding their lies into an otherwise truthful statement, their lies could become more richly detailed when surrounded by truthful information. Indeed, recent research compared how interviewees strategically regulate the information they provide when their accounts contain one truthful and one deceptive component, and found that lies became more richly detailed when preceded or followed by truthful information compared to when preceded or followed by other lies (Chapter 3 of this thesis). The present study aimed to extend these findings by examining the extent to which the embedding of lies into otherwise truthful stories affects the quality of information. In line with previous research (e.g., Amado et al., 2016; DePaulo et al., 2003; Masip et al., 2005; Luke, 2019), we predicted that completely truthful statements would be richer in detail compared to completely fabricated statements (Hypothesis 1). Based on our consistency assumption, we also predicted that lies embedded into an otherwise truthful statement would be richer in details than lies embedded into an entirely fabricated statement (Hypothesis 2).

Besides the differences in detail richness, we also investigated whether the consistency hypothesis extends to a number of other content cues that have been shown diagnostic of deception (see Table 4.1). As secondary cues, we first examined the amount of verifiable information. This cue stems from the Verifiability Approach (VA; Nahari, Vrij, \& Fisher, 2014a, 2014b), which works on the assumption that liars, on the one hand, are inclined to provide detailed statements to be perceived as cooperative and credible, but, on the other hand, want to minimise the chances that investigators can falsify their statement (Masip \& Herrero, 2013; Nahari et al., 2014a). A strategy that meets these aims is to provide information that 
cannot be verified. Second, we examined liars' tendency to report fewer complications occurrences that make a situation more difficult than necessary, often characterised by disrupted activity or failing efforts (e.g., missing the bus; Steller \& Köhnken, 1989; Vrij, Leal, Jupe, \& Harvey, 2018) - compared to truth-tellers. This makes sense given liars' preference for simple stories (Hartwig, Granhag, \& Strömwall, 2007). We also investigated common knowledge details (i.e., strongly invoked stereotypical information about events; Vrij, Leal, Mann et al., 2017). Whereas truth-tellers have personal, unique experiences of an event (DePaulo, Kashy, Kirkendol, Wyer, \& Epstein, 1996), liars typically lack such information and their reports tend to be characterised by more general, impersonal knowledge (Sporer, 2016). Another cue relates to self-handicapping strategies (i.e., implicit or explicit justifications as to why someone is unable to provide certain information; Vrij, Leal, Mann et al., 2017). Although liars prefer to keep their stories simple, they also realise that admitting a lack of knowledge and/or memory may generate suspicion from investigators (Ruby \& Brigham, 1998). Liars, then, are inclined to provide justifications for their inability to provide certain information.

Lastly, we explored the clarity and plausibility of statements. Clarity, or vividness, refers to the extent to which a statement is clear, sharp, and vivid (instead of dim and vague; e.g., Sporer, 2004). How plausible a statement is refers to whether the story is probable, realistic and makes sense. Both criteria derive from the Reality Monitoring method of credibility assessment (RM; Johnson \& Raye, 1981), which reasons that truthful accounts represent experienced memories and are likely to be, for example, more clear and plausible compared to lies, which are formed from imagination. There is previous empirical support regarding these critera (DePaulo et al., 2003; Leal, Vrij, Warmelink, Vernham, \& Fisher, 2015; Sporer and Küpper, 1995; Zhou, Burgoon, Nunamaker, \& Twitchnell, 2004).

Altogether, our secondary hypotheses were that completely truthful statements would be characterised by more verifiable details, a higher proportion of complications, and higher 
ratings of statement clarity and plausibility compared to completely fabricated statements (Hypothesis 3), and that lies embedded into an otherwise truthful statement would be characterised by these higher quality details moreso than lies embedded into an entirely fabricated statement (Hypothesis 4; see Table 4.1).

Table 4.1

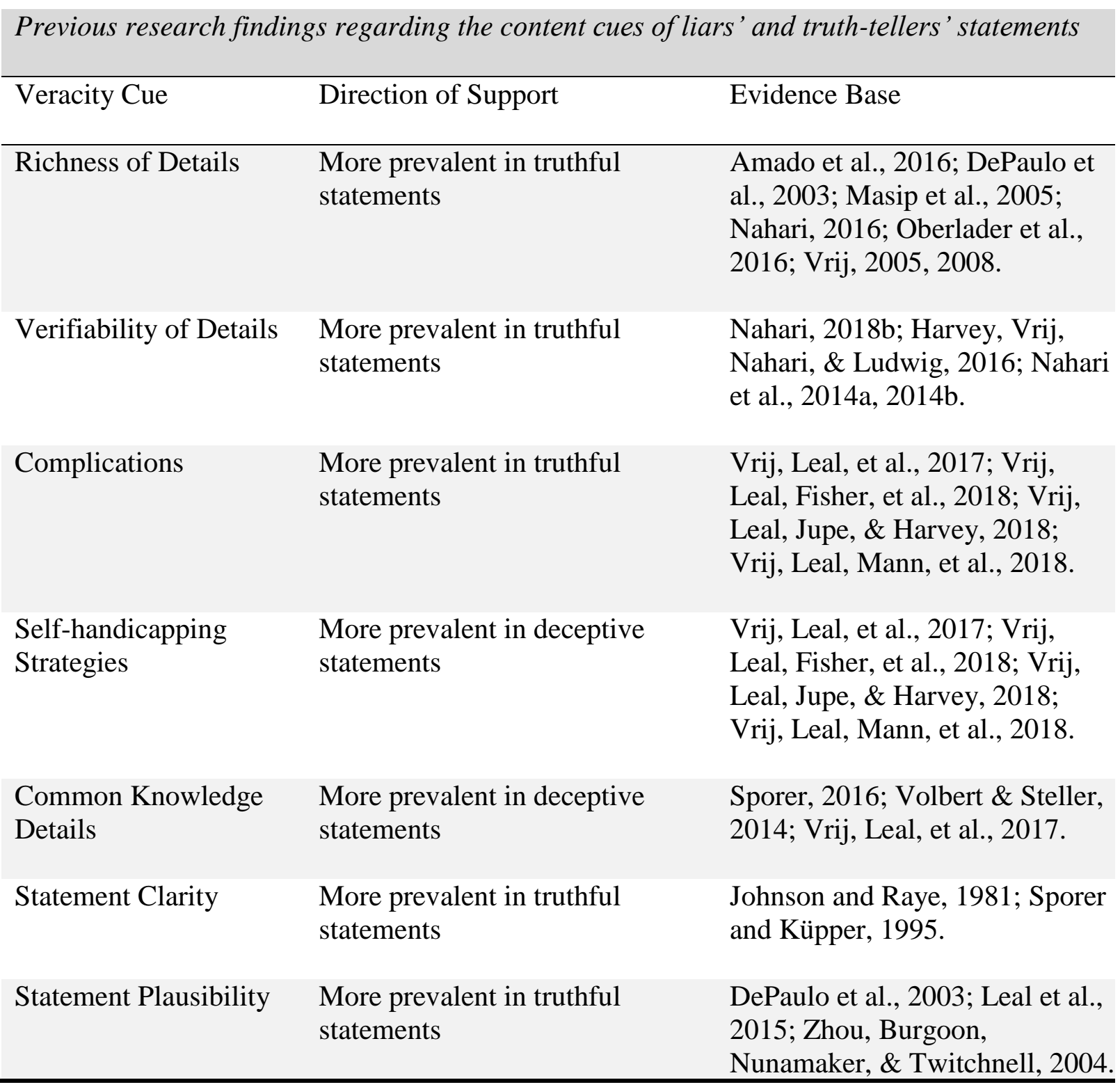

\section{Method}

\section{Participants}

The sample consisted of 111 undergraduate students, graduate students and staff members who were all naïve to forensic psychology (92 females; 19 males; $M_{\text {age }}=21.91$ years; $S D_{\text {age }}=$ 
4.25 years). Participants described their ethnicity as Caucasian $(n=63)$, Black $(n=6)$, Asian $(n$ $=6)$, or 'Other' $(n=36)$. An a-prior power analysis indicated that this number of participants was required to achieve an $80 \%$ likelihood of detecting a true difference given a medium effect size. All subjects participated in exchange for course credit or a $£ 5$ voucher and the opportunity to win a $£ 50$ raffle prize. Eligible participants were native-English speakers, aged 18 years or older. The study was approved by the standing ethical committee, and was pre-registered and approved via the Open Science Framework (OSF): http://j.mp/2D60QWu.

\section{Procedure}

Upon arriving to the lab and providing informed consent, participants completed a PreInterview Questionnaire followed by a demographic form measuring their age, sex, ethnicity, education and native-language. Participants then received a letter instructing them to imagine that they had been called into a police interview as a suspect in a burglary investigation, and that they must provide an alibi for their whereabouts during the day of the crime. Three conditions were created by providing participants with additional instructions. First, truth-tellers were told that they were innocent, and their task was to convince the interviewer of their innocence by providing a completely truthful alibi. In addition, two lie conditions were created. Liars were told to imagine they were guilty of the hypothetical crime in question, and that they must lie about their whereabouts during the time of the burglary, that took place between 1:00pm and 3:00pm. Embedded liars were instructed to embed the critical 1:00pm to 3:00pm period in an otherwise truthful account, whereas in the Complete lie condition, liars were asked to fabricate the entire account (see Appendix 3 for the instructions). All participants were told it was important to be convincing because it would earn them a chance to win a $£ 50$ voucher and it would prevent them from having to stay an additional twenty minutes to provide a written account. After receiving these instructions, participants were given up to ten minutes alone to prepare. 
The assignment to either the Truthful, Embedded lie, or Complete lie condition was done in a pseudo-random manner. The first five of every fifteen participants were assigned to the Truth-Teller condition, whereas the remaining participants (e.g., participants 6 to 15,21 to 30 , etcetera) were assigned to either the Embedded lie or Complete lie conditions. This was done so we could match the content of the critical period in the two lie conditions to that in the truthful condition. Specifically, the alibi activity that participants were instructed to lie about was generated based on the truth-tellers' responses to the Pre-Interview Questionnaire that asked them to briefly describe, in approximately one sentence, their activities between 1:00pm to 3:00pm on the previous three days. The Principal Investigator selected the activity that had the most unique, contextual detail and this selected activity was used for all three conditions, while making sure the assigned alibi activity differed from any of the liars' reported events. This pseudo-randomised design allowed us to experimentally control the type of activity reported and length of time between the experience and reporting (i.e., one, two or three days) across participants, thereby reducing heterogeneity across statements.

Next, a second researcher (blind to participants' conditions) began the interview by stating that her goal was to obtain as much information as possible, and to determine how credible the participant's account was. The interviewer instructed the participant to report as many details as possible, even if s/he did not think they were important. Each interview followed a structured format (see Appendix 3) and was video-recorded. The interview began with the elicitation of a free narrative of the participants' activities from morning to evening on the day in question. The researcher then asked several questions, such as "What else can you tell me about that day?", "Did anything unexpected happen or perhaps something that did not go as planned?" Interviewees were also asked to report their activities during the 1:00pm to 3:00pm period specifically, and were given the opportunity to provide any forgotten or missing information at the end of the interview. 
Following the interview, participants were informed that the experimental portion of the study had ended, that their answers to the following questionnaire would not influence how their statement would be assessed, and that they should answer the next questions honestly. Participants then completed the Post-Interview Questionnaire, where they were asked to rate several items on 5-point Likert scales (1 - strongly agree to 5 - strongly disagree): (i) The instructions clearly explained what I needed to do, (ii) I had enough time to prepare for the interview, (iii) I was motivated to convince the interviewer that I was innocent, (iv) I was successful in convincing the interviewer that I was innocent, (v) I prepared my statements strategically, (vi) The interviewer was friendly. Next, participants evaluated the truthfulness of both the critical and general components of their alibi using a 10-point scale (1-not at all truthful to 10 - completely truthful). Finally, participants were debriefed and the experiment was concluded. None of the participants were asked to stay longer and all participants were included in the raffle. Participation in the study took approximately one hour.

Coding. Statements were assessed for the presence of spatial information (e.g., "Sitting in the row behind my friend"), temporal information (e.g., "It was $\underline{6: 00 \mathrm{pm}}$ ") and perceptual information (e.g., "I saw him sitting at the bar"; richness of detail), the verifiability of detail (e.g., a receipt of purchase), and the presence of complications (e.g., missing the bus), common knowledge details (e.g., "We went to pick up groceries at the store") and self-handicapping strategies (e.g., "I can't tell you anything else because my friend did all of the planning"), and the clarity and plausibility of the statement (statement quality). The exact description of the verbal content coding can be found in Appendix 3. Two scores were created for each dependent measure; the first was the sum of all occurrences in the entire statement, the second constituted the sum within the time period from 1:00pm to $3: 00 \mathrm{pm}$. To establish reliability, the main coder and a second trained coder evaluated a randomly selected $20 \%$ of the statements. Using the twoway random effects model measuring consistency (see Koo \& Li, 2016), inter-rater reliability 
was high for spatial information (Single Measures, Intraclass correlation coefficient, $I C C=.79$ ), temporal information $(I C C=.93)$, and perceptual information $(I C C=.78)$. The combined richness of detail variable thus had excellent reliability $(I C C=.97)$, as did the verifiable details variable $(I C C=.91)$. The $I C C$ 's were high for statement clarity $(.90)$ and adequate for statement plausibility (.51), leading to a high reliability score for the combined statement quality variable $(I C C=.87)$. The reliability for coding the presence of complications, common knowledge details and self-handicapping strategies was also adequate $(I C C$ 's $=.60, .51$, and .71 , respectively). After confirming the reliability between the two coders, the main coder completed the remaining sample of participants' statements. In the analyses, we used only the scores of the main coder.

\section{Deviations from Preregistration}

The analyses reported here deviate from the preregistration in several ways. All deviations were decided upon prior to analysing the data. First, we preregistered two separate analyses, one based on 'quantity of details' (e.g., particular information regarding places, times, persons, objects and events) and one based on 'the richness of detail'. Instead, we limit our analysis to 'richness of detail,' a combination of all spatial, temporal and perceptual information. Second, we preregistered predictions based on a measure combining the frequency of complications, self-handicapping strategies, and common-knowledge details. Instead, we coded the frequency of each cue separately and calculated the proportion of complications score (complications/ [complications + common knowledge details + self-handicapping strategies]). This is in line with previous literature (see, for example, Vrij, Leal, Jupe et al., 2018) and has theoretical advantages given that it is a within-subjects comparison that is also sensitive to the different verbal strategies used by liars and truth-tellers. Finally, we specified a priori hypotheses regarding participants' self-reported strategies. We report these analyses in Appendix 3 to keep the manuscript within reasonable length.

\section{Results}




\section{Motivation, Preparation, and Self-Perceived Success}

No significant differences between the three Veracity conditions appeared for motivation, the clarity of instructions, preparation time, strategic preparation, and interviewer friendliness (see Appendix 3 for exact analyses). Significant differences between the Veracity conditions appeared for reported success in convincing the interviewer that they were innocent, $F(2,108)=$ $4.74, p=.011, \eta_{\mathrm{P}}^{2}=.081$. Truth-tellers reported being the most successful $(M=4.14, S D=0.82)$, followed by liars in the Embedded lie condition $(M=3.78, S D=0.82)$, and lastly liars in the Complete lie condition $(M=3.51, S D=0.96)$. Post hoc comparisons using the Bonferroni procedure indicated that the mean difference in self-perceived success was only statistically significant between the truth-tellers and liars in the Complete lie condition $(p=.008)$.

\section{Truthfulness Measures}

We asked participants to rate, on a scale of one to ten (one being not at all truthful and ten being completely truthful) how truthful the $1: 00 \mathrm{pm}$ to $3: 00 \mathrm{pm}$ component of their alibi statement was. Significant differences emerged between the conditions, $F(2,108)=136.21, p<$ $.001, \eta_{\mathrm{P}}^{2}=.716$. Truth-tellers reported that the critical component of their alibi was almost completely truthful $(M=9.59, S D=0.90)$, whereas liars in the Complete lie $(M=2.46, S D=$ 2.57) and Embedded lie $(M=2.62, S D=2.48)$ conditions indicated that only a small portion of their critical alibi component was truthful. Post hoc comparisons showed that the mean difference in reported truthfulness was statistically significant only between the truth-tellers and liars in the Complete lie condition $(p<.001)$ and between the truth-tellers and liars in the Embedded lie condition $(p<.001)$. Thus, the self-reported truthfulness of the critical component of interviewees' alibi statements conformed to the instructions they received across conditions.

Similarly, we asked participants to rate, on the same ten-point scale, how truthful their general alibi statement was, excluding the period from 1:00pm to 3:00pm. Significant differences emerged between the conditions, $F(2,108)=75.82, p<.001, \eta_{\mathrm{P}}^{2}=.584$. Truth-tellers reported 
that the majority of their general alibi was truthful $(M=9.32, S D=0.88)$. Liars in the Complete lie condition reported that a portion of their general alibi was truthful $(M=3.32, S D=2.76)$, whereas liars in the Embedded lie condition reported that the majority of their general alibi was truthful $(M=7.92, S D=2.45)$. Post hoc comparisons indicated that the mean difference in reported truthfulness was significant between truth-tellers and liars in the Embedded lie condition $(p=.002)$, between truth-tellers and liars in the Complete lie condition $(p<.001)$ and between liars in the Embedded lie and liars in the Complete lie conditions $(p<.001)$. As above, the selfreported truthfulness of the general component of interviewees' alibi statements mostly corresponded to the instructions they received across conditions.

\section{Statement Characteristics}

Our primary analyses focused on examining the characteristics between entirely truthful statements versus entirely fabricated statements, and between the deceptive 1:00pm to 3:00pm period embedded in lies and embedded in truths. Additionally, we conducted two exploratory analyses $^{6}$. We compared the characteristics of the deceptive 1:00pm to 3:00pm period embedded in truths with the truthful 1:00pm to $3: 00 \mathrm{pm}$ period also embedded into truths, and compared the truthful parts of the statement flanking the deceptive 1:00pm to 3:00pm period to the truthful parts of the statement flanking the truthful $1: 00 \mathrm{pm}$ to $3: 00 \mathrm{pm}$ period.

Confirmatory hypothesis testing. Statement characteristics were analysed using a series of univariate between-subjects ANOVAs. Additionally, the data were examined by calculating a Bayesian ANOVA with default prior scales (i.e., $\mathrm{r}$ scale fixed effects at 0.5), using JASP software. We report the Bayesian factors (BF; see Jarosz \& Wiley, 2014; Lee \& Wagenmakers, 2013) in line with the guidelines by Jarosz and Wiley (2014), adjusted from Jeffreys (1961). The

\footnotetext{
${ }^{6}$ An alternative analysis would be to run within-participant analyses to compare truth-lie differences. Such analyses are methodologically inappropriate as these comparisons potentially confound truth-lie differences with duration and activity; specifically, the critical period spanned two hours, whereas a morning and evening can describe a more variable period, and will likely describe different activities.
} 
approximate evidence categories are as follows: Positive values between 1 and 3 indicate weak evidence for the alternate or null hypothesis, between 3 and 10 indicate substantial evidence, between 10 and 20 constitutes strong or very strong evidence, and scores above 20 are considered very strong or decisive evidence. For ease of interpretation, $B F_{10}$ indicates the Bayes factor as evidence in favour of the alternative hypothesis, whereas $B F_{01}$ indicates the Bayes factor as evidence in favour of the null hypothesis.

Richness of detail. We first compared the completely truthful to the completely fabricated statements on the richness of details (i.e., amount of spatial, temporal, and perceptual information combined) by conducting a univariate between-subjects ANOVA. As predicted, truthful statements $(M=56.46, S D=27.85)$ scored higher on richness of details than fabricated statements $(M=41.38, S D=15.66), F(1,72)=8.24, p=.005, \eta_{\mathrm{P}}^{2}=.103, B F_{10}=7.51$, lending support to Hypothesis 1.

Next, we tested Hypothesis 2 by conducting a second univariate between-subjects ANOVA to compare the detail richness of the 1:00pm to 3:00pm period between the Embedded lie and Complete lie conditions. We did not observe a significant effect for the richness of detail, $F(1,72)=.21, p=.648, \eta_{\mathrm{P}}^{2}=.003, B F_{01}=3.80$, meaning that lies embedded into otherwise truthful statements were not significantly richer in detail than lies embedded into entirely fabricated statements. As such, we did not find support for Hypothesis 2.

Secondary content cues. To evaluate Hypothesis 3, we conducted a series of univariate between-subjects ANOVAs to compare the completely truthful to the completely fabricated statements on (i) the amount of verifiable details, (ii) the number of complications, common knowledge details and self-handicapping strategies, (iii) the total proportion of complications (i.e., complications/[complications + common knowledge details + self-handicapping strategies]), and iv) the quality of statements (i.e., the clarity/plausibility). Truthful statements $(M=.30, S D=.62)$ contained significantly fewer self-handicapping strategies than fabricated 
statements $(M=.65, S D=.86), F(1,72)=4.09, p=.047, \eta_{\mathrm{P}}^{2}=.054$, but the $B F_{10}$ of 1.34 was not very diagnostic. We did not observe significant effects of Veracity on the remaining dependent variables: The amount of verifiable details, $F(1,72)=1.76, p=.189, \eta_{\mathrm{P}}^{2}=.024$, $B F_{01}=1.96 ;$ the number of complications, $F(1,72)=1.54 p=.219, \eta_{\mathrm{P}}^{2}=.021, B F_{01}=2.15$; the number of common knowledge details, $F(1,72)=2.88, p=.094, \eta_{\mathrm{P}}^{2}=.038, B F_{01}=1.22$; the proportion of complications, $F(1,72)=2.65, p=.108, \eta_{\mathrm{P}}^{2}=.036, B F_{01}=1.34$; the quality of statements, $F(1,72)=.70, p=.407, \eta_{\mathrm{P}}^{2}=.010, B F_{01}=3.08$. The exact values can be found in Table 4.2. Hence, we received only partial support for Hypothesis 3, that completely truthful statements would contain more high quality content cues compared to fabricated accounts.

Table 4.2

\begin{tabular}{|c|c|c|c|c|}
\hline \multicolumn{5}{|c|}{$\begin{array}{l}\text { Exact values of dependent measur } \\
\text { a function of veracity condition }\end{array}$} \\
\hline Dependent & Veracity & Statement Component & Mean & Confidence Intervals \\
\hline Variable & Condition & & Value & $(95 \% \mathrm{CI})$ \\
\hline \multirow{7}{*}{$\begin{array}{l}\text { Richness of } \\
\text { Detail*+ }\end{array}$} & Truth-teller*+ & 1:00-3:00pm* & 21.22 & {$[17.10,25.33]$} \\
\hline & & & & \\
\hline & & General statement+ & 56.46 & {$[47.17,65.75]$} \\
\hline & Embedded liar* & $1: 00-3: 00 \mathrm{pm}^{*}$ & 15.22 & {$[12.00,18.43]$} \\
\hline & & General statement & 51.68 & {$[43.68,59.67]$} \\
\hline & Complete liar+ & $1: 00-3: 00 \mathrm{pm}$ & 14.24 & {$[11.39,17.09]$} \\
\hline & & General statement+ & 41.38 & {$[36.16,46.60]$} \\
\hline \multirow{7}{*}{$\begin{array}{l}\text { Verifiability of } \\
\text { Details }\end{array}$} & Truth-teller & $1: 00-3: 00 \mathrm{pm}$ & 3.14 & {$[2.29,3.98]$} \\
\hline & & & & \\
\hline & & General statement & 9.57 & {$[7.75,11.38]$} \\
\hline & Embedded liar & $1: 00-3: 00 \mathrm{pm}$ & 2.14 & {$[1.51,2.76]$} \\
\hline & & General statement & 8.14 & {$[6.68,9.60]$} \\
\hline & Complete liar & $1: 00-3: 00 \mathrm{pm}$ & 2.70 & {$[1.89,3.52]$} \\
\hline & & General statement & 8.00 & {$[6.43,9.57]$} \\
\hline $\begin{array}{l}\text { Statement } \\
\text { Quality* }\end{array}$ & Truth-teller* & $1: 00-3: 00 \mathrm{pm}^{*}$ & 2.95 & {$[2.58,3.31]$} \\
\hline
\end{tabular}




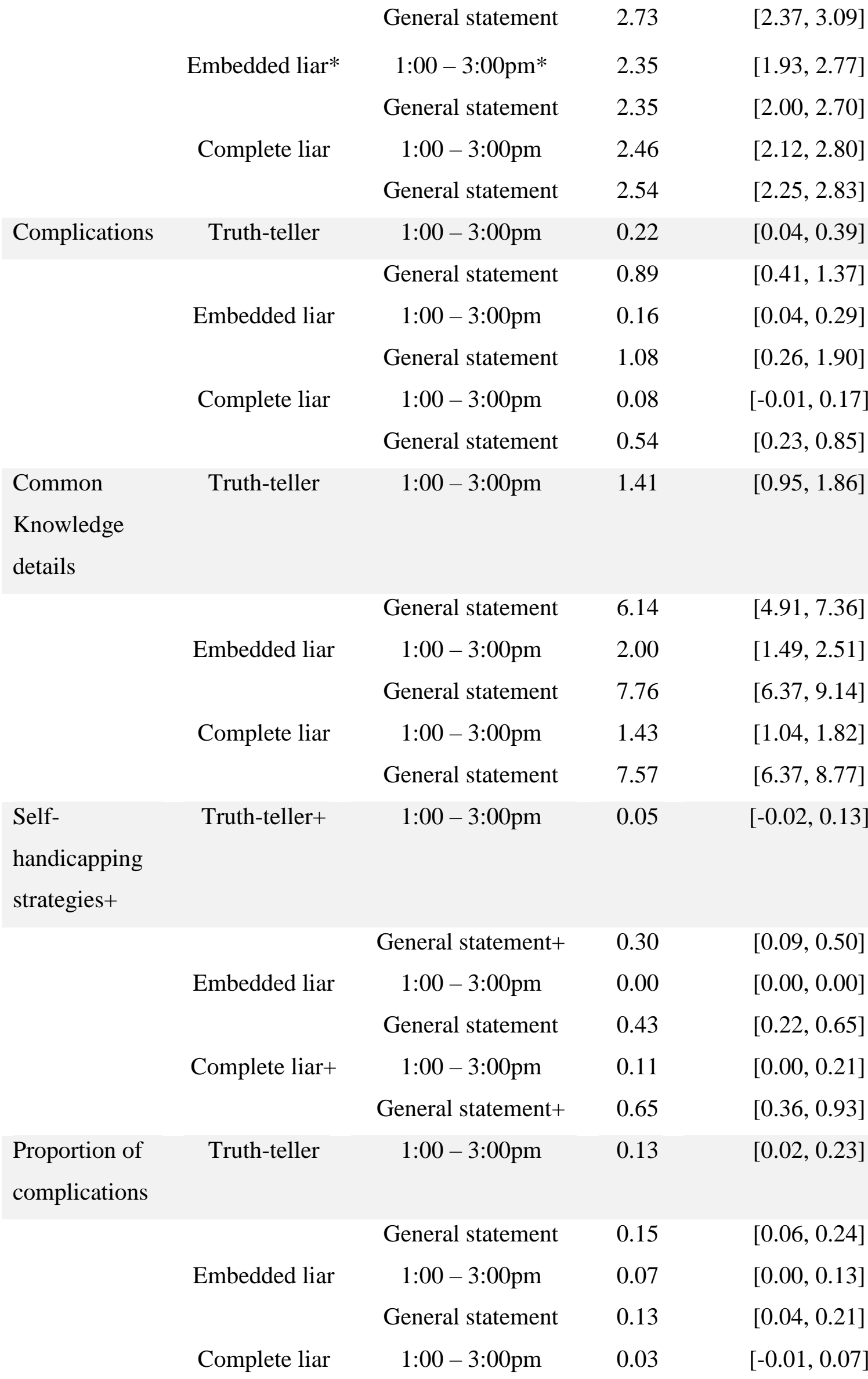




$$
\text { General statement } \quad 0.07 \quad[0.02,0.11]
$$

Note. The asterisks $(*)$ indicate the dependent variables with statistically significant differences for the 1:00 to 3:00pm critical component and where these differences occurred. The plus signs $(+)$ indicate the dependent variables with statistically significant differences for the general component and where these differences occurred.

We tested Hypothesis 4 by conducting a second series of univariate between-subjects ANOVAs to compare the 1:00pm to 3:00pm period between the Embedded lie and Complete lie conditions on the same secondary content cues. Only one significant difference emerged: Liars in the Complete lie condition provided some self-handicapping strategies $(M=.11, S D=$ .32) during the critical portion of the alibi, whereas those in the Embedded lie condition did not provide any, $F(1,72)=4.36, p=.040, \eta_{\mathrm{P}}^{2}=.057, B F_{10}=1.53$. We did not observe a significant effect for the remaining variables: The amount of verifiable details, $F(1,72)=1.26, p=.265$, $\eta_{\mathrm{P}}^{2}=.017, B F_{01}=2.42$; the number of complications, $F(1,72)=1.13, p=.292, \eta_{\mathrm{P}}^{2}=.015$, $B F_{01}=2.57$; the number of common knowledge details, $F(1,72)=3.22, p=.077, \eta_{\mathrm{P}}^{2}=.043$, $B F_{01}=1.05 ;$ the proportion of complications, $F(1,72)=0.77, p=.382, \eta_{\mathrm{P}}^{2}=.011, B F_{01}=2.98$; the quality of statements, $F(1,72)=.17, p=.685, \eta \mathrm{P}^{2}=.002, B F_{01}=3.88$. See Table 4.2 for the exact values. Thus, our analysis of the secondary content cues revealed no differences between lies embedded in truths and lies embedded in lies, with Bayes Factors demonstrating weak to substantial evidence in favour of the null hypothesis. The only exception was selfhandicapping strategies.

Exploratory testing. Having found that lies embedded in otherwise truthful statements did not differ from lies embedded in an otherwise deceptive statement, we were also interested to what extent these embedded lies could be distinguished from truths. We therefore conducted an exploratory analysis of the 1:00pm to 3:00pm period between the Embedded lie condition (lie embedded into a truthful statement) and the Truth-teller condition (truth also embedded into a truthful statement). The embedded truths $(M=21.22, S D=12.33)$ were significantly richer in detail than the embedded lies $(M=15.22, S D=9.65), F(1,72)=5.43, p=.023, \eta_{\mathrm{P}}^{2}=.070$, 
$B F_{10}=2.39$. Embedded truths $(M=2.95, S D=1.10)$ were also rated as having higher statement quality than embedded lies $(M=2.35, S D=1.25), F(1,72)=4.69, p=.034, \eta_{\mathrm{P}}^{2}=.061, B F_{10}=$ 1.76. We did not find significant differences for the remaining variables: The amount of verifiable details, $F(1,72)=3.74, p=.057, \eta_{\mathrm{P}}^{2}=.049, B F_{01}=0.85$; the number of complications, $F(1,72)=0.25, p=.616, \eta_{\mathrm{P}}^{2}=.004, B F_{01}=3.73$; the number of common knowledge details, $F(1,72)=3.12, p=.082, \eta_{\mathrm{P}}^{2}=.042, B F_{01}=1.10$; the number of selfhandicapping strategies, $F(1,72)=2.06, p=.156, \eta_{\mathrm{P}}^{2}=.028, B F_{01}=1.72$; the total proportion of complications, $F(1,72)=1.00, p=.321, \eta \mathrm{P}^{2}=.014, B F_{01}=2.71$. Exact values can be found in Table 4.2. These results indicate that lies embedded into otherwise truthful accounts can be differentiated from truths based on detail richness and statement quality, although the Bayes factors indicate the evidence for this is weak at best.

We preregistered our hypothesis that lies embedded in truths would be richer in detail than lies incorporated into fully fabricated accounts. It is, however, also possible that embedded lies affected the flanking truthful component. To investigate this, we conducted a series of independent samples $t$-tests on the dependent measures between the truthful portions flanking the embedded lies, and the same components flanking the truths. Embedded liars $(M=36.46$, $S D=17.91)$ and truth-tellers $(M=35.24, S D=20.74)$ provided similar richness of detail in the truthful components of their statements, $t(72)=-.27, p=.788, d=-.06, B F_{01}=4.03$. Similarly, we did not find significant differences for the remaining variables: The amount of verifiable details, $t(72)=.34, p=.735, d=.08, B F_{01}=3.96$; the number of complications, $t(72)=-.47, p$ $=.639, d=-.11, B F_{01}=3.78$; the number of self-handicapping strategies, $t(72)=-1.15, p=.253$, $d=-.27, B F_{01}=2.35$; the number of common knowledge details, $t(72)=-1.40, p=.165, d=-$ $.33, B F_{01}=1.79$. These results indicate that the truthful components of statements were consistently rich in detail, and included similar quality of detail, regardless of whether the statement was entirely truthful or contained an embedded lie. 


\section{Discussion}

In line with previous research (e.g., Amado et al., 2016; Luke, 2019), we found that entirely truthful statements were richer in detail compared to entirely fabricated statements (Hypothesis 1). In contrast to previous findings (Chapter 3 of this thesis), we did not find evidence that lies embedded into truthful stories were more richly detailed than lies embedded into completely fabricated stories (Hypothesis 2). Regarding our secondary dependent measures, self-handicapping strategies emerged as the only (weakly) diagnostic cue to differentiate entirely truthful statements from entirely fabricated statements (Hypothesis 3), and embedded lies from complete lies (Hypothesis 4).

This experiment investigated how the verbal content of lies was affected by embedding them into otherwise truthful statements. Our hypotheses, that lies embedded in truthful information would be richer in details (Hypothesis 2) and other high quality details (Hypothesis 4) than lies that are part of completely fabricated statements, were not supported. Consequently, lies embedded in otherwise truthful statements and lies embedded in deceptive statements could be distinguished from truths equally well. Our finding that lies embedded in truthful statements can be differentiated from truths that are part of fully truthful accounts is comparable to that of Gnisi and colleagues (2010). It is encouraging that in both studies, even when liars incorporate truthful, previously experienced information into their fabrications, differences still exist between these deceptive and truthful elements. This has important implications for practice, as it means that tools used in in the field such as Criteria-Based Content Analysis (CBCA; Steller \& Köhnken, 1989) may be robust to the influence of embedded lies.

Compared to being entirely truthful or entirely deceptive, telling a mixture of truths and lies could have resulted in reporting more details about the deceptive parts of their statement, fewer details about the truthful parts of their statement, or a combination of both. The lack of difference between the two types of lies suggests that embedded liars did not maintain 
consistency between the truthful and fabricated components of their statements. A potential explanation could be that, without specific knowledge of the criteria indicative of truthfulness, it would be difficult for liars to produce a fabricated element that is comparable in detail and quality to the truthful component. The only observable difference between the two types of lies was with regard to the presence of self-handicapping strategies; however, the observed significance can be explained by a floor effect meaning this result should be interpreted with caution. Additionally, we found that interviewees provided similar richness and quality of detail in the truthful components of their accounts, regardless of whether this component was flanked by truthful or deceptive information. This provides interesting insight into the high quality of statements that could potentially be provided by embedded liars.

When comparing the entire statement, completely truthful accounts differed from fully fabricated accounts, though only with regard to our primary cue richness of detail (Hypothesis 1) and self-handicapping strategies (Hypothesis 3). Importantly, richness of detail is the most empirically supported cue from the literature and therefore contributes strong insights to our pattern of results. Regarding our secondary cues, we failed to replicate previous findings that truthful accounts contain more verifiable details than deceptive ones. A potential explanation for the discrepancy between our findings and the general verifiability literature is that we did not employ the entire VA procedure (see also Bogaard, Meijer, \& Van der Plas, 2019; Nahari et al., 2014b). Research suggests that the VA approach is most effective when, prior to their interview, interviewees are requested to include details that the investigator can check - what is known as the information protocol (Harvey, Vrij, Nahari, \& Ludwig, 2016; Nahari et al., 2014b). We opted not to use this protocol because this instruction may have affected liars' and truth-tellers' responses and influenced the other dependent measures of our experiment.

We also did not find the proportion of complications to be a diagnostic cue to veracity in any of the three comparisons. This may have been due to floor effects in our sample (truthful 
interviewees in our study reported on average, less than one complication, relative to other studies in which truthful reports typically produce an average of ten or more complications; e.g., Vrij, Leal, Jupe, et al., 2018; Vrij, Leal, Mann, et al., 2017). Regarding common knowledge details, it has been suggested that truthful interviewees sound scripted in their reports if they underestimate the amount and type of detailed information they are required to report (Vrij, 2018). A potential reason for not observing significant differences with regard to the proportion of complications was due to the reduced time period, as well as the events, that participants were reporting in their alibi statement. If participants had reported longer, more dynamic statements, perhaps after exposure to a model statement (e.g., Leal et al., 2015), then the proportion of complications may have been a more effective cue.

We also did not observe differences regarding the statement quality between completely truthful statements and completely fabricated statements when comparing the entire accounts, yet we did replicate this effect when comparing only the embedded 1:00pm to 3:00pm component of the alibis. Reporting truthfully involves retrieving and reconstructing one's memory, whereas constructing a lie involves fabricating a story based on scripted knowledge about comparable situations and events (Schank \& Abelson, 1977). Considering that liars in our study admitted having included some truthful information in their statements, it is possible that this allowed their overall statements to come across equally as clear and plausible as honest interviewees.

\section{Limitations and Future Research}

The goal of our study was to examine embedded lies and we did so by isolating a critical statement of interest while manipulating the veracity of the surrounding components. However, the period for which the liars came up with spanned two hours. In real life, liars may stay as close to the truth as possible, only fabricating or omitting a few key, incriminating details. Future research could extend our paradigm to accommodate for the dispersion of truths and lies 
throughout a statement and particularly how interviewees' verbal content may be inconsistent when they lie and tell the truth in a single account.

A second limitation is that the self-reported truthfulness ratings revealed that liars instructed to fabricate their entire account reported still including some truthful information, and the embedded liars reported that their general statement was mostly truthful, but still included some lies. This may be methodologically somewhat awkward but it does reflect what liars typically do: Providing statements that contain a mixture of truths and lies (Leins et al., 2013, Leins, Zimmerman, \& Polander, 2017). As such, the finding is high in ecological validity. We did, however, check by self-report that liars did not engage in the assigned activity on the day in question nor on any adjacent days. This does not, of course, exclude the possibility that they engaged in the activity on an earlier occasion, meaning they could still have drawn from this truthful experience, simply displacing it in time. Future research that manipulates the type of lie that interviewees provide, such that it cannot be readily drawn from a potential previous experience, may produce a different pattern of results. Another methodological adjustment that may yield different findings would be allowing participants to choose the topic of their report, rather than constraining their reports to an activity scripted by the experimenter. This would more appropriately reflect the circumstances of real world liars, who are typically not forced to report any particular event (e.g., Leins et al., 2013).

Another important consideration relates to ground truth. Our study involved interviewees reporting self-generated stories within a naturalistic alibi scenario. We established partial ground truth via our truthfulness measures, which indicated that participants largely conformed to the experimental instructions. We were unable to further corroborate participants' accounts, however. Although the current experiment ensured that participants were emotionally engaged with the experimental process and similar paradigms have been used extensively by deception researchers (e.g., Elntib, Wagstaff, \& Wheatcroft, 2015; Masip et al., 2005; Sporer 
\& Sharman, 2006), future research would benefit from attempting to establish ground truth. A possible way to do so without having to resort to artificial mock crime procedures would be to require participants to wear a video-recording device for a certain duration of hours over a period of several days. Then, the researcher could verify the veracity of the interviewees' reports in the subsequent interview (e.g., Meixner \& Rosenfeld, 2014).

\section{Conclusion}

In sum, we showed that truthful statements could be distinguished from fabricated ones, and that lies embedded in otherwise truthful statements did not differ from lies embedded in deceptive statements. We also showed that lies embedded in otherwise truthful statements could be distinguished from truths embedded in truthful statements. Accordingly, verbal credibility assessment tools based on verbal content measured in this study may be robust against the embedding of lies. 


\section{Chapter 5: A Within-Statement Baseline}

\section{Comparison for Detecting Lies}

This chapter was published as:

Verigin, B. L., Meijer, E. H., \& Vrij, A. (2020). A within-statement baseline comparison for detecting lies. Psychiatry, Psychology and Law. doi: 10.1080/13218719.2020.1767712 


\begin{abstract}
To make veracity judgements in individual cases, practitioners may rely on baselining. That is, they may evaluate a statement relative to a baseline statement that is known to be truthful. We investigated whether a within-statement verbal baseline comparison could enhance discriminatory accuracy. Participants $(n=148)$ read an alibi statement of a mock suspect and provided a veracity judgement regarding a critical two-hour period within the alibi statement. This critical element was either deceptive or truthful and was embedded into an otherwise truthful story. Half of the participants received additional instructions to use the surrounding truthful elements of the statement as a baseline. Instructing participants to make a withinstatement baseline comparison did not improve the accuracy of credibility assessments.
\end{abstract}




\section{Introduction}

Deception researchers typically report their results as average scores from groups of participants. This research shows that, on average, liars' statements are less richly detailed than those of their truth-telling counterparts (e.g., Amado, Arce, Fariña, \& Vilarino, 2016; DePaulo et al., 2003; Luke, 2019). Legal practitioners, in contrast, are rarely interested in such group averages. They need to know whether an interviewee in the case at hand is being deceptive or honest. However, group-derived estimates do not always reliably generalize to individual cases (Fisher, Medaglia, \& Jeronimus, 2018; Faigman, Monahan, \& Slobogin, 2014).

One option that facilitates decisions at the individual level is to include a withinindividual comparison (see Vrij, 2016 for a discussion). One such method, reportedly used in practice by some police (Ewens, Vrij, Jang, \& Jo, 2014; Frank, Yarbrough, \& Ekman, 2006; Inbau, Reid, Buckley, \& Jayne, 2013; U.S. Department of the Army, 2006) is the baseline technique. With this technique, interviewers evaluate an interviewee's 'statement of interest' (i.e., part of the statement for which veracity is being assessed) relative to a baseline statement (i.e., part of the interview that is known to be truthful). Deception is then determined by looking for deviations from this established baseline. Moreover, $71 \%$ of experienced human intelligence interviewers reported to rely on deviations from baseline to detect deception (Russano, Narchet, Kleinman, \& Meissner, 2014).

A reason to believe in the efficacy of baselining as a lie detection technique derives from early research on the relationship between familiarity and deception. A handful of studies from the late $20^{\text {th }}$ century examined how the level of familiarity between a liar and an observer affects lie detection outcomes (e.g., Brandt, Miller, \& Hocking, 1980a, 1980b, 1982; Comadena, 1982; Ekman \& Friesen, 1974; Feeley, deTurck, \& Young, 1995; Hayano, 1980; McCornack \& Parks, 1986). Collectively, this research showed that veracity judgements were most accurate when observers had the opportunity to become familiar with the respondents' 
truthful communication style. For example, Feeley et al. (1995) had participants judge the veracity of truthful and deceptive communicators after viewing between zero and four exposures of the sender. They found a positive linear relationship between the amount of familiarity with the sender and judges' accuracy. In their meta-analysis, Bond and DePaulo (2006) compared deception detection accuracy between judges who had, versus those who had not, been previously exposed to the individual they were evaluating. Their results showed that when judges had been previously exposed to a target, this baseline exposure or baseline familiarity resulted in a small yet significant increase in detection accuracy from $52 \%$ to $56 \%$. These results support the role of baseline familiarity - that is, when the sender is familiar to the receiver, such as in personal relationships.

In police interviews, interviewer and target are more likely to be strangers, and another option is to use part of the same interview as the baseline statement. Ewens and colleagues (2014) examined the behavioural patterns of interviewees in response to an initial nonthreatening 'small-talk' baseline question ('You just read and signed an informed consent form, could you please tell me what you remember about it and what it said') compared to their behaviour in response to investigative questions, for which they knew their veracity would be assessed. The results indicated no effect of this baseline: Both truthful and deceptive interviewees behaved equally different between the small-talk baseline and investigative part of the interview. Palena, Vrij, Caso, and Orthey (2018) examined two types of baseline: An initial small-talk baseline and a comparable truth baseline (i.e., a set of questions designed to be comparable with investigative questions in terms of, for example, content, stakes and timeframe; Ewens et al., 2014; Vrij, 2008). They compared similarities in participants' nonverbal and verbal behaviours when responding to baseline and investigative questions, using the two types of baseline. They found that liars and truth-tellers in the small-talk baseline condition did not differ in their level of similarity between the baseline and investigative questions, adding 
further evidence to the ineffectiveness of this approach. Their results did, however, reveal that truth-tellers showed significantly more similarity than liars in the comparable baseline condition, though only in terms of spatial details.

Two previous studies examined the effect of verbal baselining on observers' accuracy rates. Caso, Palena, Carlessi, and Vrij (2019) tested police officers' ability to assess credibility when provided with a comparable truth baseline compared to when no baseline was provided. No differences were found for total and truth accuracy between conditions, although observers who made a baseline comparison did obtain higher lie detection accuracy rates. Caso, Palena, Vrij, and Gnisci (2019) found more promising results. These authors looked at the effects of small-talk and a comparable truth baseline on laypeople's deception detection accuracy. This study revealed that (i) participants in the comparable truth condition outperformed those in the small-talk condition in terms of total accuracy rates, and (ii) only observers who used a comparable truth baseline performed significantly better than chance levels in their total accuracy for distinguishing truth-tellers from liars.

Taken together, previous research on baselining shows that to enhance diagnostic accuracy, a comparable truth baseline should be used. That is, the baseline statement must be equivalent to the statement of interest in terms of content, time-frame, stakes, cognitive and emotional involvement, and questioning context (Caso, Palena, Vrij, \& Gnisci, 2019; Ewens et al., 2014; Palena et al., 2018; Vrij, 2008). Despite the importance of the baseline statement being equivalent to the target portion of the statement, each of the previous studies compared the effect of an initial, separate baseline statement to a target portion of an investigative interview. Investigating whether a baseline statement could be derived from parts of the interviewee's statement could have important implications for practitioners who may be inclined to draw such comparisons between corroborated and uncorroborated portions of an interviewee's account. 
The objective of the present study was to investigate whether introducing a withinstatement baseline comparison could improve the accuracy of participants' veracity judgements. Participants read the alibi statement of a mock suspect and provided a veracity judgement regarding a critical two-hour period within the alibi statement. This critical element was either deceptive or truthful and was embedded into an otherwise truthful story. We examined whether providing an instruction to utilize a comparable baseline (i.e., informing participants that all information, with the exception of the critical element, has been confirmed to be truthful) could enhance participants' detection accuracy. We hypothesized that participants who received the baseline instruction would have more accurate veracity judgements than participants who did not receive the baseline instruction.

\section{Method}

\section{Participants}

The sample consisted of 148 adult participants (120 females; 28 males) between the ages of 17 and 45 years $\left(M_{\text {age }}=20.53\right.$ years, $\left.S D=3.17\right)$. Our sample size was calculated prior to data collection by multiplying the total number of statements $(n=74)$ by two, ensuring that each statement was evaluated twice. Given this sample size, and an $\alpha$ of .05 , we had an $85.6 \%$ chance of rejecting the null hypothesis if there was a medium effect size $(f=.25$; Cohen, 1988). Only participants who were proficient in reading and writing English were eligible for the study. They were compensated with either course credit or a $€ 5$ voucher. The study was approved by the standing ethical committee. The study was pre-registered and approved via the Open Science Framework (http://j.mp/2IjvL51).

\section{Statements}

The statements that participants evaluated were previously collected by the Principal Investigator (see Chapter 4). These statements represent accounts provided by student research participants who were instructed to provide oral alibi statements to convince an interviewer that 
they were innocent of a hypothetical crime. For the present study, we incorporated the statements that were entirely truthful recollections of an interviewee's events on a particular day $(n=37)$, and the statements that were truthful accounts containing a lie from 1:00pm to 3:00pm $(n=37)^{7}$. For the latter group, interviewees truthfully reported their events on the day in question, before 1:00pm and after 3:00pm; however, during the critical element (i.e., between 1:00pm and 3:00pm) they were instructed to fabricate a particular activity. Thus, participants in the current study assessed one transcript that contained a critical element that was either deceptive (i.e., embedded into an otherwise truthful account) or truthful (i.e., part of an entirely truthful account). Each of the 74 statements was evaluated twice by two independent participants.

Ground truth. We attempted to establish partial ground truth of the statements by asking participants to self-report the truthfulness of both elements of their statement (on a scale of 1 to 10,1 being not at all truthful and 10 being completely truthful). Truth-tellers reported that both their general alibi $(M=9.32, S D=0.88)$ and the critical element $(M=9.59, S D=$ 0.90) were almost completely truthful. Those who provided the embedded lie reported that their general alibi was almost entirely truthful $(M=7.92, S D=2.45)$ whereas the critical element was mostly deceptive $(M=2.62, S D=2.48)$. The complete statistical analyses are reported in Chapter 4 (pp. 72-73). Overall, interviewees appeared to have largely conformed to the instructions they received across conditions.

\section{Design}

The experiment followed a between-subjects factorial design: 2 (Baseline instruction: Present vs. Absent) x 2 (Veracity of the critical element: Truth vs. Lie). Participants were randomly assigned to one of the four conditions. To mimic real-life investigations in which investigators typically only have one statement to assess, each participant judged only one

\footnotetext{
${ }^{7}$ The interviews included in the present study were a mean length of 4 minutes and 28 seconds $(\mathrm{SD}=2.50$; range: 1 minute and 50 seconds to 15 minutes and 22 seconds).
} 
statement. The dependent measure was the accuracy of participants' veracity judgements. Two accuracy scores were created by recoding participants' binary and Likert scale truth-lie judgements with the ground truth of the veracity of the critical element.

\section{Procedure}

Participants arrived at the lab and provided informed consent. Afterwards, they received a detailed instruction letter (see Appendix 4) explaining that their task was to imagine themselves in the role of a police detective who was investigating a violent burglary that occurred recently. Participants were told that the suspect was interviewed by police and had provided an alibi statement for the entire day in question, from morning to evening. They were informed that the critical element of the alibi was from $1: 00 \mathrm{pm}$ to $3: 00 \mathrm{pm}$ on this day. The critical element within each transcript was highlighted yellow to ensure this was clearly understood. Participants were instructed to read the entire statement carefully, but to make an assessment regarding the veracity of only the highlighted critical element. All participants were told that it was important to make the correct decision because it would earn them a chance to win $€ 50$ from a raffle draw.

Participants who were assigned to the Baseline-present condition received additional instructions prior to reading the transcript. They were informed that as the lead investigator, they had access to other sources of information for the case and this collateral evidence confirmed that the 'general' alibi statement, before 1:00pm and after 3:00pm was truthful (participants were not actually provided this collateral evidence). Participants were instructed to use this knowledge to compare the 'general' portion of the interviewees' alibi to the 'critical element from 1:00pm to $3: 00 \mathrm{pm}$ '. They were asked to try to identify any patterns or changes in the verbal content between the general alibi and the critical element that may indicate how credible the suspect's account was during the highlighted critical element. 
After reading the instructions, all participants received one written transcript of a suspect's alibi statement and they were given up to 10 minutes to read it. We used written transcripts to allow for highlighting the critical element in yellow. After reading the transcript, participants were prompted to first provide a binary veracity judgement (lie or truth) regarding the highlighted critical element. Then, they rated their veracity judgement on a 7-point Likert scale $(1$ = completely truthful to 7 = completely deceptive $)$. Subsequently, participants were prompted (i) to provide an open-ended description of the verbal cues they used to form their veracity judgement and (ii) to select veracity cues from a predetermined list. To preserve manuscript length, the coding and analyses of participants' veracity cues are reported in the Supplementary Materials. Once completed, participants responded to a short questionnaire that included a motivation check, general study experience questions and demographics information (i.e., age, sex, race, native language and education). Upon finishing, participants were debriefed and the study was concluded. All participants were entered into the $€ 50$ raffle, regardless of the accuracy of their veracity judgements. Participation in the study took approximately thirty minutes.

To evaluate the accuracy of participants' veracity judgements, the binary truth-lie judgements were transformed into incorrect and correct veracity decisions, according to the ground truth.

\section{Results}

\section{Motivation, Experimental Realism, and Self-Perceived Lie Detection Ability}

Several 2 (Baseline instruction: [present, absent]) X 2 (Veracity of the critical element: [truth, lie]) ANOVAs were conducted on participants' responses to a series of 7-point Likert scales $(1=$ not at all, $7=$ very $)$. These analyses revealed that participants, on average, were highly motivated $(M=6.29, S D=0.87)$, with no significant differences between baseline and veracity conditions, $F(3,144)=1.07, p=.366, \eta_{\mathrm{P}}^{2}=.022$. On average, participants reported to 
answer questions honestly $(M=6.82, S D=0.45)$, with no significant differences between baseline and veracity conditions, $F(3,144)=0.29, p=.830, \eta_{\mathrm{P}}^{2}=.006$. On average, participants found the instructions very clear $(M=6.49, S D=0.80)$, with no significant differences between baseline and veracity conditions, $F(3,144)=1.26, p=.290, \eta_{\mathrm{P}}^{2}=.026$. We also observed that participants, on average, found the statements realistic $(M=5.39, S D=1.24)$, with no significant differences between baseline and veracity conditions, $F(3,144)=0.35, p=.789, \eta \mathrm{P}^{2}$ $=.007$. Additionally, participants self-reported to be average lie detectors $(M=4.10, S D=1.14)$, with no significant differences between baseline and veracity conditions, $F(3,144)=1.11, p=$ $.345, \eta_{\mathrm{P}}^{2}=.023$.

\section{Veracity Judgements}

Participants' veracity judgements were analysed using a two-way between-subjects ANOVA on the Likert scale judgements, and a logistic regression on the binary data. In addition, we also subjected the binary judgements to a two-way between-subjects ANOVA. Although duplicate, we report this analysis to allow for a comparison with previous research and because it allows for the calculation of Bayes Factors, which helps with the interpretation of the results. The Bayesian factors (BF; for interpretation, see Jarosz \& Wiley, 2014; Lee \& Wagenmakers, 2013) are reported in line with the cut-off points outlined by Jeffreys (1961). The approximate evidence categories are as follows: Values between 1 and 3 indicate weak evidence for the alternate or null hypothesis, between 3 and 10 indicate strong evidence, and above 10 are considered very strong evidence. The interaction model within JASP combines both main effects and the interaction effect; therefore, evidence for the interaction term individually was calculated by dividing the interaction model by the main factors (e.g., Wagenmakers et al., 2016). For ease of interpretation, $B F_{10}$ is used to indicate the Bayes factor as evidence in favor of the alternative hypothesis, whereas $B F_{01}$ is used to indicate the Bayes factor as evidence in favor of the null hypothesis. 
Table 5.1 displays the means and standard deviations of both the binary and Likert judgements. Overall, the accuracy of participants' binary judgements, where 0 represents incorrect and 1 represents correct judgements, did not differ significantly from chance level ( $M$ $=0.53, S D=0.50, t(147)=0.82, p=.413, d=.06$. To examine whether the baseline instruction increased participants' ability to accurately discriminate between lies and truths, we conducted a 2 (Baseline instruction: [present, absent]) X 2 (Veracity of the critical element: [truth, lie]) between-subjects ANOVA on the accuracy of participants' binary veracity judgements. Contrary to our hypothesis, the main effect of the Baseline instruction was not significant, $F(1$, 144) $=0.26, p=.613, \eta_{\mathrm{P}}^{2}=.002 ; B F_{01}=5.06$, meaning that participants who received the Baseline-present instructions $(M=0.55, S D=0.50,95 \% \mathrm{CI}[0.44,0.67])$ were not significantly more accurate in their veracity judgements than participants who received the Baseline-absent instructions $(M=0.51, S D=0.50,95 \% \mathrm{CI}[0.40,0.63])$. This analysis revealed a main effect of the Veracity of the critical element $\left(F(1,144)=10.33, p=.002, \eta_{\mathrm{P}}^{2}=.067 ; B F_{10}=18.49\right)$, with lies $(M=0.66, S D=0.48,95 \%$ CI $[0.55,0.77])$ being judged more accurately than truths $(M=0.41, S D=0.49,95 \%$ CI $[0.29,0.52])$. Finally, the Veracity of the critical element by Baseline instruction interaction effect was also not significant, $F(1,144)=1.40, p=.238, \eta_{\mathrm{P}}^{2}$ $=.010 ; B F_{01}=2.32$, indicating that the baseline instruction had no differential effect on the accuracy of participants' veracity judgements for lies and truths.

The logistic regression on the binary judgement with predictor variables (Baseline instruction: [present, absent] and Veracity of the critical element: [truth, lie]) and the accuracy of participants' binary veracity judgements as the dependent measure, revealed a significant overall model, $\chi^{2}(2)=10.18, p=.006$, Nagelkerke $R^{2}=.09$. Contrary to our hypothesis, the Baseline instruction was not a significant predictor of classification accuracy $(p=.609,95 \% \mathrm{CI}$ $[.43,1.64])$. The Veracity of the critical element, however, was a statistically significant predictor $(p=.002)$ : Participants who evaluated a statement containing a deceptive critical 
element had 2.88 times higher odds (95\% CI [1.47, 5.63]) of making a correct veracity judgement than those who evaluated a statement containing a truthful critical element.

The 2 (Baseline instruction: [present, absent]) X 2 (Veracity of the critical element: [truth, lie]) between-subjects ANOVA on participants' Likert scale veracity judgements revealed no significant differences. We did not observe a significant main effect of the Baseline instruction, $F(1,144)=.95, p=.332, \eta^{2}=.007 ; B F_{01}=3.69\left(M_{\text {Baseline-present }}=4.27, S D=1.54\right.$, 95\% CI $[3.92,4.62]$ versus $M_{\text {Baseline-absent }}=4.03, S D=1.53,95 \%$ CI $\left.[3.68,4.38]\right)$. Nor did we find a significant main effect of the Veracity of the critical element, $F(1,144)=.75, p=.388$, $\eta_{\mathrm{P}}^{2}=.005 ; B F_{01}=4.04\left(M_{\text {Lies }}=4.26, S D=1.50,95 \% \mathrm{CI}[3.91,4.61]\right.$ versus $M_{\text {Truths }}=4.04, S D$ $=1.57,95 \%$ CI $[3.69,4.39])$. Finally, the Veracity X Baseline interaction effect was not significant, $F(1,144)=3.80, p=.053, \eta_{\mathrm{P}}^{2}=.026 ; B F_{01}=0.84$.

Table 5.1

Descriptive statistics of binary and Likert veracity judgements across conditions

\begin{tabular}{lcccc}
\hline & \multicolumn{2}{c}{ Baseline instruction } & & \multicolumn{2}{c}{ No baseline instruction } \\
& $\begin{array}{c}\text { Deceptive } \\
\text { element }\end{array}$ & $\begin{array}{c}\text { Truthful } \\
\text { element }\end{array}$ & $\begin{array}{c}\text { Deceptive } \\
\text { element }\end{array}$ & $\begin{array}{c}\text { Truthful } \\
\text { element }\end{array}$ \\
\hline Binary Judgement & $.73(.45)$ & $.38(.49)$ & $.59(.50)$ & $.43(.50)$ \\
Likert Judgement & $4.62(1.44)$ & $3.92(1.57)$ & $3.89(1.49)$ & $4.16(1.57)$
\end{tabular}

Note. Means; Standard deviations in parentheses. The binary judgements have been recoded for accuracy, whereas the Likert scores are in their original form.

\section{Discussion}

It is well documented that observed lie detection rates hover around 50\% (e.g., Bond \& DePaulo, 2006). We replicated this finding. We found that when making a binary judgement, participants were significantly better at detecting lies than at detecting truths. Contrary to our hypothesis, however, we found that participants who were instructed to utilize a within- 
statement comparable baseline did not outperform the control group in terms of overall lietruth discrimination accuracy.

Previous research revealed that the comparable truth baseline technique can enhance observers' judgement accuracy (Caso, Palena, Vrij, \& Gnisci, 2019). A possible explanation for the divergence in findings between this study and our own stems from how the statements were generated. In the experiment by Caso, Palena, Vrij, and Gnisci (2019), interviewees reported about experimental tasks they had completed. Our study, in contrast, used statements about participants' experienced activities on a particular day. Consequently, our participants were mostly unconstrained in their reports. These paradigms differ systematically in the source of deception, which was either scripted by the researcher (Caso, Palena, Vrij, \& Gnisci, 2019, i.e., a scripted task; Vrij, 2008) or drawn freely from the participant's own experience (current study, i.e., an autobiographical task; Sporer \& Sharman, 2006). When lies are self-generated, the deceiver can elaborate with personal experience, whereas lies designed by the researcher cannot be so easily embellished. As a consequence, it is possible that the statements resulting from the different sources of deception were perceived differently by the lie-detectors. Scripted tasks, relative to autobiographical tasks, may have been more straightforward to evaluate, which perhaps contributed to the incongruent findings between the present study and that of Caso, Palena, Vrij, and Gnisci (2019).

A second explanation for these results could be derived from our ground truth manipulation check. Compared to interviewees who reported entirely truthful recollections of their day, interviewees who embedded a lie reported lower ratings of truthfulness for the general, truthful portion of their statement. Thus, the truthful baseline of liars' statements may have been more comparable with their lies, which could have weakened lie-detector's ability to make accurate decisions. Although this may accurately reflect real-world conditions in which interviewees interweave truths and lies (e.g., Vrij, 2008), for the purposes for testing the 
efficacy of within-statement baselining future research should utilize a more controlled method for establishing baseline truthfulness.

The analysis of variance of the binary veracity judgements allows for a comparison to the results of previous work (Caso, Palena, Vrij, \& Gnisci, 2019). These authors found that using a comparable truth baseline led to significantly above-chance levels in total accuracy for distinguishing truth-tellers from liars $(d=.34)$. We found no effect of the baseline instruction with a Bayes Factor indicating substantial evidence for the absence of an effect. To directly compare to previous research, we also calculated a Cohen's d effect size, which reaffirmed a very small effect $(d=0.08)$ of the baseline instruction on participants' binary judgements. The finding from the Likert judgements are, however, more ambiguous. This measure also indicated no effect of the baseline instruction, but the veracity by baseline interaction revealed a $p$ value of .053 and was accompanied by an inconclusive Bayes Factor of 0.84 in favor of the null hypothesis. This pattern suggests that although we did not find evidence that the withinstatement baseline comparison was an effective lie detection tool, we also cannot rule out that it may have an effect that was too small for our study to pick up. Future research should replicate our study with an increased sample size. Although our power analysis indicated that we had sufficient power to detect an effect size of similar nature of Caso, Palena, Vrij and Gnisci (2019) had it been present, we may have been underpowered to detect a smaller effect size.

Current methods of verbal baselining may require further refinement before they can be reliably used to detect deception. In two studies to date (the current study and Caso, Palena, Carlessi, \& Vrij, 2019), instructing observers to make comparisons of the verbal content between known-truthful and target portions of a statement did not improve the accuracy of credibility assessments. In both experiments, participants in the baseline conditions were invited to search for deviations or patterns that might be indicative of deceit. Alternatively, explicitly training participants to look for empirically valid verbal deception cues, such as the 
richness of detail (e.g., Luke, 2019) or the verifiability of information (e.g., Nahari, Vrij, \& Fisher, 2014), may increase the effectiveness of baselining.

In sum, we did not find evidence that the within-statement baseline technique can enhance deception detection accuracy. Additional work is required to refine the technique and determine its true efficacy across different contexts. 


\section{Chapter 6: \\ General Discussion}




\section{General Discussion}

To date, most deception research treats veracity as a dichotomous factor. This is despite the growing body of evidence that truthful and deceptive information often exist within the same statement (e.g., Leins et al., 2013, 2017; Nahari, 2018a; Nahari \& Vrij, 2015). Accordingly, the overarching aim of the current thesis was to provide an empirical examination of lies that are embedded into truthful statements. In particular, the studies conducted within this thesis explored three factors: (i) how individuals who describe themselves to be good liars use strategies to successfully evade detection, (ii) to what extent embedded lies affect the verbal quality of statements, and (iii) if embedded lies can be exploited to facilitate lie detection within statements. This discussion section provides an overview of the key findings and a consideration of the theoretical and practical implications. Methodological considerations of the current research are presented as well as avenues for future research.

First, we explored how self-reported good liars use strategies to successfully evade detection. Meta-analytic findings indicate that the success of uncovering a deceptive interaction depends more on the liar than on the lie detector (e.g., Bond \& DePaulo, 2008). Yet, little is known about what enables good liars to evade detection. Thus, the primary aim of the research presented in Chapter 2 was to explore the lie prevalence and deception strategies of self-reported skilled liars. Our results replicated earlier research that a minority of individuals account for the majority of lies told in daily life, and we found evidence that these prolific liars also consider themselves good liars. Importantly, we also observed that self-reported good liars use verbal strategies of deception, with their most common strategy being to embed lies into truthful stories. This chapter highlighted the importance of developing strategy-based interventions that account for the strategic embedding of lies, particularly by skilled liars.

Second, we examined how embedded lies may affect the verbal quality of statements. Chapter 3 reported an experimental examination of how interviewees strategically regulate the 
information they provide when their accounts contain both truths and lies. We found that interviewees calibrate the richness of detail provided in the first element of their statement based on the veracity of the following element, such that elements followed by a lie were less detailed than elements followed by a truth. Moreover, we showed that lies become more detailed when they are flanked by truthful information compared to when they are flanked by other lies, meaning that interviewees also calibrate their lies according to both the preceding and the following element. The findings reported in this chapter suggest that when statements contain both truthful and deceptive components, liars are able to strategically calibrate the richness of detail.

Given our calibration evidence, the following study (Chapter 4 of this thesis) manipulated statements to contain lies embedded into truthful stories. We then investigated whether embedded lies differed qualitatively compared to full fabrications. This study revealed that lies embedded in otherwise truthful statements did not differ from lies embedded in deceptive statements in terms of, for example, the richness of detail and verifiability of information. Moreover, we showed that embedded lies could be distinguished from truthful statements based on detail richness and statement quality (i.e., clarity and plausibility). Based on these findings, verbal credibility assessment tools that rely on the verbal content measured in this study may be robust against the embedding of lies.

Third, we evaluated whether embedded lies could be exploited to facilitate lie detection within statements (Chapter 5). We expanded on earlier research on the baseline technique by exploring whether introducing a within-statement verbal baseline comparison could enhance discriminatory accuracy between lies and truths. Our results suggested that instructing participants to make a within-statement baseline comparison did not improve the accuracy of deception detection. This chapter thus informed our understanding of within-statement lie 
detection, with applied relevance given police officers' potential reliance on similar techniques during investigations.

\section{Main Findings of the Thesis}

Truths and lies within statements. The findings of the current research lend support to the small body of earlier work showing that, when given the opportunity, liars will incorporate as much truthful information as possible into their accounts (e.g., Leins et al., 2013, 2017; Nahari, 2018a; Nahari \& Vrij, 2015; Nahari et al., 2014b; Vrij, 2008; Vrij, Granhag, \& Porter, 2010). In theory, if liars draw on their memory traces of truthful previous experiences to report perceptual and contextual information, then their statements may resemble those of truth-telling interviewees. In turn, this could affect verbal credibility assessment tools that are derived by the assumption that lies have different content patterns than do truths (e.g., CBCA and RM). This thesis provided evidence that self-reported good liars report embedding their lies as a strategy for successfully deceiving (Chapter 2). We also showed that when lies are flanked by truthful information, they become more detailed (relative to when they are flanked by more deceptive information; Chapter 3). A promising finding - that speaks to the potential robustness of verbal veracity assessment tools such as CBCA and RM against embedded lies - was that lies embedded in truthful stories did not differ qualitatively from lies that were incorporated into fully fabricated statements on a number of cues (e.g., detail richness and verifiability of information; Chapter 4). This finding strengthens earlier work showing that verbal lie detection tools are still effective even when liars incorporate previous experiences (Gnisci et al., 2010; Valois et al., 2019), which has relevance for deception scholars and legal professionals alike.

Two of our experimental studies involved the production of lies. We instructed participants to fabricate their stories (Chapters 3 and 4), yet we found evidence that their lies were blended with truthful information. In Chapter 3, participants across veracity conditions reported to have strategically included truthful details that they had drawn from previous 
experiences and/or memory, meaning that we had deceptive statements that were likely a combination of truths and lies. Similarly, in Chapter 4, the self-reported truthfulness ratings revealed that liars instructed to fabricate their entire account reported still including some truthful information, and the embedded liars reported that their general statement was mostly truthful, but still included some lies. These findings, although somewhat difficult to disentangle in an experimental sense, contribute further evidence that liars provide statements that are a blend of truths and lies.

Liars' tendency to include truthful information in their deceptive accounts could be explained by the construction of lies. Although many theories of deception have presumed that lies and truths result from different production processes (i.e., the theoretical groundwork for CBCA and $\mathrm{RM}$ ), it is also possible that lies are typically built from variations of the truth or a related episodic memory of an event, either personally or vicariously experienced (Sporer, 2016; Walcyzk, Harris, Duck, \& Mulay, 2014). This paradigm would suggest that instead of liars carefully selecting units of information to include in their lies, lies might be constructed from the most readily accessible information within their working and/or long-term memory (McCornack, Morrison, Paik, Wisner, \& Zhu, 2014). Indeed, some have argued that truths and lies stem from the same speech production system (Mohamed et al., 2006; Walczyk et al., 2014). Thus, when we ask participants to lie in our experiments, they may simply draw on the first thing that comes to mind, which is likely to be a previous experience.

Given the tendency of liars to include truthful information within their deceptive reports, in combination with previous research findings (Leins et al., 2013) and those presented in this thesis, it is feasible that researchers have already been inadvertently examining embedded lies. That is, rather than comparing the extent to which truthful statements differ from deceptive statements, perhaps we have been investigating the differences between truthful accounts and partial lies that are under the guise of complete fabrications. This also offers a possible 
explanation as to why lies and truths are notoriously difficult to differentiate. If guilty suspects provide statements that are predominantly truthful, with only a few self-incriminating aspects modified, then it further complicates the credibility assessors' task (Gnisci et al., 2010; Vrij, 2008a). Even so, supposing that deception research has already been examining lies that are embedded with truthful information, it stands to reason that the findings from the general deception literature also pertain to embedded lies. For example, it was previously shown that CBCA and RM are relatively effective tools as, with appropriate training, evaluators' accuracy rates increase from chance level to between $65 \%$ and $80 \%$ (Vrij, 2008a). Indeed, this finding may be the product of studies that were distinguishing truthful statements from deceptive statements that contained truthful information. If that was the case, we can speculate that these tools may be informative whether the lie has been invented entirely or based upon a previous truthful experience.

In fact, research from this thesis demonstrated that embedded lies do not differ qualitatively from full fabrications, at least on a subset of criteria (Chapter 4). These lies embedded in otherwise truthful statements could be distinguished from truths embedded in truthful statements, which is comparable to the results of a previous study that examined how lies differ when they are invented or based on previous experience (Gnisci et al., 2010). In both studies (i.e., Chapter 4 of this thesis and Gnisci et al.'s 2010 experiment), even when liars incorporated truthful, previously experienced information into their fabrications, differences still existed between the deceptive and truthful elements. Similarly, in their unpublished work, Valois and colleagues (2019) examined whether CBCA and RM techniques could differentiate both fully and partially deceptive accounts from truthful accounts. They found that both tools were still effective even when interviewees incorporated truthful events into fabricated accounts.

An extension of the consistency framework. Much of the research presented in this thesis is grounded in the idea that liars are highly concerned with maintaining consistency within 
their statements (e.g., Deeb et al., 2017, 2018; Hartwig et al., 2010). Whilst the majority of previous research regarding consistency has referred to factual content, Deeb et al. (2017) provided empirical evidence that liars' attempts to maintain consistency generalise to other situations (i.e., including fewer repetitions in specific parts of their interview). Moreover, Leins et al. (2017) provided anecdotal evidence that liars' draw on previous experiences to report statements detailed in, for example, spatial information. The results of our first experiment (Chapter 3) contributed further evidence that liars' attempts to maintain consistency extend beyond the factual content and can influence the richness of detail provided. A notable finding was that liars calibrated the detail richness of their lies to be consistent with that of their truths.

However, when we extended our paradigm to include lies that were embedded into truthful information (Chapter 4), the pattern of consistency faded. That is, the lack of difference between the two types of lies suggested that embedded liars were not able to maintain consistency within the truthful and fabricated components of their statements, since the lies did not become more detailed to mirror the truths. Moreover, the truthful (non-target) portions of the statements were similarly rich in detail, regardless of whether the statement was entirely truthful or contained an embedded lie. It is possible that, if liars had specific knowledge of the criteria indicative of truthfulness, they may have been better able to produce a fabricated element that was comparable in detail and quality to the truthful component.

The research conducted in this thesis attempted to examine the verbal characteristics of embedded lies as a function of statement consistency. Taking our findings together, we found contradictory evidence regarding how liars manage the consistency of details between truths and lies within statements. A possible explanation as to why we observed the consistency effects in Chapter 3, but not in Chapter 4, relates to the cognitive load of liars. In the first experiment (Chapter 3), we examined the effect of two elements immediately following each other, whereas our next study (Chapter 4) examined a more true-to-life scenario in which a lie was embedded 
into an otherwise truthful alibi statement. It may have been easier for liars to calibrate the richness of details they provided when they could compartmentalise their reports about the two distinct and brief tasks (Chapter 3). In contrast, it may have been more cognitively demanding for liars to monitor the information they provided throughout a longer, more dynamic statement (Chapter 4). This additional cognitive load could have impaired liars' ability to strategically regulate the consistency of details between their truths and lies. Still, this pattern of results goes against common sense, as it would presumably be easier for liars to draw on their truthful memories when embedding a lie into their otherwise autobiographical memory versus when reporting a scripted task. Nonetheless, if the additional cognitive pressure of selectively drawing from memory traces does weaken liars' ability to maintain consistency within statements, it could mean that real world liars are unable to provide high quality, consistently detailed accounts when using embedded lies. This would contribute additional support to the reliability of verbal credibility assessment tools for distinguishing both fully and partially fabricated accounts. Researchers should continue exploring this extension of the consistency framework, given that both liars and legal professionals associate consistency with veracity (e.g., Deeb et al., 2017; Hartwig et al., 2010; Vredeveldt et al., 2014). Advancing our theoretical knowledge on consistency, or other potential verbal characteristics of embedded lies, is beneficial for designing strategy-based methods for exploiting these types of lies.

Exploiting embedded lies to facilitate deception detection. Research conducted in this thesis was, to our knowledge, the first to examine whether introducing a baseline comparison that was derived from multiple different parts of an interviewees' statement could facilitate the detection of lies (Chapter 5). Our paradigm provided an individual-level comparison and simultaneously allowed the opportunity to test whether embedded lies could be exploited to facilitate lie detection. Contrary to our hypothesis, we found that participants who were instructed to use a within-statement comparable baseline did not outperform the control group in terms of 
lie-truth discrimination accuracy. This contradicted the results of a previous study which suggested that the comparable truth baseline can enhance observers' ability to distinguish truthtellers from liars (Caso, Palena, Vrij, \& Gnisci, 2019).

An important limitation to consider is that the paradigm we tested, while controlling for variation at the individual level, did not control for varying events throughout the statement. A preferable method of verbal baselining is to generate baseline comparisons that control for both the individual and the situation (Vrij, 2016). Examples of this type of baselining could be to first have interviewees freely recall an event in chronological order and then to have them recall the same event in reverse order (Vrij, Leal, Mann, \& Fisher, 2012), or to obtain a free recall, administer a Model Statement, and then obtain a second statement of the same event (Leal et al., 2018). With these types of comparisons, investigators can look for deviations in the types of details (e.g., reminiscences, contradictions, complications or peripheral information) provided in the second statement, relative to the initial, baseline statement (Vrij, 2016; Vrij, Leal, \& Fisher, 2018). Additional within-subjects alternatives could be to evaluate the proportion of verifiable details (Nahari et al., 2014b; Vrij, 2016) or complications (Vrij, Leal, Jupe, \& Harvey, 2018) during an interviewees' statement regarding an event. Future research should continue exploring avenues of verbal baselining, improving our paradigm to control for both the individual and the situation.

The rationale for believing that baseline comparisons, that control for both the individual and situation, could help to exploit embedded lies relates to our consistency framework. It is possible to manipulate the cognitive load or cognitive flexibility of liars, for instance, by changing the report mode (e.g., Leins, Fisher, Vrij, Leal, \& Mann, 2011) or employing the reverse-order technique (e.g., Vrij et al., 2017). Such methods have shown to enhance deception detection based on statement consistency (Leins, Fisher, \& Vrij, 2012; Vrij, 2016). Specifically, introducing unanticipated changes in question format - such as asking suspects for an oral 
account and then for a sketch (Leins et al., 2012; Leins, Fisher, Vrij, Leal, \& Mann, 2011) or asking for a reverse order narration after a chronological report - reduces the levels of consistency in liars' statements more than in truth-tellers' statements. These techniques aid truthtellers in retrieving additional information about an event as they remember it from a different perspective, so called reminiscences (e.g., Leins et al., 2012). In contrast, realising that consistent accounts are more likely to be perceived as credible, liars are likely to repeat their first statement without adding any new information (e.g., Vrij, 2016). The complexity of reporting information in reverse order or via drawings may also generate more errors from liars in the form of contradictions between the two statements (Vrij et al., 2012). Thus, using within-subjects comparisons that exploit liars' attempts at maintaining consistency show promise as an avenue for deception detection, and it would be highly useful for future research to test these comparisons explicitly with embedded lies.

\section{Practical Implications}

The main practical implications from this thesis relate to the use of verbal credibility assessment tools and within-statement methods of deception detection. Based on the research conducted in this thesis, two recommendations can be offered to practitioners who conduct verbal credibility assessments.

CBCA is one of the most widely used credibility assessment techniques worldwide, and can be used as evidence in some courts in North America and in European countries such as the Netherlands, Germany and Sweden (Verschuere, Vrij, \& Granhag, 2015; Vrij, 2000). In the absence of evidence that embedded lies may undermine the efficacy of verbal veracity assessment techniques (e.g., Chapter 4 of this thesis; Gnsici et al., 2010; Valois et al., 2019), my tentative recommendation is for legal practitioners to continue using tools such as $\mathrm{CBCA}$ as investigative aids, when necessary. This recommendation comes with three caveats. First, practitioners should be particularly cautious making verbal credibility assessments during 
circumstances in which interviewees could easily draw upon truthful previous experiences. Although embedded lies may not render tools such as CBCA and RM entirely ineffective, these lies do have the potential to interfere with classificatory accuracy. Second, practitioners should prioritise richness of detail (i.e., the level of detail or amount of specific information included in a statement) as a cue to deception during statement analysis as this is one of the only robustly validated cues within the literature (e.g., Luke, 2019). Third, practitioners should, in general, be wary of their application of assessment outcomes from tools such as CBCA to legal decisions, given that these tools have a relatively high margin of error, even under favorable conditions (accuracy rates between $65 \%$ and $80 \%$; Vrij, 2008a). Verbal credibility assessment tools may be most advantageous during investigations or legal proceedings in which physical evidence is absent or inconclusive, whereas law enforcement resources may be more productively allocated to different avenues of investigation when available (e.g., evidence collection and corroboration).

Another practical implication relates to within-statement lie detection. Practitioners have repeatedly made valid requests for lie detection techniques to incorporate within-subjects comparisons; however, such comparisons are not very prevalent in the non-verbal and verbal lie detection fields (see Vrij, 2016 for a discussion). Evidence in this thesis (Chapter 5) showed that a within-statement verbal baseline comparison may not be an effective lie detection tool. This finding has applied relevance given police and intelligence officers' potential reliance on similar techniques during investigations (e.g., Ewens et al., 2014; Frank et al., 2006; Inbau et al., 2013; Russano et al., 2014). Based on these results, I would caution investigators from drawing comparisons between corroborated and uncorroborated portions of an interviewee's account in the same manner that we did. Instead, my recommendation is that practitioners utilise methods of baselining that control for both the individual and the situation.

\section{Methodological Considerations and Limitations}


A number of methodological considerations and limitations of the research conducted in this thesis warrant attention. Some have already been addressed throughout this chapter, and I will raise an additional five points. First, this research pertains to a specific type of embedded lie. To gain insight into liars' verbal behaviour and strategies when providing a mixture of truthful and deceptive information, we were particularly interested in examining deceptive events that were incorporated into truthful stories. This differs from other variations of embedded lies that may involve, for example, the omission of critical information without the offer of an alternative explanation, or embedded lies that contain only a small deceptive fragment (such as the date an activity occurred or a substitution of the perpetrator) whilst the remainder of the account remains truthful. In these latter variations of embedded lies, the interviewee does not provide a substantial lie and it thus becomes difficult to evaluate content-based cues within the truthful and deceptive portions of their statement. The extent to which these types of embedded lies may influence verbal lie detection tools thus remains unknown. We could speculate that these sorts of embedded lies are particularly problematic since the statements may be almost entirely truthful.

Similarly, mixed veracity statements are not solely provided by guilty individuals trying to evade detection. Innocent interviewees may also incorporate lies into their otherwise truthful stories for a variety of reasons, such as for impression management concerns (e.g., Colwell et al., 2018; Clemens \& Grolig, 2019). Lying by innocent suspects might lead to statement inconsistencies that are interpreted by investigators as cues to deception, thereby increasing their risk of being wrongfully assessed as guilty for a crime they did not commit. Future research should expand upon the research conducted in this thesis to account for various other types of embedded lies, including those from both the perspectives of guilty and innocent interviewees.

Second, two of our three experiments relied heavily on the verbal coding of statements. Although this is a favored methodology in verbal deception research, it is not without limitation. Guidelines exist for coding content-based cues, but there are a number of degrees of freedom 
afforded to researchers' development and execution of their own coding schemes. With this freedom comes the potential for a lack of consistency between different deception laboratories. In our studies, we reported moderate to high inter-rater reliability, suggesting that the consistency between raters was sound. However, there is still a possibility that different researchers may have drawn different conclusions from our same data set, had their coding criteria differed. This is a problem that relates to verbal lie detection research in general, and future work should develop more stringent and transparent guidelines for content-based coding that would help alleviate these issues. Researchers have proposed several ways to improve consistency in this field: (i) by requiring laboratories to specify their coding schemes and to make these, and other training, materials publically available; (ii) by organizing a cross-laboratory collaboration to examine the reliability and validity of various coding methodologies; and (iii) by searching for ways to combine automated scoring systems with human-driven, contextually-based methods (Nahari et al., 2019). Doing so would increase the reliability and generalisability of content-based deception research.

Third, a focus of the current thesis was on interviewees' strategies. We conducted extensive qualitative coding and analyses to open-ended responses from participants in Chapters 2 through 5. Our intention was to conduct a broad exploration of people's strategies in a variety of contexts, to inform our understanding of liars' metacognitions about deceiving. In theory, these insights could then be exploited in strategy-based lie detection techniques. However, this research method yielded less informative data than expected. Although we obtained a wide variety of responses at the individual level, our coding process required the reduction of responses into a practical number of categories that could be submitted to statistical procedures and that would produce meaningful results. This methodology led to the elimination of the more nuanced and informative units of data, and instead generated broader, less insightful categories of strategies. The data from our multiple-response questions regarding strategy use, however, 
were more informative and aligned to the behavioural data. Future research should aim to overcome this limitation of our work by prioritising directed questions regarding interviewees' strategies, and different qualitative coding methodologies may also be preferred.

Fourth, there are constraints to the generalisability of the experimental research conducted within this thesis (Chapters 3, 4, and 5). In particular, our experiments relied on samples of motivated university students. A drawback of this sample is that the findings may not be representative of the population in general, and therefore may not generalise beyond academic settings to legal contexts. Another common criticism of laboratory-based deception research is that researchers typically instruct people to lie, whereas in real life, people presumably elect whether to lie or tell the truth as well as the topics of those reports. Despite the artificiality of laboratory experiments, the primary advantage of this research methodology is the internal validity; that is, the ability to draw causal conclusions. To complement our work, future research should attempt to address these generalisability concerns by expanding to more ecologically valid scenarios that, for example, examine the verbal patterns and strategies of experienced criminals and involve designs in which interviewees can autonomously decide whether or not to lie, the topic of their report, how and when to deliver their lie during their statements.

Finally, I would be remiss to ignore the problem of replication. Decades of research have failed to provide a reliable method for accurately detecting deception that can be met by the scientific standard of replication. The findings presented in this thesis are not immune to similar replication issues, and it is therefore necessary to perform both direct and conceptual replications before any major conclusions can be appropriately drawn. This is particularly important given the replication crisis that is currently topical in the psychological field. The results of a recent large-scale replication project revealed that only about one third (39\%) of psychological studies that were published in premier journals could replicate (Open Science Collaboration, 2015). The replication crisis is not unique to psychology; one study found that only six of 53 prestigious 
papers in cancer biology could be reproduced (Begley \& Ellis, 2012). However, certain features of psychology, and in particular deception research, incite a particular vulnerability to replicability issues. In his pivotal paper, Luke (2019) provides a critical review of the literature on deception cues. Using statistical simulations, he determined that the scientific study of deception offers very weak evidence for the presence of observable cues between truths and lies. He also concluded that the effect sizes of many deception cues may be greatly overestimated by factors such as low statistical power, few replications, and publication bias. The few cues that appeared to reliably correlate to deception may have actually been a function of sampling error (Luke, 2019). Another interesting observation was that meta-analytic effect sizes for deception cues were negatively related to the number of studies in which that cue had been examined (see Bond, Levine, \& Hartwig, 2014). Moreover, Luke recently wrote a blog post about a similar pattern he observed within studies of the DePaulo et al. (2003) meta-analysis: The more cues a particular study measured, the closer the average of all its cue effects was to zero (Luke, 2020). Taken together, deception researchers are faced with compelling evidence that we have been studying effects that are mostly or entirely statistical noise.

Another explanation for the replication crisis relates to the use of questionable research practices, such as selective reporting and hypothesising after the results are known (the latter colloquially known as HARKing; e.g., Agnoli et al., 2017; Kerr, 1998). Such practices are a problem in psychology generally (e.g., Simmons, Nelson, \& Simonsohn, 2011) but appear particularly common in the deception literature. This could be a result of the high degree of flexibility in coding and analysis (Luke, 2019). The incentives to publish novel, significant results combined with a problematic amount of flexibility may lead researchers towards questionable practices (Nosek, Spies, \& Motyl, 2012). In an effort to combat such practices, two of the three experiments (Chapters 4 and 5) in the current thesis were preregistered via the Open Science Framework and the respective coding schemes were made publically available. The 
published studies (Chapters 2 through 5) are Open Access and the corresponding datasets are also openly available to encourage reproducibility.

\section{Overview of Future Research}

The research presented in this thesis is intended as a starting point, which should be expanded to contexts that more closely resemble real life credibility assessments; for instance, when statements have lies and truths dispersed throughout or during more dynamic interview scenarios. Various concepts could be explored in further studies to help develop our understanding of embedded lies. Some of these future research ideas have been addressed in the current chapter, and others have been provided in the discussion sections of Chapters 2, 3, 4, and 5. Bringing together the findings of this thesis, I will provide several final suggestions for future work on this topic.

The research conducted within this thesis revealed an important finding for deception researchers: Verbal credibility assessment tools that are based on the criteria measured in this research may be robust against the embedding of lies. According to our research findings, it seems that although liars may attempt to strategically maintain consistency in terms of the verbal quality of their truths and lies, their ability to do so may not be strong enough to undermine the theoretical foundations of tools such as CBCA and RM. Additional research is necessary to replicate these results, and should extend these findings by evaluating the full set of CBCA and $\mathrm{RM}$ criteria, by incorporating more realistic crime simulations, and by testing professional lie detectors' ability to distinguish between partially and fully fabricated lies using the complete CBCA and RM protocols.

In this thesis, I have raised the suggestion that deception researchers may have already been examining embedded lies. Underlying most verbal lie detection research is the assumption that lies and truths stem from different production processes (e.g., the theoretical groundwork for CBCA and RM). Instead, lies might be constructed in a process similar to that of truths - by 
drawing on readily accessible information in memory. Reconstructive memory processes are likely activated when people recreate a true experience from long-term (episodic and semantic) memory, as well as when they attempt to invent a story from imagination (Sporer, 2016). Future research should continue exploring the dynamic memory processes involved in generating deceptive accounts, and to what extent these processes correspond to the production of truthful stories. It would be particularly interesting to disentangle how liars draw on their memory traces to produce embedded lies. Insight on this topic could be informative not only for developing ways to exploit such lies, but to also spur new theoretical ideas to account for differences between truths and lies, even if both production processes relate to truthful previous experiences.

Much of the research in this thesis examined detail richness. Deception researchers acknowledge that cues to deception are generally weak but that a handful of cues can significantly distinguish between truthful and deceptive messages (DePaulo et al., 2003; Hartwig \& Bond, 2011). Nonetheless, the estimated effect sizes of deception cues may be artificially inflated by publication bias, low power, and small numbers of estimates (Luke, 2019). One of the only cues to deception that has a reliable body of evidence is the level of detail within a statement (Luke, 2019). Researchers have also reported the level of detail as a significant determinant of veracity when considering partially or fully fabricated statements (Nahari et al., 2012). A general recommendation is for researchers to continue emphasising the level of detail as a cue to deception and to de-emphasise the inclusion of cues that are not supported by well-specified theories (Luke, 2019).

The research conducted in this thesis also exemplified the importance of digging deeper into the strategic embedding of lies for the purposes of lie detection. Being aware of interviewees' strategy use is the first step towards altering and exploiting it. As such, this thesis calls for an increased focus on the development of lie detection methods that aim to exploit interviewees' strategic inclusion of truths and lies within statements. For instance, it is possible that applying 
empirically validated strategy-based techniques to smaller units of analysis within a single statement could be an effective step towards detecting and exploiting embedded lies. Recent research tested the efficacy of a Theme-Selection approach to detecting which part of a statement included a lie, finding that this within-subjects measure yielded stronger results than examining typical between-subjects differences of liars' and truth-tellers' entire interviews (Palena et al., 2019). Similarly, drawing from the computational linguistics field, Bachenko et al. (2008) developed a tagging system that discriminated between deceptive and non-deceptive passages within a written narrative.

Deception researchers should continue focusing their efforts on such developments that target smaller portions within single statements. This could allow for investigators to pinpoint deceptive hotspots within a statement, which would be advantageous for informing where to devote their investigative efforts. First described by Ekman (1985/2009), the term hotspot refers to signs of an emotional or cognitive stressor suggesting possible deception within a certain portion of an individuals' account. Hotspots are typically associated with Ekman's theories of nonverbal emotional leakage (e.g., micro expressions), which have received little, if any, empirical support (e.g., Burgoon, 2018; Vrij, 2018). Although most of Ekman's ideas have been rejected by the majority of the academic community, the core idea of hotspots may still hold value. The recognition of deceptive hotspots, or potential signs of deception within the verbal content of a statement, could encourage the acquisition of additional information (i.e., by further questioning, considering alternative scenarios, or focused investigative efforts to obtain evidence). This approach would reduce investigators' tendency to immediately interpret symptoms of deception as indicative of guilt (Vrij, Granhag, \& Porter, 2010) and instead encourages further investigation and consideration of the context.

A general recommendation, therefore, is for both researchers and practitioners to move away from making global judgements of veracity; that is, deciding if an interviewee in the case 
at hand is deceptive versus honest, or guilty versus innocent. Instead, lie detectors would benefit from a shift towards making continuous judgements of deception, whereby the veracity of individual details is evaluated over the course of a statement (e.g., Leins et al., 2017). This type of evaluation is necessary to reflect the strategic behaviours of liars who interweave truths and lies, as evidenced by the research conducted in this thesis. Deception researchers and practitioners may then be better able to detect when individual parts of a statement are truths and lies.

Not least of all, my final recommendation is for future research to work towards addressing the factors involved in the replication crisis. This crisis will not be resolved by requiring all findings to be replicated prior to publication; such a system would likely do little more than stifle discovery-oriented research (see Lewandowsky \& Oberauer, 2020). Rather, the deception research community can seek to implement a number of methodological reforms, some of which are already popularizing. Luke (2019) offers two recommendations for preventing or reducing the issues relating to lack of replication, low power, and selective reporting. First, researchers are encouraged to increase their transparency in research by fully disclosing their methods, analyses, and data; for instance, via pre-registrations of coding and analysis, publishing openly available data, and adding research to preprint repositories. Second, researchers should aim to improve the statistical power of their work by using, for example, larger sample sizes and/or within-subjects designs. Relatedly, another suggestion is for deception researchers to prioritise the establishment of deception theories (e.g., Nahari et al., 2019). Doing so would help to narrow down which cues are worth examining, help researchers avoid imprecise hypotheses, and aid in the meaningful interpretation of results. Luke's recent (2019) paper casts serious doubt upon the field of deception detection, and the revival of the field may require researchers to take a few steps back, to enable a broader perspective necessary to understand the mechanisms behind 
the phenomenon of deception. Only then should we seek to apply the insights gained in more ecologically valid contexts.

\section{Conclusion}

Across various contexts, from police interviews to legal proceedings and asylum procedures, credibility assessment is fundamental to the administration of justice. For millennia, people have sought ways to separate truths from lies - in medieval times through torture or trials by ordeal (Vicianova, 2015). Modern science, fortunately, has progressed the field of lie detection, although there is still room for improvement. A recent example that highlights the importance of continued scientific pursuits in the field of deception detection is the European Union (EU)'s $€ 4.5$ million investment in an automated border control system (iBorderCtrl, 2016). This project aims to screen incoming travelers at EU borders by using artificial intelligence-based lie detector tests based on the analysis of micro expressions, a controversial method which has no scientific foundation (e.g., Sánchez-Monedero \& Dencik, 2019). The iBorderCtrl project epitomizes how the misuse of deception research can potentially lead to devastating consequences, such as false incrimination and discriminatory effects. As long as fallacies about deception prevail, and pseudo-scientific ideas permeate social, political and legal domains, research on deception and its detection is both relevant and necessary. Deception research functions not merely to advance science, but - in the broad view - to make worthwhile contributions to practical contexts. With more critical reflection and increased research transparency, it is possible that the deception research community can make further strides towards valuable societal contributions.

In sum, across three experiments and one survey, this thesis examined how interviewees' strategic inclusion of truthful and deceptive information within a single account may affect statement quality, and the subsequent effects for methods of verbal lie detection. We found that liars strategically maintain consistency regarding the quality of information provided between 
truths and lies within their statement; however, their attempts at maintaining consistency do not appear to jeopardise the efficacy of several criteria derived from content-based verbal credibility assessment tools. This thesis emphasises that lie detectors should exploit liars' attempts at maintaining consistency by utilising methods of verbal baselining that control for both the individual and the situation. More generally, this thesis suggests that future research should broaden the discourse of deception from a truth-lie dichotomy to instead reflect that the amount of truthful and deceptive information within statements exists on a continuum. 


\section{References}

Agnoli, F., Wicherts, J. M., Veldkamp, C. L., Albiero, P., \& Cubelli, R. (2017). Questionable research practices among Italian research psychologists. PLoS ONE, 12, e0172792. doi:10.1371/journal.pone.0172792

Alison, L., Alison, E., Noone, G., Elntib, S., Waring, S., \& Christiansen, P. (2014). Whatever you say, say nothing: Individual differences in counter interrogation tactics amongst a field sample of right wing, AQ inspired and paramilitary terrorists. Personality and Individual Differences, 68, 170-175. doi: 10.1016/j.paid.2014.04.031

Alonso-Quecuty, M. L. (1992). Deception detection and reality monitoring: a new answer to an old question? In F. Lösel, D. Bender and T. Bliesener (Eds.), Psychology and Law: International Perspectives (pp. 328-332). Berlin: Walter de Gruyter.

Alonso-Quecuty, M. L. (1995). Detecting fact from fallacy in child and adult witness accounts. In G. Davies, S. Lloyd-Bostock, M. McMurran and C. Wilson (Eds.), Psychology, Law and Criminal Justice. International Developments in Research and Practice (pp. 74 80). Berlin: Walter de Gruyter.

Amado, B. G., Arce, R., Fariña, F., \& Vilarino, M. (2016). Criteria-Based Content Analysis (CBCA) reality criteria in adults: A meta-analytic review. International Journal of Clinical and Health Psychology, 16, 201-210. doi: 10.1016/j.ijchp.2016.01.002

Bachenko, J., Fitzpatrick, E., \& Schonwetter, M. (2008). Verification and implementation of language-based deception indicators in civil and criminal narratives. In Proceedings of the 22nd International Conference on Computational Linguistics-Volume 1: 41-48. Association for Computational Linguistics. Retrieved from: http://dl.acm.org/citation.cfm?id=1599087. doi: 10.3115/1599081.1599087

Begley, C. G., \& Ellis, L. M. (2012). Raise standards for preclinical cancer research. Nature, 483, 531-533. 
Bell, K. L., \& DePaulo, B. M. (1996). Liking and lying. Basic and Applied Social Psychology, 18, 243-266. doi:10.1207/s15324834basp1803_1

Blair, J. P., Reimer, T. O., \& Levine, T. R. (2018). The role of consistency in detecting deception: The superiority of correspondence over coherence. Communication Studies, 69, 483-498. doi:10.1080/10510974.2018.1447492

Bogaard, G., Meijer, E. H., \& Van der Plas, I. (2019). A model statement does not enhance the verifiability approach. Applied Cognitive Psychology. doi:10.1002/acp.3596

Bogaard, G., Meijer, E. H., Vrij, A., \& Merckelbach, H. (2016). Strong, but wrong: Lay people's and police officers' beliefs about verbal and nonverbal cues to deception. PLoS ONE, 11, e0156615. doi:10.1371/journal.pone.0156615

Bond, J. C. F., \& DePaulo, B. M. (2006). Accuracy of deception judgments. Personality and Social Psychology Review, 10, 214-234. doi:10.1207/s15327957pspr1003_2

Bond, J. C. F, \& DePaulo, B. M. (2008). Individual differences in judging deception: accuracy and bias. Psychological Bulletin, 134, 477-92. doi: 10.1037/0033-2909.134.4.477 PMID: 18605814

Bond, J. C. F., Kahler, K. N., \& Paolicelli, L. M. (1985). The miscommunication of deception: An adaptive perspective. Journal of Experimental Social Psychology, 21, 331-345. doi:10.1016/0022-1031(85)90034-4

Bond, C. F., Jr., Levine, T. R., \& Hartwig, M. (2014). New findings in non-verbal lie detection. In P. A. Granhag, A. Vrij, \& B. Verschuere (Eds.), Detecting deception: Current challenges and cognitive approaches (pp. 37-58). New York, NY: Wiley

Boskovic, I., Bogaard, G., Merckelbach, H., Vrij, A., \& Hope, L. (2017) The Verifiability Approach to detection of malingered physical symptoms, Psychology, Crime \& Law, 23, 717-729. doi:10.1080/1068316X.2017.1302585 
Brandt, D. R., Miller, G. R., \& Hocking, J. E. (1980a). Effects of self-monitoring and familiarity on deception detection. Communication Quarterly, 28, 3-10.

Brandt, D. R., Miller, G. R., \& Hocking, J. E. (1980b). The truth deception attribution: Effects of familiarity on the ability of observers to detect deception. Human Communication Research, 6, 99-110. doi:10.1111/j.1468-2958.1980.tb00130.x

Brandt, D. R., Miller, G. R., \& Hocking, J. E. (1982). Familiarity and lie detection: A replication and extension. Western Journal of Speech Communication, 46, 276-290. doi:10.1080/10570318209374086

Brown, E. (2018, September 17). California professor, writer of confidential Brett Kavanaugh letter, speaks out about her allegation of sexual assault. Retrieved from https://www.washingtonpost.com/investigations/california-professor-writer-ofconfidential-brett-kavanaugh-letter-speaks-out-about-her-allegation-of-sexualassault/2018/09/16/46982194-b846-11e8-94eb-3bd52dfe917b_story.html

Burgoon, J. K. (2018). Microexpressions are not the best way to catch a liar. Frontiers in psychology, 9, 1672.

Casler, K., Bickel, L., \& Hackett, E. (2013). Separate but equal? A comparison of participants and data gathered via Amazon's MTurk, social media, and face-to-face behavioral testing. Computers in Human Behavior, 29, 2156-2160. doi:10.1016/j.chb.2013.05.009

Caso, L., Palena, N., Carlessi, E., \& Vrij, A. (2019). Police accuracy in truth/lie detection when judging baseline interviews. Psychiatry, Psychology and Law, 1-10. doi: $10.1080 / 13218719.2019 .1642258$

Caso, L., Palena, N., Vrij, A., \& Gnisci, A. (2019). Observers' performance at evaluating truthfulness when provided with comparable truth or small talk baselines. Psychiatry, Psychology and Law, 1-9. doi:10.1080/13218719.2018.1553471 
Chan, D. (2009). So why ask me? Are self-report data really that bad. Statistical and methodological myths and urban legends: Doctrine, verity and fable in the organizational and social sciences, 309-336.

Chandler, J., \& Shapiro, D. (2016). Conducting clinical research using crowdsourced convenience samples. Annual review of clinical psychology, 12.

Clemens, F., Granhag, P. A., \& Strömwall, L. A. (2013). Counter-interrogation strategies when anticipating questions on intentions. Journal of Investigative Psychology and Offender Profiling, 10, 125-138. doi:10.1002/jip.1387

Clemens, F., \& Grolig, T. (2019). Innocent of the crime under investigation: Suspects' counter-interrogation strategies and statement-evidence inconsistency in strategic vs. non-strategic interviews. Psychology, Crime \& Law, 1-35. doi:10.1080/1068316X.2019.1597093

Cohen, J. (1988). Statistical power analysis for the behavioral sciences (2nd ed.). Hillsdale, N.J: L. Erlbaum Associates.

Colwell, K., Hiscock-Anisman, C., Memon, A., Woods, D., \& Michlik, P. M. (2006). Strategies of impression management among deceivers and truth-tellers: How liars attempt to convince. American Journal of Forensic Psychology, 24, 31-38.

Colwell, K., Memon, A., James-Kangal, N., Cole, L., Martin, M., Wirsing, E., \& Cooper, B. (2018). Innocent suspects lying by omission. Journal of Forensic Psychology, 3, 1-6. doi: 10.4172/2475-319X.1000133

Comadena, M. E. (1982). Accuracy in detecting deception: Intimate and friendship relationships. In M. Burgoon (Ed.), Communication yearbook 6 (pp. 446-472). Beverly Hills, CA: Sage.

Craig, D. R. (2003). The right to silence and undercover police operations. International Journal of Police Science \& Management, 5, 112-125. doi:10.1350/ijps.5.2.112.14322 
Dale, D. (2019, June 5). Donald Trump has now said more than 5,000 false things as president. Retrieved from https://www.thestar.com/news/world/analysis/2019/06/05/donaldtrump-has-now-said-more-than-5000-false-claims-as-president.html

Deeb, H., Vrij, A., Hope, L., Mann, S., Granhag, P. A., \& Lancaster, G. L. J. (2017). Suspects' consistency in statements concerning two events when different question formats are used. Journal of Investigative Psychology and Offender Profiling, 14, 74-87. doi:10.1002/jip.1464

Deeb, H., Vrij, A., Hope, L., Mann, S., Granhag, P. A., \& Strömwall, L. A. (2018). Police officers' perceptions of statement inconsistency. Criminal Justice and Behavior, 45, 644-665. doi:10.1177/0093854818758808

DePaulo, B. M. (1992). Nonverbal behavior and self-presentation. Psychological Bulletin, 111, 203-243. doi:10.1037/0033-2909.111.2.203

DePaulo, B. M., \& Kashy, D. A. (1998). Everyday lies in close and casual relationships. Journal of Personality and Social Psychology, 74, 63-79. doi:10.1037/00223514.74.1.63

DePaulo, B. M., Kashy, D. A., Kirkendol, S. E., Wyer, M. M., \& Epstein, J. A. (1996). Lying in everyday life. Journal of Personality and Social Psychology, 70, 979-995. doi:10.1037//0022-3514.70.5.979

DePaulo, B. M., Lindsay, J. J., Malone, B. E., Muhlenbruck, L., Charlton, K., \& Cooper, H. (2003). Cues to deception. Psychological Bulletin, 129, 74-112. doi:10.1037//00332909.129.1.74

DePaulo, B. M., \& Rosenthal, R. (1979). Telling lies. Journal of Personality and Social Psychology, 37, 1713-1722. doi: 10.1037/0022-3514.37.10.1713 
Dilnot, A. (2016). UK Statistics Authority, UK contributions to the European Union. Retrieved from https://www.statisticsauthority.gov.uk/wp-content/uploads/2016/04/Letter-fromSir-Andrew-Dilnot-to-Norman-Lamb-MP-210416.pdf

Dunn, O. J. (1964). Multiple comparisons using rank sums. Technometrics, 6, 241-252. doi:10.1080/00401706.1964.10490181

Ekman, P. (1985/2009). Telling lies: Clues to deceit in the marketplace, politics, and marriage (revised edition). WW Norton \& Company.

Ekman, P., \& Friesen, W. V. (1974). Detecting deception from the body or face. Journal of Personality and Social Psychology, 29, 288-298.

Elntib, S., Wagstaff, G. F., \& Wheatcroft, J. M. (2015). The role of account length in detecting deception in written and orally produced autobiographical accounts using reality monitoring. Journal of Investigative Psychology and Offender Profiling, 12, 185-198. doi:10.1002/jip.1420

Ericsson, K. A., \& Simon, H. A. (1980). Verbal reports as data. Psychological Review, 87, 215251. doi: 10.1037/0033-295X.87.3.215

Estepa, J., \& Kelly, E. (2018, September 28). Brett Kavanaugh: A timeline of allegations against the Supreme Court nominee. Retrieved from http://www.usatoday.com/story/news/politics/onpolitics/2018/09/24/brett-kavanaughallegations-timeline-supreme-court/1408073002/

Evans, J. R., Michael, S. W., Meissner, C. A., \& Brandon, S. E. (2013). Validating a new assessment method for deception detection: Introducing a Psychologically Based Credibility Assessment Tool. Journal of Applied Research in Memory and Cognition, 2, $33-41$. 
Ewens, S., Vrij, A., Jang, M., \& Jo, E. (2014). Drop the small talk when establishing baseline behaviour in interviews. Journal of Investigative Psychology and Offender Profiling, 11, 244-252. doi:10.1002/jip.1414

Feeley, T. H., deTurck, M. A., \& Young, M. J. (1995). Baseline familiarity in lie detection. Communication Research Reports, 12, 160-169. doi:10.1080/08824099509362052

Feitosa, J., Joseph, D. L., \& Newman, D. A. (2015). Crowdsourcing and personality measurement equivalence: A warning about countries whose primary language is not English. Personality and Individual Differences, 75, 47-52. doi:10.1016/j.paid.2014.11.017

Feldman, R. S., Forrest, J. A., \& Happ, B. R. (2002). Self-presentation and verbal deception: Do self-presenters lie more? Basic and Applied Social Psychology, 24, 163-170. doi:10.1207/153248302753674848

Fisher, R. J. (1993). Social desirability bias and the validity of indirect questioning. Journal of Consumer Research, 20, 303-315. doi:10.1086/209351

Fisher, A. J., Medaglia, J. D., \& Jeronimus, B. F. (2018). Lack of group-to-individual generalizability is a threat to human subjects research. Proceedings of the National Academy of Sciences of the United States of America, 115, E6106-E6115. doi:10.1073/pnas.1711978115

Fiske, S. T., \& Taylor, S. E. (2008). Social cognition: From brains to culture. Boston: McGrawHill.

Fleischer, A., Mead, A. D., \& Huang, J. (2015). Inattentive responding in MTurk and other online samples. Industrial and Organizational Psychology, 8, 196-202.

Frank, M. G., Yarbrough, J. D., \& Ekman, P. (2006). Investigative interviewing and the detection of deception. In T. Williamson (Ed.), Investigative interviewing: Rights, research and regulation (pp. 229-255). Devon, United Kingdom: Willan Publishing. 
Funder, D. C., \& Ozer, D. J. (2019). Evaluating effect size in psychological research: Sense and nonsense. Advances in Methods and Practices in Psychological Science. doi:10.1177/2515245919847202

George, J. F., \& Robb, A. (2008). Deception and computer-mediated communication in daily life. Communication Reports, 21, 92-103. doi:10.1080/08934210802298108

Granhag, P. A., Clemens, F., \& Strömwall, L. A. (2009). The usual and the unusual suspects: Level of suspicion and counter-interrogation tactics. Journal of Investigative Psychology and Offender Profiling, 6, 129-137. doi: 10.1002/jip.101

Granhag, P. A., \& Hartwig, M. (2008). A new theoretical perspective on deception detection: On the psychology of instrumental mind-reading. Psychology, Crime \& Law, 14, 189200. doi:10.1080/10683160701645181

Granhag, P. A., \& Strömwall, L. A. (2002). Repeated interrogations: Verbal and non-verbal cues to deception. Applied Cognitive Psychology, 16, 243-257. doi:10.1002/acp.784

Granhag, P. A., Strömwall, L. A., \& Jonsson, A. C. (2003). Partners in crime: How liars in collusion betray themselves. Journal of Applied Social Psychology, 33, 848-868. doi:10.1111/j.1559-1816.2003.tb01928.x

Griesel, D., Ternes, M., Schraml, D., Cooper, B. S., \& Yuille, J. C. (2013). The ABC's of CBCA: Verbal credibility assessment in practice. In Applied issues in investigative interviewing, eyewitness memory, and credibility assessment (pp. 293-323). Springer, New York, NY.

Gnisci, A., Caso, L., \& Vrij, A. (2010). Have you made up your story? The effect of suspicion and liars' strategies on reality monitoring. Applied Cognitive Psychology, 24, 762-773. doi:10.1002acp.1584 
Hafer, C.L., \& Bègue, L. (2005). Experimental research on just world theory: Problems, developments, and future challenges. Psychological Bulletin, 131, 128-167. doi:10.1037/0033-2909.131.1.128

Halevy, R., Shalvi, S., \& Verschuere, B. (2014). Being honest about dishonesty: Correlating self-reports and actual lying. Human Communication Research, 40, 54-72. doi:10.1111/hcre.12019

Hartwig, M., \& Bond, C. F. (2011). Why do lie-catchers fail? A lens model meta-analysis of human lie judgments. Psychological Bulletin, 137, 643-659. doi:10.1037/a0023589

Hartwig, M., Granhag, P. A., \& Strömwall, L. A. (2007). Guilty and innocent suspects' strategies during police interviews. Psychology, Crime and Law, 13, 213-227. doi:10.1080/10683160600750264

Hartwig, M., Granhag, P. A., Strömwall, L. A., \& Doering, N. (2010). Impression and information management: On the strategic self-regulation of innocent and guilty suspects. The Open Criminology Journal, 3, 10-16. doi:10.2174/1874917801003010010.

Hartwig, M., Granhag, P.A., Strömwall, L.A., \& Vrij, A. 2004. Police Officers’ Lie Detection Accuracy: Interrogating Freely versus Observing Video. Police Quarterly, 7, 429-456. doi:10.1177/1098611104264748

Harvey, A. C., Vrij, A., Nahari, G., \& Ludwig, K. (2016). Applying the verifiability approach to insurance claims settings: Exploring the effect of the information protocol. Legal and Criminological Psychology, 22, 47-59. doi: 10.1111/lcrp.12092

Hauch, V., Sporer, S. L., Michael, S. W., \& Meissner, C. A. (2016). Does training improve the detection of deception? A meta-analysis. Communication Research, 43, 283-343. doi:10.1177/0093650214534974 
Hayano, D. M. (1980). Communicative competency among poker players. Journal of Communication, 30, 113-120.

iBorderCtrl. (2016). IBorderCtrl. https://www.iborderctrl.eu/

Inbau, F. E., Reid, J. E., Buckley, J. P., \& Jayne, B. C. (2013). Criminal interrogation and confessions (5th ed.). Burlington, MA: Jones \& Bartlett Learning.

Jarosz, A. F., \& Wiley, J. (2014). What are the odds? A practical guide to computing and reporting Bayes factors. The Journal of Problem Solving, 7, 2-9. doi:10.7771/19326246.1167

Jeffreys, H. (1961). Theory of probability. Oxford, UK: Oxford University Press.

Johnson, M. K. (2006). Memory and reality. American Psychologist, 61, 760-771.

Johnson, M. K., \& Raye, C. L. (1981). Reality monitoring. Psychological Review, 88, 67-85. doi: 10.1037/0033-295X.88.1.67

Johnson, M. K., Bush, J. G., \& Mitchell, K. J. (1998). Interpersonal reality monitoring: Judging the sources of other people's memories. Social Cognition, 16, 199-224. doi:10.1521/ soco.1998.16.2.199

Jundi, S., Vrij, A., Hope, L., Mann, S., \& Hillman, J. (2013). Establishing evidence through undercover and collective intelligence interviewing. Psychology, Public Policy, \& Law, 19, 297-306. doi: 10.1037/a0033571

Kerr, N. L. (1998). HARKing: Hypothesizing after the results are known. Personality and Social Psychology Review, 2, 196-217

Koo, T. K., \& Li, M. Y. (2016). A guideline of selecting and reporting intraclass correlation coefficients for reliability research. Journal of Chiropractic Medicine, 15, 155-163. doi:10.1016/j.jcm.2016.02.012 
Landers, R. N., \& Behrend, T. S. (2015). An inconvenient truth: Arbitrary distinctions between organizational, mechanical turk, and other convenience samples. Industrial and Organizational Psychology, 8, 142-164. doi:10.1017/iop.2015.13

Law, M. K. H., Jackson, S. A., Aidman, E., Geiger, M., Olderbak, S., \& Kleitman, S. (2018). It's the deceiver, not the receiver: No individual differences when detecting deception in a foreign and a native language. PLoS ONE, 13, e0196384. doi:10.1371/journal.pone.0196384

Leal, S., Vrij, A., Deeb, H., \& Jupe, L. (2018). Using the model statement to elicit verbal differences between truth tellers and liars: the benefit of examining core and peripheral details. Journal of Applied Memory and Cognition, 492. doi: 10.1016/j.jarmac.2018.07.001

Leal, S., Vrij, A., Nahari, G., \& Mann, S. (2016). Please be honest and provide evidence: Deterrents of deception in an online insurance fraud context. Applied Cognitive Psychology, 30, 768-774. doi:10.1002/acp.3252

Lee, M. D., \& Wagenmakers, E. J. (2013). Bayesian cognitive modeling: A practical course. Cambridge, New York: Cambridge University Press.

Leins, D., Fisher, R. P., \& Ross, S. J. (2013). Exploring liars' strategies for creating deceptive reports. Legal and Criminological Psychology, 18, 141-151. doi:10.1111/j.20448333.2011.02041.x

Leins, D. A., Fisher, R. P., \& Vrij, A. (2012). Drawing on liars' lack of cognitive flexibility: Detecting deception through varying report modes. Applied Cognitive Psychology, 26, 601-607. doi:10.1002/acp.2837

Leins, D., Fisher, R. P., Vrij, A., Leal, S., \& Mann, S. (2011). Using sketch drawing to induce inconsistency in liars. Legal and Criminological Psychology, 16, 253-265. 
Leins, D. A., Zimmerman, L. A., \& Polander, E. N. (2017). Observers' real-time sensitivity to deception in naturalistic interviews. Journal of Police and Criminal Psychology, 32, 319-330. doi:10.1007/s11896-017-9224-2

Levine, T. R. (2010). A few transparent liars explaining 54\% accuracy in deception detection experiments. Annals of the International Communication Association, 34, 41-61. doi:10.1080/23808985.2010.11679095

Levine, T. R. (2016). Examining sender and judge variability in honesty assessments and deception detection accuracy: Evidence for a transparent liar but no evidence of deception-general ability. Communication Research Reports, 33, 188-194. doi:10.1080/08824096.2016.1186629

Levine, T. R., Serota, K. B., Shulman, H., Clare, D. D., Park, H. S., Shaw, A. S., . . Lee, J. H. (2011). Sender demeanor: Individual differences in sender believability have a powerful impact on deception detection judgments. Human Communication Research, 37, 377403. doi:10.1111/j.1468-2958.2011.01407.x

Lewandowsky, S., \& Oberauer, K. (2020). Low replicability can support robust and efficient science. Nature Communications, 11, 1-12.

Lichfield, J. (2017, September 18). Boris Johnson's £350m claim is devious and bogus. Here's why. Retrieved from https://www.theguardian.com/commentisfree/2017/sep/18/borisjohnson-350-million-claim-bogus-foreign-secretary

Luke, T. J. (2019). Lessons from pinocchio: Cues to deception may be highly exaggerated. Perspectives on Psychological Science, 14, 646-671. doi:10.1177/1745691619838258

Luke, T. J. (2020, February 3). Yet another decline effect in the deception cue literature. Retrieved from https://www.rabbitsnore.com/2020/02/yet-another-decline-effect-indeception.html 
Luke, T. J., Hartwig, M., Joseph, E., Brimbal, L., Chan, G., Dawson, E., . . Granhag, P. A. (2016). Training in the strategic use of evidence technique: Improving deception detection accuracy of American law enforcement officers. Journal of Police and Criminal Psychology, 31, 270-278. doi:10.1007/s11896-015-9187-0

Mann, S., Vrij, A., \& Bull, R. (2004). Detecting true lies: Police officers' ability to detect suspects' lies. Journal of Applied Psychology, 89, 137-149. doi:10.1037/00219010.89 .1 .137

Masip, J., \& Herrero, C. (2013). 'What would you say if you were guilty?' Suspects' strategies during a hypothetical behavior analysis interview concerning a serious crime. Applied Cognitive Psychology, 27, 60-70. doi: 10.1002/acp.2872

Masip, J., Sporer, S. L., Garrido, E., \& Herrero, C. (2005). The detection of deception with the reality monitoring approach: A review of the empirical evidence. Psychology, Crime \& Law, 11, 99-122. doi: 10.1080/10683160410001726356

McCornack, S. A., Morrison, K., Paik, J. E., Wisner, A. M., \& Zhu, X. (2014). Information manipulation theory 2: A propositional theory of deceptive discourse production. Journal of Language and Social Psychology, 33, 348-377. doi:10.1177/0261927X14534656

McComack, S. A., \& Parks, M. R. (1986). Deception detection and relational development: The other side of trust. In M. L. McLaughlin (Ed.), Communication yearbook 9 (pp. 377389). Beverly Hills, CA: Sage.

Meade, A. W., \& Craig, S. B. (2012). Identifying careless responses in survey data. Psychological Methods, 17, 437-455. doi:10.1037/a0028085

Meissner, C. A., \& Kassin, S. M. (2002). “He’s guilty!” Investigator bias in judgments of truth and deception. Law and Human Behavior, 26, 469-480. doi:

10.1023/A:1020278620751 
Meixner, J. B., \& Rosenfeld, J. P. (2014). Detecting knowledge of incidentally acquired, realworld memories using a P300-based concealed-information test. Psychological science, 25, 1994-2005. doi: 10.1177/0956797614547278

Merckelbach, H. (2004). Telling a good story: fantasy proneness and the quality of fabricated memories. Personality and Individual Differences, 37, 1371-1382. doi: 10.1016/j.paid.2004.01.007 54

Mohamed, F. B., Faro, S. H., Gordon, N. J., Platek, S. M., Ahmad, H., \& Williams, J. M. (2006). Brain mapping of deception and truth telling about an ecologically valid situation: Functional MR imaging and polygraph investigation-initial experience. Radiology, 238, 679.

Morrissey, E. R. (1974). Sources of error in the coding of questionnaire data. Sociological Methods \& Research, 3, 209-232.

Münsterberg, H. (1908). On the witness stand: Essays on psychology and crime. New York: The McClure Company.

Nahari, G. (2016). When the long road is the shortcut: A comparison between two coding methods for content-based lie detection tools. Psychology, Crime and Law, 22, 1000 1014.

Nahari, G. (2018a). Reality monitoring in the forensic context: Digging deeper into the speech of liars. Journal of Applied Research in Memory and Cognition, 7, 432-440. doi:10.1016/j.jarmac.2018.04.003

Nahari, G. (2018b). The applicability of the verifiability approach to the real world. In P. Rosenfeld (Ed.), Detecting concealed information and deception: Verbal, behavioral, and biological methods (pp. 329-350). San Diego, CA: Academic Press. doi: 10.1016/B978-0-12- 812729-2.00014-8 
Nahari, G., Ashkenazi, T., Fisher, R. P., Granhag, P. A., Hershkowitz, I., Masip, J., ... \& Verschuere, B. (2019). 'Language of lies': Urgent issues and prospects in verbal lie detection research. Legal and Criminological Psychology, 24, 1-23.

Nahari, G. \& Pazuelo, M. (2015). Telling a convincing story: richness in detail as a function of gender and priming. Journal of Applied Research in Memory and Cognition, 4, $363-$ 367. doi: 10.1016/j.jarmac.2015.08.005 55

Nahari, G. \& Vrij, A. (2015). Systematic errors (biases) in applying verbal lie detection tools: Richness in detail as a test case. Crime Psychology Review, 1, 98-107. doi:10.1080/23744006.2016.1158509

Nahari, G., Vrij, A., \& Fisher, R. P. (2012). Does the truth come out in the writing? SCAN as a lie detection tool. Law and Human Behavior, 36, 68-76. doi:10.1037/h0093965

Nahari, G., Vrij, A., \& Fisher, R. P. (2014a). Exploiting liar's verbal strategies by examining the verifiability of details. Legal and Criminological Psychology, 19, 227-239. doi:10.1111/j.2044-8333.2012.02069.x

Nahari, G., Vrij, A., \& Fisher, R. P. (2014b). The verifiability approach: Countermeasures facilitate its ability to discriminate between truths and lies, countermeasures facilitate its ability to discriminate between truths and lies. Applied Cognitive Psychology, 28, 122128. doi: $10.1002 / \mathrm{acp} .2974$

Nosek, B. A., Spies, J. R., \& Motyl, M. (2012). Scientific utopia: II. Restructuring incentives and practices to promote truth over publishability. Perspectives on Psychological Science, 7, 615-631.

Oberlader, V. A., Naefgen, C., Koppehele-Gossel, J., Quinten, L., Banse, R., \& Schmidt, A. F. (2016). Validity of content-based techniques to distinguish true and fabricated statements: A meta-analysis. Law and Human Behavior, 40, 440-457. doi:10.1037/lhb0000193 
Open Science Collaboration. (2015). Estimating the reproducibility of psychological science. Science, 349, aac4716.

Palena, N., Caso, L., \& Vrij, A. (2019). Detecting lies via a theme-selection strategy. Frontiers in Psychology, 9. doi: 10.3389/fpsyg.2018.02775

Palena, N., Caso, L., Vrij, A., \& Orthey, R. (2018). Detecting deception through small talk and comparable truth baselines. Journal of Investigative Psychology and Offender Profiling, 15. 124-132. doi: 10.1002/jip.1495

Porter, S., \& Yuille, J. C. (1996). The language of deceit: An investigation of the verbal clues to deception in the interrogation context. Law and Human Behavior, 20, 443-458. doi:10.1007/BF01498980

Richard, F. D., Bond, C. F., \& Stokes-Zoota, J. J. (2003). One hundred years of social psychology quantitatively described. Review of General Psychology, 7, 331-363. doi:10.1037/1089-2680.7.4.331

Riggio, R. E., Tucker, J., and Throckmorton, B. (1987). Social skills and deception ability. Journal of Personality and Social Psychology, 13, 568-577. doi: $10.1177 / 0146167287134013$

Rizzo, S., Kessler, G., \& Kelly, M. (2019, December 16). President Trump has made 15,413 false or misleading claims over 1,055 days. Retrieved from https://www.washingtonpost.com/politics/2019/12/16/president-trump-has-made-falseor-misleading-claims-over-days/

Ruby, C. L., \& Brigham, J. C. (1998). Can Criteria-Based Content Analysis distinguish between true and false statements of African-American speakers? Law and Human Behavior, 22, 369-388. doi: 10.1023/a:1025766825429 
Russano, M. B., Narchet, F. M., Kleinman, S. M., \& Meissner, C. A. (2014). Structured interviews of experienced HUMINT interviewers. Applied Cognitive Psychology, 28, 847-859. doi: 10.1002/acp.3069

Sánchez-Monedero, J., \& Dencik, L. (2019). The politics of deceptive borders:' biomarkers of deceit' and the case of iBorderCtrl. arXiv preprint arXiv:1911.09156.

Schank, R. C., \& Abelson, R. P. (1977). Scripts, plans, goals, and understanding: An inquiry into human knowledge structures. New York; Hillsdale, N.J: L. Erlbaum Associates.

Serota, K. B., \& Levine, T. R. (2015). A few prolific liars: Variation in the prevalence of lying. Journal of Language and Social Psychology, 34, 138-157. doi:10.1177/0261927X14528804

Serota, K. B., Levine, T. R., \& Boster, F. J. (2010). The prevalence of lying in America: Three studies of self-reported lies. Human Communication Research, 36, 2-25. doi:10.1111/j.1468-2958.2009.01366.x

Simmons, J. P., Nelson, L. D., \& Simonsohn, U. (2011). False-positive psychology: Undisclosed flexibility in data collection and analysis allows presenting anything as significant. Psychological Science, 22, 1359-1366.

Sporer, S. L. (2016). Deception and cognitive load: Expanding our horizon with a working memory model. Frontiers in Psychology, 7, 420. doi: 10.3389/fpsyg.2016.00420

Sporer, S. L. and Küpper, B. (1995). Realitätsüberwachung und die Beurteilung des Wahrheitsgehaltes von Erzählungen: Eine experimentelle Studie [Reality monitoring and the judgment of credibility of stories: an experimental study]. Zeitschrift fur Sozialpsychologie, 26, 173-193.

Sporer, S. L., \& Sharman, S. J. (2006). Should I believe this? Reality monitoring of accounts of self-experienced and invented recent and distant autobiographical events. Applied Cognitive Psychology, 20, 837-854. doi: 10.1002/acp.1234 
Street, C. N. H., \& Richardson, D. C. (2015). Lies, damn lies, and expectations: How base rates inform Lie-Truth judgments. Applied Cognitive Psychology, 29, 149-155. doi:10.1002/acp.3085

Steller, M., \& Köhnken, G. (1989). Criteria-based content analysis. In Raskin, D. C. (Ed.), Psychological methods in criminal investigation and evidence (pp. 217-245). New York: Springer Publishing Company.

Strömwall, L. A., Hartwig, M., \& Granhag, P. A. (2006). To act truthfully: Nonverbal behavior and strategies during a police interrogation. Psychology, Crime \& Law, 12, 207-219. doi: 10.1080/10683160512331331328

Strömwall, L., \& Granhag, P. A. (2003). How to detect deception? Arresting the beliefs of police officers, prosecutors and judges. Psychology, Crime \& Law, 9, 19-36. doi:10.1080/10683160308138

Strömwall, L. A., Granhag, P. A., \& Hartwig, M. (2004). Practicioners' beliefs about deception. In L. A. Strömwall \& P. A. Granhag (Eds.), The detection of deception in forensic contexts. (pp. 229-250). Cambridge, England: Cambridge University Press.

Strömwall, L. A., Willén, R. M. (2011). Inside criminal minds: Offenders' strategies when lying. Journal of Investigative Psychology and Offender Profiling, 8, 271-281. doi:10.1002/jip.148

Syed, M., \& Nelson, S. C. (2015). Guidelines for establishing reliability when coding narrative data. Emerging Adulthood, 3, 375-387.

Thalheimer, W., \& Cook, S. (2002, August). How to calculate effect sizes from published research articles: A simplified methodology. Retrieved May 22, 2019 from http://www.bwgriffin.com/gsu/courses/edur9131/content/ Effect_Sizes_pdf5.pdf.

Undeutsch, U. (1967). Beurteilung der glaubhaftigkeit von aussagen [Evaluation of statement credibility/ Statement validity assessment]. In U. Undeutsch (Ed.), Hand-buch der 
Psychologie (Vol. 11: Forensische Psychologie, pp. 26-181). Göttingen, Germany: Hogrefe.

Undeutsch, U. (1989). The development of statement reality analysis. In J. Yuille (Ed.), Credibility assessment (pp.101-119). Dordrech, Holland: Kluwer Academic Publishers.

U.S. Department of the Army (2006). Field manual 2-22.3 (FM 34-52) Human intelligence collector operations. Washington, DC: Headquarters, Department of the Army.

Valois, R., Akehurst, L., Hanway, P., \& Williams, R. (2019). Half truths and whole lies: Credibility judgements of partial and complete fabrications using Criteria-Based Content Analysis and Reality Monitoring. Manuscript submitted for publication.

Van Swol, L. M., \& Braun, M. T. (2014). Communicating deception: Differences in language use, justifications, and questions for lies, omissions, and truths. Group Decision and Negotiation, 23, 1343-1367. doi:10.1007/s10726-013-9373-3

Van Swol, L. M., Braun, M. T., \& Kolb, M. R. (2015). Deception, detection, demeanor, and truth bias in face-to-face and computer-mediated communication. Communication Research, 42, 1116-1142. doi:10.1177/0093650213485785

Verigin, B. L., Meijer, E. H., \& Vrij, A. Embedding lies into truthful stories does not affect their quality. Applied Cognitive Psychology, 34, 516-525. doi: 10.1002/acp.3642

Verigin, B. L., Meijer, E. H., Vrij, A., \& Zauzig, L. (2019). The interaction of truthful and deceptive information. Psychology, Crime and Law, 26, 367-383. doi: 10.1080/1068316X.2019.1669596

Verschuere, B., Vrij, A., \& Granhag, P. A. (2015). Detecting deception: Current challenges and cognitive approaches. Hoboken: Wiley-Blackwell.

Vicianova, M. (2015). Historical techniques of lie detection. Europe's journal of psychology, 11, 522-534. doi:10.5964/ejop.v11i3.919 
Volbert, R., \& Steller, M. (2014). Is this testimony truthful, fabricated, or based on false memory? Credibility assessment 25 years after Steller and Köhnken (1989). European Psychologist, 19, 207-220. doi: 10.1027/1016-9040/a000200

Vredeveldt A., van Koppen P., Granhag P.A. (2014). The Inconsistent Suspect: A Systematic Review of Different Types of Consistency in Truth Tellers and Liars. In: Bull R. (eds) Investigative Interviewing (pp. 189-207). Springer, New York, NY.

Vrij, A. (2005). Criteria-based content analysis: A qualitative review of the first 37 studies. Psychology, Public Policy, and Law, 11, 3-41. https:// doi.org/10.1037/10768971.11.1.3

Vrij, A. (2007). Deception: a social lubricant and a selfish act. In K. Fiedler (Ed.), Social communication (pp. 309-342). (Frontiers of social psychology). New York: Psychology Press.

Vrij, A. (2008a). Detecting lies and deceit: Pitfalls and opportunities, second edition. Chichester: John Wiley and Sons.

Vrij, A. (2008b). Nonverbal dominance versus verbal accuracy in lie detection: A plea to change police practice. Criminal Justice and Behavior, 35, 1323-1336. doi:10.1177/0093854808321530

Vrij, A. (2016). Baselining as a lie detection method. Applied Cognitive Psychology, 30, 11121119. doi:10.1002/acp.3288

Vrij, A. (2018). Deception and truth detection when analyzing nonverbal and verbal cues. Applied Cognitive Psychology, 1-8. doi:10.1002/acp.3457

Vrij, A., Akehurst, L., Soukara, S., \& Bull. R. (2002). Will the truth come out? The effect of deception, age, status, coaching, and social skills on CBCA scores. Law and Human Behaviour, 26, 261-283. doi: 10.1023/A:1015313120905 
Vrij, A., Edward, K., Roberts, K. P., \& Bull, R. (2000). Detecting deceit via analysis of verbal and nonverbal behavior. Journal of Nonverbal Behavior, 24, 239-263. doi:10.1023/A:1006610329284

Vrij, A., Fisher, R. P., \& Blank, H. (2017). A cognitive approach to lie detection: A metaanalysis. Legal and Criminological Psychology, 22, 1-21. doi:10.1111/lcrp.12088

Vrij, A., Granhag, P. A., \& Mann, S. (2010). Good liars. The Journal of Psychiatry \& Law, 38, 77-98. doi:10.1177/009318531003800105

Vrij, A., Granhag, P. A., Mann, S., \& Leal, S. (2011). Outsmarting the liars: Toward a cognitive lie detection approach. Current Directions in Psychological Science, 20, 2832. doi:10.1177/0963721410391245

Vrij, A., Granhag, P. A., \& Porter, S. (2010). Pitfalls and opportunities in verbal and nonverbal lie detection. Psychological Science in the Public Interest, 11, 89-121. doi: $10.1177 / 1529100610390861$

Vrij, A., Leal, S., \& Fisher, R. P. (2018). Verbal deception and the model statement as a lie detection tool. Frontiers in Psychiatry, 9, 492-492. doi:10.3389/fpsyt.2018.00492

Vrij, A., Leal, S., Fisher, R. P., Mann, S., Dalton, G., Jo, E., ... Houston, K. (2018). Sketching as a technique to elicit information and cues to deceit in interpreter-based interviews. Journal of Applied Research in Memory and Cognition, 7, 303-313. doi: 10.1016/j. jrarmac.2017.11.001

Vrij, A., Leal, S., Jupe, L., \& Harvey, A. (2018). Within-subjects verbal lie detection measures: A comparison between total detail and proportion of complications. Legal and Criminological Psychology, 23, 265-279. doi:10.1111/lcrp.12126

Vrij, A., Leal, S., Mann, S., Dalton, G., Jo, E., Shaboltas, A., ... Houston, K. (2017). Using the model statement to elicit information and cues to deceit in interpreter-based interviews. Acta Psychologica, 177, 44-53. doi: 10.1016/j.actpsy.2017.04.011 
Vrij, A., Leal, S., Mann, S., \& Fisher, R. (2012). Imposing cognitive load to elicit cues to deceit: Inducing the reverse order technique naturally. Psychology, Crime \& Law, 18, 579-594. doi:10.1080/ 1068316X2010.515987

Vrij, A., Leal, S., Mann, S., Fisher, R. P., Dalton, G., Jo, E., ... Houston, K. (2018). Using unexpected questions to elicit information and cues to deceit in interpreter-based interviews. Applied Cognitive Psychology, 32, 94-104. doi: 10.1002/acp.3382

Vrij, A., \& Mann, S. (2001). Telling and detecting lies in a high-stake situation: The case of a convicted murderer. Applied Cognitive Psychology, 15, 187-203. doi:10.1002/10990720(200103/04)15:2<187::AID-ACP696>3.0.CO;2-A

Vrij, A., Mann, S., Jundi, S., Hillman, J. \& Hope, L. (2014). Detection of concealment in an information-gathering interview. Applied Cognitive Psychology, 28, 86-866. doi: 10.1002/acp.305

Wagenmakers, E. J., Love, J., Marsman, M., Jamil, T., Ly, A., Verhagen, J., . . Morey, R. D. (2016). Bayesian inference for psychology. Part II: Example applications with JASP, 126. Retrieved from http://maartenmarsman.com/wpcontent/uploads/2017/04/ WagenmakersEtAlPartII.pdf

Walczyk, J. J., Harris, L. L., Duck, T. K., \& Mulay, D. (2014). A social-cognitive framework for understanding serious lies: Activation-decision-construction-action theory. New Ideas in Psychology, 34, 22-36. doi:10.1016/j.newideapsych.2014.03.001

Whitty, M. T., \& Carville, S. E. (2008). Would I lie to you? Self-serving lies and other oriented lies told across different media. Computers in Human Behavior, 24, 1021-1031. doi: 10.1016/j. chb.2007.03.004

Williams, S. (1998). Invisible darkness: the strange case of Paul Bernardo and Karla Homolka. New York: Bantam Books. 
Wright, G. R. T., Berry, C. J., \& Bird, G. (2012). "You can't kid a kidder": Association between production and detection of deception in an interactive deception task. Frontiers in Human Neuroscience, 6, 87. doi: 10.3389/fnhum.2012.00087

Wright, G. R. T., Berry, C. J., \& Bird, G. (2013). Deceptively simple ... the "deceptiongeneral" ability and the need to put the liar under the spotlight. Frontiers in Neuroscience, 7, 152. doi: 10.3389/fnins.2013.00152

Wright, C., \& Wheatcroft, J. M. (2017). Police officers' beliefs about, and use of, cues to deception. Journal of Investigative Psychology and Offender Profiling, 14, 307-319. doi:10.1002/jip.1478

Zhou, L., Burgoon, J.K., Nunamaker, J.F., \& Twitchell, D. (2004). Automatic linguistic-based cues for detecting deception in text-bases asynchronous computer-mediated communication. Group Decision and Negotiation, 13, 81-106.

doi:10.1023/B:GRUP.0000011944.62889.6f 


\section{Summary}

Embedded lies, the topic of investigation in this thesis, have been under researched within the deception detection literature. The overarching aim of this research was to examine how interviewees' strategic inclusion of both truths and lies within a single account may affect statement quality, and the subsequent implications for verbal lie detection.

Across three experimental studies and one survey, we (i) explored the deception strategies reported by people who claim to be good liars (Chapter 2), (ii) examined how the combination of truths and lies within a single account affects the verbal quality of statements (Chapters 3 and 4), and (iii) tested if embedded lies can be exploited to facilitate withinstatement lie detection (Chapter 5). Chapter $2(n=194)$ explored deception by investigating how laypeople's self-reported ability to deceive was associated with their lie frequency, characteristics, and deception strategies. Of particular interest was whether the strategic inclusion of truthful details into lies corresponded to higher perceptions of deception ability. We found that self-reported good liars reported embedding lies as their most common strategy for deceiving successfully. In Chapter 3 ( $n=144)$, we experimentally tested how interviewees strategically regulate the information they provide when their accounts contain both truthful and deceptive information. We found that interviewees calibrated the richness of detail provided in the first element of their statement to be consistent with the veracity of the second element, such that elements followed by a lie were less detailed than elements followed by a truth. Further examination revealed that participants also calibrated their lies according to both the preceding and the following element, with lies becoming more detailed when they were flanked by truthful information compared to when they were flanked by other lies. In light of these results, Chapter $4(n=111)$ examined whether embedded lies differed qualitatively from full fabrications. Our results showed that the two types of lies did not differ on a number of content-based cues (e.g., detail richness), but that lies embedded in otherwise truthful 
statements could be distinguished from truths embedded in truthful statements on the basis of detail richness and statement quality (i.e., clarity and plausibility). Finally, Chapter 5 ( $n=148$ ) tested whether lie detection accuracy could be improved by using embedded lies as a withinstatement baseline comparison. Contrary to our hypothesis, results showed that instructing participants to make a within-statement baseline comparison did not increase the accuracy of their veracity assessments.

Taken together, liars aim to strategically maintain consistency regarding the quality of information provided between truths and lies within their statements. However, content-based methods of verbal credibility assessment based on the cues measured in this thesis may be robust against this strategy. The results of this thesis emphasise that lie detectors should exploit liars' attempts at maintaining consistency by utilising methods of verbal baselining that control for both the individual and the situation. 


\section{Acknowledgements}

It is not easy to articulate how profoundly this experience has changed my life. Achieving a doctorate degree is a significant milestone, and completing it abroad was an experience in its own right. This chapter of my life would not have been possible without the support and inspiration that I received from a number of people, to whom I owe many thanks.

I would first like to thank my supervisor, Ewout. Without your valuable feedback, this $\mathrm{PhD}$ would hardly have been achievable. Thank you for taking a chance on me and for patiently letting me grow into an independent researcher. I have learnt a great deal from you, both about science and integrity. I hope to have picked up at least a fraction of your brilliance; I'd consider myself lucky to have done so. My sincere thanks also goes to my second supervisor, Aldert, whose expertise and (quick!) feedback always improved my work.

I gratefully acknowledge the funding received for my doctoral studies from the Erasmus Mundus House of Legal Psychology programme. My experience in the programme exceeded my expectations in every possible way, and frankly, I am still in awe at the opportunity. Thank you to Peter and everyone involved with bringing this program to fruition, your efforts have truly changed all of our lives. A big thanks also goes to Chantal, who has always been so caring and helpful.

To everyone apart of our EMJD family - through the trials of this thesis you have been a source of motivation and support. I consider myself exceptionally lucky to have been surrounded by academics of your caliber. I'm already looking forward to our reunion; until then, I wish you all much happiness and success. I am especially grateful to Ale, for your friendship and support. You are a wonderful friend and a gifted researcher, and your resilience is inspiring. To Sergii, your kind-heart, sense of humor, and brilliant mind have enriched my life. Thanks for your friendship and support. To Nina and Irena, the 'older siblings' here in Maastricht, thanks for your friendship, guidance, and encouragement. We'd have been lost 
without you. To Renan and the Portsmouth crew, thanks for welcoming us to England and for all of the great memories. I also owe thanks to all of my other PhD friends: Joe, Enide, Carey, Jenn, Bruna, Robin, Sanne and Glynis, for the support and the many entertaining moments.

I am ever grateful to my best friends who have become family to me. Kathy, my other half, you have been by my side since day one of this journey, in both the moments of deep anxiety (read: imposter syndrome) and of big excitement. Thank you for your endless support, for always keeping me laughing, and for being the best travel buddy. There are no proper words to convey my deep gratitude and appreciation for you. Your friendship made all the difference in what would have been a tremendously solitary experience. Suffice it to say that I feel incredibly lucky to have met you and I can't wait for many more adventures together! Michelle, you have never ceased to inspire me, even from across the world. You have taught me so much about strength, resilience, passion, and sense of self. Without you, I would not have had the courage to embark on this journey in the first place. To say thanks seems hardly enough.

I am so deeply grateful to Caleb, who has been a constant source of joy and inspiration for me. Thank you for always celebrating my small victories and for helping me back on my feet during the tough times. Most of all, thank you for your love, for the adventures, and for becoming my home away from home. Meeting you has been the highlight of my time abroad and I am grateful to call this our beginning. I love you.

Finally, this thesis is dedicated to my parents, Sharel and Lorne, without whom this never would have been possible. Your love and encouragement has been unwavering despite the long distance between us. You are my inspiration for this achievement and I will never take that for granted. You've taught me many things, but most importantly, to live a life filled with kindness, a good attitude, a strong work ethic and a spirit of adventure. I also dedicate this thesis to my sister Whitney, who convinced me to take this leap of faith and who has always being my biggest inspiration and supporter. 


\section{Dissemination}

\section{Peer-Reviewed Publications}

Verigin, B. L., Meijer, E. H., Vrij, A., \& Zauzig, L. (2019). The interaction of truthful and deceptive information. Psychology, Crime and Law, 26, 367-383. doi: 10.1080/1068316X.2019.1669596

Verigin, B. L., Meijer, E. H., Bogaard, G., \& Vrij, A. (2019). Lie prevalence, lie characteristics and strategies of self-reported good liars. PLOS ONE, 14, e0225566, doi: 10.1371/journal.pone.0225566

Verigin, B. L., Meijer, E. H., \& Vrij, A. (2020). Embedding lies into truthful stories does not affect their quality. Applied Cognitive Psychology, 34, 516-525. doi: $10.1002 /$ acp.3642

Verigin, B. L., Meijer, E. H., \& Vrij, A. (2020). A within-statement baseline comparison for detecting lies. Psychiatry, Psychology and Law. doi: $10.1080 / 13218719.2020 .1767712$

\section{Conference Oral Presentations}

Verigin, B. L., Meijer, E. H., \& Vrij, A. (July 2019). A within-statement baseline comparison for detecting lies. Presented orally at the European Association of Legal Psychology conference (EAPL), Santiago de Compostela, Spain.

Verigin, B. L., Meijer, E. H., \& Vrij, A. (March 2019). Embedding lies into truthful stories does not affect their quality. Presented orally at the Conference of the American Psychology-Law Society (APLS), Portland, Oregon.

Verigin, B. L., Meijer, E. H., \& Vrij, A. (June 2018). The interaction of truths and lies within statements. Presented orally at the European Association of Legal Psychology conference (EAPL), Turku, Finland.

Verigin, B. L., Meijer, E. H., Vrij, A., \& Merckelbach, H. (May 2017). Where the truth lies: Within-statement verbal lie detection. Presented orally at the European Association of Legal Psychology conference (EAPL), Mechelen, Belgium. 


\section{Curriculum Vitae}

Brianna Leigh Verigin was born on November $1^{\text {st }}, 1994$ in Cranbrook, British Columbia (Canada). In 2012, she graduated from Kelowna Secondary School, after which she started studying psychology at the University of British Columbia-Okanagan. During her studies, Brianna specialized in Forensic Psychology and she completed an Honours thesis on the expression of remorse as a function of psychopathy within a sample of federally incarnated offenders. As part of this specialization, Brianna also completed two fieldwork practicums, with the Royal Canadian Mounted Police (General Investigations Unit) and with the Ministry of Children \& Family Development (Youth Probation). Brianna graduated in June of 2016 as the valedictorian of the Bachelor of Arts class. In September 2016, she began the House of Legal Psychology Erasmus Mundus Joint Doctorate program. Her PhD project on embedded lies and verbal credibility assessment was supervised by Dr. Ewout Meijer in Maastricht, the Netherlands and Prof. dr. Aldert Vrij at the University of Portsmouth, England, where she also spent six months in the Department of Psychology. In November 2019, Brianna successfully defended her doctoral thesis at the University of Portsmouth. Brianna's career aspiration is to conduct research in an applied context, contributing to evidence-based and intelligence-led policing and policy development. 


\section{Valorisation Addendum}

This valorisation chapter addresses the utilisation of scientific knowledge in practice. Besides theoretical relevance, the research described in this thesis also offers practical applications. I have already discussed many of these practical applications in various chapters. Nonetheless, I will highlight several key aspects of knowledge valorisation in this chapter, pertaining to (i) the societal relevance of this research, (ii) the target groups to whom this research concerns, (iii) the activities and services that this research may influence, (iv) the innovative nature of this research, and (v) the implementation and dissemination of these research findings.

\section{Relevance of the Research}

Credibility assessment is fundamental to the administration of social and legal justice. For instance, police and legal actors often encounter deceptive or misleading statements from suspects, witnesses, and even victims. Conducting verbal credibility assessment is of particular importance during investigations or legal proceedings in which physical evidence is absent or inconclusive (e.g., Griesel et al., 2013). For example, when US Supreme Court nominee Brett Kavanaugh was accused of historical sexual abuse, the Senate Judiciary Committee arranged a hearing to determine the veracity of the allegations. The Committee was required to form their assessment largely based on the statements made by Kavanaugh and the woman who raised the allegations (Brown, 2018; Estepa \& Kelly, 2018). This example illustrates the challenge faced by many legal actors who need to determine the credibility of a suspect or witness solely on the basis of statement evidence.

The assessment of credibility is further complicated by the general inability of both laypeople and legal professionals to detect deception. Decades of research show that lie detection accuracy rates barely exceed chance level (Bond \& DePaulo, 2006; Luke et al., 2016; Vrij, 2008a). Accordingly, researchers have invested substantial efforts in developing tools and 
techniques for improving these accuracy rates. CBCA and RM are considered favourable tools among researchers and practitioners since, with appropriate training, evaluators' accuracy rates increase from chance level to $65 \%$ - 80\% (Vrij, 2008a). Still, even under favourable conditions these tools have a high margin of error, which leaves much room for improvement. This research examined how interviewees' strategic inclusion of both truths and lies within a single account may affect statement quality, and the subsequent implications for verbal lie detection.

\section{Target Group}

Outside of academic circles, the results of this thesis could be of interest to police and intelligence agencies, legal practitioners (e.g., judges, lawyers, or expert witnesses), and policy makers. These groups are concerned with evaluating the credibility and investigative value of statements. For instance, psychologists and expert witnesses in the Netherlands, Germany, and the UK reportedly use CBCA to determine the veracity of allegations of child sexual abuse (Oberlader et al., 2016). These target groups may benefit from empirical efforts to determine the reliability of verbal credibility assessment tools and techniques. They may further benefit from knowledge regarding the strategies used by liars and the factors that potentially jeopardise the effectiveness of such tools. Practitioners may find the results of this thesis most informative for circumstances in which they are conducting or evaluating credibility assessments in the absence of physical evidence.

I would like to emphasize that the findings reported in this thesis require additional research and replication before robust recommendations can be offered to practitioners working in applied legal contexts.

\section{Activities and Services}

The results of this thesis suggest that verbal credibility assessment tools that are based on the criteria measured in this research may be robust against the embedding of lies. It follows that practitioners can cautiously proceed with using these tools as investigative aids. Earlier in 
this thesis (Chapter 6), I identified a number of caveats for doing so based on the results of this doctoral research and the broader lie detection literature. Practitioners must be made aware of the limitations of verbal credibility assessment tools. Research in this thesis also examined the efficacy of a within-statement lie detection technique; namely, verbal baselining. Our results suggest that this may not be an effective method of lie detection. Therefore, at present, police practitioners are not advised to use employ similar techniques in the field.

Altogether, the insights gained from this line of doctoral research could be used to improve tools and techniques for assessing the verbal credibility of statements; in particular by exploiting liars' strategy of embedding lies into truthful stories.

\section{Innovation of the Research}

The research presented in this thesis is part of a small body of scientific literature that has explicitly examined statements that contain a combination of both truthful and deceptive information. This thesis contributed evidence to solidify the idea that liars will strategically incorporate as much truthful information as possible into their accounts. How this strategy may affect the verbal quality of statements was then investigated across two experiments (Chapters 3 and 4). This revealed insights for the reliability of certain criteria associated with verbal credibility assessment tools.

Previous research has extensively examined verbal credibility assessment tools such as CBCA and RM. The novel contribution of this doctoral research was to advance our understanding of how certain verbal criteria may differ between embedded versus fully fabricated lies, and to what extent this occurs as a function of liars' attempts to maintain consistency. By digging deeper into liars' strategies for formulating deceptive accounts, this research provides insight regarding potential mechanisms behind the production of lies.

In addition, research conducted in this thesis was, to our knowledge, the first to examine whether introducing a baseline comparison that was derived from multiple parts of an 
interviewees' statement could facilitate the detection of lies. The findings from this study offer insight into the difficulty of exploiting embedded lies to detect deception and they serve as an innovative addition to research on within-subjects methods of lie detection.

\section{Implementation and Dissemination}

The findings of this thesis have already been disseminated in several ways. All studies that were part of this doctoral research line (Chapters 2 through 5) have been published in international peer-reviewed journals. To encourage accessibility, these articles were published in open-access journals and were also made publically available on ResearchGate. Additionally, all of these studies have been presented at international conferences that were attended by both academic researchers and legal practitioners.

I have also made efforts to communicate my research findings to a wider lay audience. I have actively promoted my research and findings on social media platforms, such as Twitter and LinkedIn. I also wrote a press release with the University of Portsmouth for one of my

doctoral studies (Chapter 2), which was picked up by over 40 international news outlets and 8 blogs, including The Times and the British Psychological Society (BPS)'s Research Digest. Finally, I was interviewed as part of a Canadian Broadcasting Corporation (CBC) RadioCanada documentary on investigative interviewing and deception detection. I hope to continue to conduct research and to communicate scientific findings to diverse audiences throughout my career. I also intend to continue doing outreach activities and presentations to motivate young students to pursue studies in the areas of legal and forensic psychology. 
Appendices 


\section{List of Appendices}

Appendix 1: Supplementary materials (Chapter 2; Study I)

Appendix 2: Supplementary materials (Chapter 3; Study II)

Appendix 3: Supplementary materials (Chapter 4; Study III)

Appendix 4: Supplementary materials (Chapter 5; Study IV)

Appendix 5: Ethical Approval 
Appendix 1: Supplementary materials (Chapter 2; Study I)

1. Questionnaire Definitions

2. Lie Characteristics and Distribution Skewness

3. Questionnaire Part III: Recalling a Serious Lie

a. Deceivers' Strategies as a Function of Rationale

b. The Influence of Lie Rationale on Participants' Preparation

c. Perceived Success of a Serious Lie

4. Exploratory Testing of Liar Characteristics 


\section{Questionnaire Definitions}

After providing informed consent, and prior to beginning the questionnaire, participants were provided the following set of definitions, which they were asked to read carefully and to keep in mind while making responses throughout the questionnaire: (1) "To lie (i.e., deceive) is to successfully or unsuccessfully make a false statement (to another person), without forewarning, with the intention that the statement will be believed to be true (by the other person); misremembering is not the same as lying; a successful lie means that the intended false-belief has been produced, whereas an unsuccessful lie means that the intended false-belief has not been produced." (2) "Strategies for telling lies (i.e., strategies of deception) refer to the selfregulatory method/s that an individual may use to increase their chances of telling a successful lie."

During the questionnaire, participants were asked to indicate how many lies, of several types, they had told within the past 24 hours. We described each of the options as follows: White lies (Lies of this type occur when someone makes an inconsequential false statement with the purpose of easing social interactions), Exaggerations (Lies of this type occur when someone intentionally makes a statement that reports something as being better or worse than it really is), Omissions/Concealment (Lies of this type occur when someone intentionally withholds information, with the purpose of harming or disadvantaging the receiver for the liar's own benefit), Commission/ Fabrications (Lies of this type occur when false information is intentionally presented as if it was true, with the purpose of harming or disadvantaging the receiver for the liar's own benefit), and Embedded lies (Lies of this type occur when a statement contains one or more lies that are incorporated into an otherwise truthful story, for the liar's own benefit).

As well, during the questionnaire participants were asked to rate on a 10-point Likert scale (1 - not important to 10 - very important) how important they consider verbal and nonverbal 
strategies of deception to be for getting away with a lie. We described verbal strategies as: "Relating to the control of an individual's speech to say things that give a credible impression and avoid raising suspicion of possible deception" and nonverbal strategies of deception as: "Relating to the control of an individual's behaviour to display body language that gives a credible impression and avoids raising suspicion of possible deception”. 


\section{Lie Characteristics and Distribution Skewness}

Participants mostly reported telling white lies, and the distribution was non-normally distributed (skewness $=4.94, S E=0.18$; kurtosis $=31.57, S E=0.35$ ). Three participants mentioned telling over ten white lies in the past 24 hours, and their reports accounted for over $20 \%$ of the reported lies of this type. Forty-nine percent of participants reported having told no white lies in the same time frame. The distributions for the other types of lies (e.g., embedded lies, exaggerations) were similarly skewed in their distributions. Regarding the receivers of deception, participants reported lying most often to family members. Again, the distribution was non-normally distributed, with a skewness of $3.18(S E=0.18)$ and a kurtosis of 14.39 ( $S E=$ 0.35). Most participants $(71.1 \%)$ reported not having told any lies to family members, whereas most of those who did lie to family $(36.1 \%)$, told one or two lies. The highest reported number was eight lies told to family, which was only reported by one participant. The distributions for the other receivers of deception (e.g., friends, colleagues) were also skewed. Finally, participants reported telling most lies via face-to-face interactions (skewness $=3.17, S E=0.18$; kurtosis $=$ $13.21, S E=0.35)$. The majority $(61.3 \%)$ did not report any lies during such interactions, whereas most participants who did report lies during face-to-face interactions $(54.7 \%)$ reported only one or two lies. The distributions for the other mediums of deception (e.g., via text message or social media) were also non-normally distributed. 


\section{Questionnaire Part III: Recalling a Serious Lie}

Participants completed an additional section of the questionnaire that asked about a specific, personal instance in which they told a lie. Participants were asked to think back to a time when they told a serious lie and to answer the following questions accordingly. If they could not recall telling a serious lie, participants were given the option to answer the questions hypothetically. Before answering the questions, participants indicated whether they were responding based on an actual memory or regarding a hypothetical scenario. They were also asked to provide an openended description of the reason why they had lied. Afterwards, participants were asked to rate several items on 5-point Likert scales (1 - strongly agree to 5 - strongly disagree): (i) Before telling the lie, I prepared what I would say, (ii) I used one or more strategies when telling the lie, (iii) I was motivated to get away with the lie, and (iv) I was successful in getting away with the lie. Participants also responded to several open-ended questions: (i) Please describe why you did or did not prepare what you would say during your lie, (ii) If you did prepare before telling your lie, how did you prepare? (iii) Please explain the strategy or strategies you used to be convincing with this lie, and (iv) Please describe why you think you were or were not successful in getting away with your lie.

Reliability. The main coder and a second coder coded a randomly selected $20 \%$ of the participants' open-ended responses in order to establish inter-rater reliability regarding the classification of responses into the appropriate categories. This was evaluated using the two-way mixed effects model measuring consistency, which indicated that raters were consistent across all question categories. Specifically, the Single Measures ICCs for question one ("What was the reason that you lied?") ranged from .80 to 1.00 ; for question two ("Please describe why you did or did not prepare what you would say during your lie?") ranged from .53 to .85 ; for question three ("If you did prepare, how did you prepare?") ranged from .65 to .88; for question four ("What strategy or strategies did you use to be convincing with this lie?") ranged from .55 to 
1.00; and for question five ("Please describe why you think you were or were not successful in getting away with your lie?") ranged from .53 to .85 . After confirming that the raters were consistent, the main coder (Principal Investigator) completed the remaining sample of participant responses and only these scores were used in the analysis. 
a. Deceivers' Strategies as a Function of Rationale

We were interested in exploring how people strategically deceive, as a function of their rationale for deceiving. In the final section of the questionnaire, participants were asked to recall a time in which they had told a serious lie. Based on their responses to this question, we created five categories of rationales for deceiving, as follows: (i) for personal gain ( $n=41)$, (ii) to avoid consequence $(n=47)$, (iii) to avoid the situation $(n=34)$, (iv) to benefit another person $(n=22)$, and $(\mathrm{v})$ other/miscellaneous $(n=11)$. We included only those participants who reported to be basing their responses on a real memory, rather than a hypothetical scenario. This led to the exclusion of 39 participants, and thus, our analyses for the current section were conducted on 155 participants.

Overall, participants reported having been motivated to get away with their lie $(M=1.68$, $S D=0.95$ ), and there were no statistically significant differences between the five rationale groups, $F(4,150)=.38, p=.824, \eta \mathrm{P}^{2}=.010 ; B F_{01}=24.39$. Participants also reported to have prepared what they would say prior to telling the lie $(M=1.98, S D=1.14)$; however, there were no differences between the five rationale groups, $F(4,150)=.07, p=.991, \eta \mathrm{P}^{2}=002 ; B F_{01}=$ 36.31

Strategies. Participants reported using one or more strategies when telling their lie $(M=$ $2.12, S D=1.06$ ); however, there were no differences between the five rationale groups in endorsing at least one strategy, $F(4,190)=1.24, p=.296, \eta_{\mathrm{P}}^{2}=.032 ; B F_{01}=6.90$. Supplementary Table 1 provides an overview of the strategy categories that emerged from the qualitative coding of participants' responses to the question regarding the strategies they employed when delivering their lie. Overall, participants across groups endorsed the various strategies a total of 228 times. Those who deceived to avoid a negative consequence or for personal gain reported the most strategies (a total of 57 and 56 strategies, respectively), whereas those who lied for miscellaneous reasons reported the fewest strategies (11 mentioned strategies; see Supplementary Table 1). The 
most frequently endorsed strategies across groups were "Behavioral manipulation" (22.37\% of all reported strategies; e.g., maintaining eye contact, having a steady voice, appearing calm, behaving in the same manner as normal, etcetera) and "The inclusion of certain types of information" (17.98\% of all reported strategies; e.g., providing unverifiable or verifiable details, including timestamps and specific descriptions, etcetera).

Supplementary Table 1

Qualitative strategies for telling a serious lie as a function of rationale

\begin{tabular}{|c|c|c|c|}
\hline Strategy Categories: & $N$ & $M$ & $S D$ \\
\hline Behavioral manipulation & 51 & 0.33 & 0.47 \\
\hline For personal gain & 16 & 0.39 & 0.49 \\
\hline To avoid consequence & 19 & 0.40 & 0.49 \\
\hline To avoid the situation & 9 & 0.26 & 0.45 \\
\hline To benefit another person & 6 & 0.27 & 0.46 \\
\hline Miscellaneous & 1 & 0.10 & 0.32 \\
\hline Including certain types of information & 41 & 0.26 & 0.44 \\
\hline For personal gain & 8 & 0.20 & 0.40 \\
\hline To avoid consequence & 14 & 0.29 & 0.46 \\
\hline To avoid the situation & 14 & 0.42 & 0.50 \\
\hline To benefit another person & 4 & 0.18 & 0.40 \\
\hline Miscellaneous & 1 & 0.10 & 0.32 \\
\hline General strategies & 28 & 0.18 & 0.39 \\
\hline For personal gain & 8 & 0.20 & 0.40 \\
\hline To avoid consequence & 7 & 0.15 & 0.36 \\
\hline To avoid the situation & 5 & 0.15 & 0.36 \\
\hline To benefit another person & 5 & 0.23 & 0.43 \\
\hline Miscellaneous & 3 & 0.30 & 0.48 \\
\hline Keeping the story short and simple & 27 & 0.17 & 0.38 \\
\hline For personal gain & 6 & 0.15 & 0.36 \\
\hline To avoid consequence & 8 & 0.17 & 0.38 \\
\hline To avoid the situation & 8 & 0.24 & 0.43 \\
\hline To benefit another person & 3 & 0.14 & 0.35 \\
\hline Miscellaneous & 2 & 0.20 & 0.42 \\
\hline Telling a plausible and convincing story & 18 & 0.12 & 0.32 \\
\hline For personal gain & 6 & 0.15 & 0.36 \\
\hline To avoid consequence & 7 & 0.16 & 0.36 \\
\hline To avoid the situation & 2 & 0.06 & 0.24 \\
\hline To benefit another person & 2 & 0.09 & 0.29 \\
\hline
\end{tabular}




\begin{tabular}{|c|c|c|c|}
\hline Miscellaneous & 1 & 0.10 & 0.32 \\
\hline Incorporating truthful details & 21 & 0.14 & 0.34 \\
\hline For personal gain & 10 & 0.24 & 0.43 \\
\hline To avoid consequence & 6 & 0.13 & 0.33 \\
\hline To avoid the situation & 2 & 0.06 & 0.24 \\
\hline To benefit another person & 3 & 0.14 & 0.35 \\
\hline Miscellaneous & 0 & 0.00 & 0.00 \\
\hline Omitting certain types of information & 20 & 0.13 & 0.34 \\
\hline For personal gain & 6 & 0.15 & 0.36 \\
\hline To avoid consequence & 3 & 0.06 & 0.24 \\
\hline To avoid the situation & 5 & 0.15 & 0.36 \\
\hline To benefit another person & 5 & 0.23 & 0.43 \\
\hline Miscellaneous & 1 & 0.10 & 0.32 \\
\hline Emotional manipulation & 12 & 0.08 & 0.27 \\
\hline For personal gain & 2 & 0.05 & 0.22 \\
\hline To avoid consequence & 6 & 0.13 & 0.33 \\
\hline To avoid the situation & 2 & 0.06 & 0.24 \\
\hline To benefit another person & 2 & 0.09 & 0.29 \\
\hline Miscellaneous & 0 & 0.00 & 0.00 \\
\hline Not Applicable & 6 & 0.04 & 0.19 \\
\hline For personal gain & 1 & 0.02 & 0.16 \\
\hline To avoid consequence & 1 & 0.02 & 0.14 \\
\hline To avoid the situation & 2 & 0.06 & 0.24 \\
\hline To benefit another person & 0 & 0.00 & 0.00 \\
\hline Miscellaneous & 2 & 0.20 & 0.42 \\
\hline No Strategy & 4 & 0.03 & 0.16 \\
\hline For personal gain & 1 & 0.02 & 0.16 \\
\hline To avoid consequence & 0 & 0.00 & 0.00 \\
\hline To avoid the situation & 1 & 0.03 & 0.17 \\
\hline To benefit another person & 1 & 0.05 & 0.21 \\
\hline Miscellaneous & 1 & 0.10 & 0.32 \\
\hline
\end{tabular}

Note. The $N$ column represents the number of participants who endorsed each category, both in the total sample and for each rationale group, respectively. The total number of endorsed categories surpasses 155 because each participant could report multiple strategies that may have fallen into more than one category. 
b. The Influence of Lie Rationale on Participants' Preparation

To further evaluate whether participants prepared more or less depending on their rationale for lying, we created two groups to represent "preparers" (scores of one and two) and "nonpreparers" (scores of four and five) based on participants' responses to their preparation on a 5point Likert scale. The majority of participants reported to have prepared $(n=125)$ whereas only 24 participants indicated that they did not prepare. Six participants responded in a neutral manner (i.e., scores of three) and thus their data were removed from the analyses. To examine whether there was an association between whether an individual prepares for their deception or not and the rationale for deceiving, we conducted a series of chi square tests of independence. All expected cell frequencies were greater than five. There was not a statistically significant association between preparation and the rationale for deceiving, $\chi^{2}(4)=0.72, p=.948, V=.070$.

Supplementary Table 2 provides an overview of the data regarding participants' coded responses to the open-ended question: "Please describe why you did or did not prepare what you would say during your lie." These data are reported on only the preparer and non-preparer groups. The majority of justifications fell into the categories relating to preparing for the deception; whereas almost a quarter of the reports $(21.12 \%)$ fell into the two categories relating to a lack of preparation (i.e., "No preparation because caught unexpectedly" and "Miscellaneous reasons for not preparing"). Across groups, the most endorsed reason for preparing was to "Ensure the lie is sound and convincing" (17.39\% of reports). The two rationales that led to the highest endorsement of preparation methods were those who told lies to avoid consequence and for personal gain (mentioned 50 and 43 justifications, respectively).

Supplementary Table 2

Justifications for preparing or not preparing as a function of rationale

\begin{tabular}{lccc}
\hline Justifications: & $N$ & $M$ & $S D$ \\
\hline & 28 & 0.19 & 0.39
\end{tabular}


Prepare to ensure the lie is sound and convincing

For personal gain

To avoid consequence

$\begin{array}{lll}5 & 0.12 & 0.33\end{array}$

To avoid the situation

$\begin{array}{lll}9 & 0.19 & 0.40\end{array}$

To benefit another person

0.32

0.48

Miscellaneous

$0.15 \quad 0.37$

$0.10 \quad 0.32$

Prepare to increase the likelihood of getting away with the lie

For personal gain

0.15

0.36

To avoid consequence

0.07

0.26

To avoid the situation

3

10

0.21

0.41

To benefit another person

Miscellaneous

2

0.23

0.43

0.31

1

0.10

0.32

Miscellaneous reasons for preparing

25

0.17

0.38

For personal gain

0.17

0.38

To avoid consequence

7

To avoid the situation 7

To benefit another person

0.15

0.36

Miscellaneous

5

0.16

0.37

$4 \quad 0.20$

0.41

\section{Miscellaneous}

Preparing to control information disclose

2

0.20

0.40

For personal gain

21

To avoid consequence

To avoid the situation

To benefit another person

Miscellaneous

0.14

0.35

8

6

4

3

3

0.20

0.40

0.13

0.34

0.13

0.34

0.15

0.37

0.00

0.00

No preparation because caught unexpectedly

20

0.13

0.34

For personal gain

To avoid consequence

To avoid the situation

To benefit another person

Miscellaneous

0.10

0.30

4
8

8

0.17

0.38

0

6

2

0.00

0.00

0.30

0.47

0.20

0.42

Miscellaneous reasons for not preparing

14

0.09

0.29

For personal gain

5

0.12

0.33

To avoid consequence

To avoid the situation

To benefit another person

0.04

0.20

2

0.06

0.25

Miscellaneous

0.20

0.41

1

0.10

0.32

Not Applicable

30

11

0.20

0.40

For personal gain

0.27

0.45

To avoid consequence

To avoid the situation

To benefit another person

Miscellaneous

0.17

0.38

0.23

0.43

0.05

0.22

0.30

0.48 
Note. The $N$ column represents the number of participants who endorsed each category, both in the total sample and for each rationale group, respectively. The total number of endorsed categories surpasses 149 because each participant could report multiple strategies that may have fallen into more than one category. We coded for an additional category, "Intentionally no preparation to utilise spontaneity"; however, of the data retained in the analyses, there was no endorsement of this category.

Supplementary Table 3 provides an overview of the methods of preparation that arouse from the qualitative coding of participants' responses. Two techniques were reported most often by participants: "Carefully planning a plausible story" (26.49\% of the total reported techniques fell into this category) and "Rehearsing the story" (22.70\% of the total reported techniques). Participants who reported to be deceiving to avoid consequences or for personal gain endorsed the highest frequency of preparation techniques (55 and 52 mentioned techniques, respectively).

Supplementary Table 3

Preparation techniques as a function of rationale

\begin{tabular}{lccc} 
Preparation Techniques: & $N$ & $M$ & $S D$ \\
\hline Carefully plan a plausible story & 49 & 0.32 & 0.47 \\
For personal gain & 12 & 0.29 & 0.46 \\
To avoid consequence & 15 & 0.31 & 0.47 \\
To avoid the situation & 14 & 0.42 & 0.50 \\
To benefit another person & 6 & 0.27 & 0.46 \\
Miscellaneous & 2 & 0.20 & 0.42 \\
& & & \\
Rehearse the story & 42 & 0.27 & 0.45 \\
For personal gain & 9 & 0.22 & 0.42 \\
To avoid consequence & 12 & 0.25 & 0.44 \\
To avoid the situation & 14 & 0.42 & 0.50 \\
To benefit another person & 7 & 0.32 & 0.48 \\
Miscellaneous & 0 & 0.00 & 0.00 \\
& & & \\
Use imagination & 26 & 0.17 & 0.38 \\
For personal gain & 10 & 0.24 & 0.44 \\
To avoid consequence & 7 & 0.15 & 0.36 \\
To avoid the situation & 3 & 0.09 & 0.29 \\
To benefit another person & 5 & 0.23 & 0.43 \\
Miscellaneous & 1 & 0.10 & 0.32 \\
& & & \\
Not Applicable & 24 & 0.15 & 0.36 \\
For personal gain & 7 & 0.17 & 0.38
\end{tabular}


To avoid consequence

To avoid the situation

To benefit another person

No preparation

19

0.12

0.10

0.33

For personal gain

To avoid consequence

To avoid the situation

To benefit another person

Miscellaneous

Gather certain information to include

For personal gain

To avoid consequence

To avoid the situation

To benefit another person

Miscellaneous

Plan the nonverbal behavior

For personal gain

To avoid consequence

To avoid the situation

To benefit another person

Miscellaneous

Note. The $N$ column represents the number of participants who endorsed each category, both in the total sample and for each rationale group, respectively. The total number of endorsed categories surpasses 155 because each participant could report multiple strategies that may have fallen into more than one category. 


\section{c. Perceived Success of a Serious Lie}

Lastly, participants reported to have been successful in getting away with their lie $(M=1.84$, $S D=1.06)$; however, there were no differences between the five rationale groups, $F(4,150)=$ $.60, p=.663, \eta_{\mathrm{P}}^{2}=.016 ; B F_{01}=15.86$. We also examined participants' qualitative responses to the question "Please describe why you think you were or were not successful in getting away with your lie." Supplementary Table 4 provides an overview of the exact values of participants' reasons for believing their deception was successful versus unsuccessful, as a function of their rationale. The qualitative data mirrored participants' Likert response scores; participants most frequently endorsed the category that indicated they thought to have been successful because the receiver believed the lie (34.94\% of the reports; e.g., the receiver did not question further, the receiver's behavior/response indicated acceptance of the story, the receiver was not suspicious, etcetera).

Supplementary Table 4

\begin{tabular}{lccc} 
Reasons related to successful versus unsuccessful deception as & a function of rationale \\
\hline Reasons relating to perceived success: & $N$ & $M$ & $S D$ \\
\hline & & & \\
Successful: Receiver believed the lie & 58 & 0.37 & 0.49 \\
For personal gain & 17 & 0.42 & 0.50 \\
To avoid consequence & 17 & 0.35 & 0.48 \\
To avoid the situation & 14 & 0.41 & 0.50 \\
To benefit another person & 8 & 0.36 & 0.49 \\
Miscellaneous & 2 & 0.20 & 0.42 \\
& & & \\
Successful: Strategic statement and delivery & 43 & 0.28 & 0.45 \\
For personal gain & 13 & 0.32 & 0.47 \\
To avoid consequence & 15 & 0.31 & 0.47 \\
To avoid the situation & 8 & 0.24 & 0.43 \\
To benefit another person & 5 & 0.23 & 0.43 \\
Miscellaneous & 2 & 0.20 & 0.42 \\
& & & \\
Successful: No repercussions & 20 & 0.13 & 0.34 \\
For personal gain & 4 & 0.10 & 0.30 \\
To avoid consequence & 12 & 0.25 & 0.44 \\
To avoid the situation & 3 & 0.09 & 0.29 \\
To benefit another person & 1 & 0.05 & 0.21
\end{tabular}


Not successful: General

For personal gain

$4 \quad 0.10$

To avoid consequence

$2 \quad 0.04$

0.20

To avoid the situation

$4 \quad 0.12$

0.33

To benefit another person

$4 \quad 0.18$

0.39

Miscellaneous

3

0.30

0.48

Neutral: Unaware of deception success

$$
19
$$

0.12

0.33

For personal gain

3

To avoid consequence

To avoid the situation

8

To benefit another person

0.07

0.26

Miscellaneous

$\begin{array}{ll}3 & 0.09\end{array}$

0.38

$5 \quad 0.23$

0.29

Not Applicable

$0 \quad 0.00$

0.43

For personal gain

$\begin{array}{lll}9 & 0.06 & 0.24\end{array}$

To avoid consequence

0.05

0.22

To avoid the situation

$\begin{array}{ll}2 & 0.05 \\ 1 & 0.02\end{array}$

0.14

To benefit another person

$2 \quad 0.06$

0.24

Miscellaneous

$1 \quad 0.05$

0.21

Note. The $N$ column represents the number of participants who endorsed each category, both in the total sample and for each rationale group, respectively. The total number of endorsed categories surpasses 155 because each participant could report multiple strategies that may have fallen into more than one category. 


\section{Exploratory Testing of Liar Characteristics}

Additionally, we explored the effects of sex and education level on self-reported deception ability. First, we evaluated the association between sex and self-reported deception ability by conducting a chi square test of association. In this analysis we used only males and females (95 and 97, respectively), we excluded two participants who responded 'Prefer not to answer' regarding their sex. We observed a significant association between sex and deception ability, $\chi^{2}(2)=12.31, p=.002, V=.253$. We followed up this significant effect by comparing the column proportions. Our results indicate that, of those who self-reported to be Poor liars, $70 \%(n=35)$ were female compared to $30 \%(n=15)$ male. Additionally, of those who identified as Good liars, $62.7 \%(n=42)$ were male whereas $37.3 \%(n=25)$ were female. Both column proportions were significantly different at the .05 level. The distribution of males and females in the Neutral ability group did not differ significantly.

Next, to evaluate participants' level of education, we classified 'high school', 'some college' and 'associate degree' as Low education $(n=71)$, 'bachelor degree' as Moderate education $(n=88)$, and 'graduate or professional degree' as High education $(n=35)$. To examine whether there was an association between education level and self-reported deception ability, we conducted a chi square test of association. There was no significant association between participants' education level and their self-reported deception ability, $\chi^{2}(4)=9.09, p=$ $.059, V=.153$. Taken together, these findings suggest that men are more likely to self-report as Good liars, whereas women are more likely to self-report as Poor liars. Education level did not affect self-reported deception ability. 
Appendix 2: Supplementary materials (Chapter 3; Study II)

1. Instruction Letters One and Two by Condition

2. Structured Interview Question Format

3. Motivation, Preparation, and Self-Perceived Success Analyses

4. Qualitative Coding of Preparation Techniques

5. Clarity of Statements 


\section{Instruction Letters One and Two by Condition ${ }^{8}$}

\section{Instruction Letter One. The specific instructions participants received depended on their conditions, as follows:}

Dear Participant:

Truth-Truth condition:

Please complete the following two tasks:

Task A: Your assignment is to help develop a promotional flyer for __ located in _. Using the camera provided, please make your way to the _ and take photos that can be included on the flyer to promote the café. Please avoid taking photos of the customers. After doing so, please complete Task B.

Task B: Your assignment is to go to the bus stop _ located at _ to search for Michelle (photo attached), who will be arrivins by bus sometime today. Please use the paper and pen provided to write down the information for any buses that arrive and/or depart during your time. If you see Michelle arrive, please take a photo of her using the camera provided. Wait at the bus stop for a minimum of five minutes, and then return to the research room, regardless of whether you find Michelle or not.

II. Please report back to the experimenter in between twenty to thirty-five minutes. You will then receive your next instructions.

Dear Participant:

\section{Lie-Lie condition:}

For the next twenty to thirty-five minutes, please exit the research room and make yourself busy (e.g., study, get a coffee). Once that time is up, please return to the room and you will then receive your next instructions.

Dear Participant:

\section{Truth-Lie condition:}

I. Please complete the following task:

Your assignment is to help develop a promotional flyer for __ located in _.. Using the camera provided, please make your way to the _ and take photos that can be included on the flyer to promote the café. Please avoid taking photos of the customers.

II. Please report back to the experimenter in between twenty to thirty-five minutes. You will then receive your next instructions.

Dear Participant:

$\underline{\text { Lie-Truth condition: }}$

I. Please complete the following task:

Your assignment is to go to the bus stop _ located at _ to search for Michelle (photo attached), who will be arriving by bus sometime today. Please use the paper and pen provided to write down the information for any buses that arrive and/or depart during your time. If you see Michelle arrive, please take a photo of her using the camera provided. Wait at the bus stop for a minimum of five minutes, and then return to the research room, regardless of whether you find Michelle or not.

II. Please report back to the experimenter in between twenty to thirty-five minutes. You will then receive your next instructions.

\footnotetext{
${ }^{8}$ Information regarding specific locations was removed to allow for a blinded review.
} 
Dear Participant:

Truth-Truth condition:

I. For the next phase of this experiment, you will be interviewed by the researcher about the tasks you have completed. Please report your memories truthfully and with as much detail as possible. Please report and answer questions based on having completed both of the following tasks:

- Task A: Your assignment is to help develop a promotional flyer for __ located in _. Using the camera provided, please make your way to the _ and take photos that can be included on the flyer to promote the café. Please avoid taking photos of the customers. After doing so, please complete Task B.

- Task B: Your assignment is to go to the bus stop __ located at __ to search for Michelle (photo attached), who will be arriving by bus sometime today. Please use the paper and pen provided to write down the information for any buses that arrive and/or depart during your time. If you see Michelle arrive, please take a photo of her using the camera provided. Wait at the bus stop for a minimum of five minutes, and then return to the research room, regardless of if you find Michelle or not.

II. Without using the photos or written document, you must convince the interviewer that you completed both Task A and Task B. If you are highly convincing and the interviewer does not suspect you are fabricating your reports, you will be entered into a raffle for the chance to win a $50 € \mathrm{VVV}$ voucher. If you fail to convince the interviewer, you will be required to stay an additional twenty minutes to provide a written account of your statements.

You have ten minutes to prepare your statements. Please do not write any notes.

Dear Participant:

Lie-Lie condition:

For the next phase of this experiment, you will be interviewed by the researcher. Please report and answer questions with as much detail as possible, as if you had completed both of the following tasks:

Task A: Your assignment is to help develop a promotional flyer for _ located in _. Using the camera provided, please make your way to _ and take photos that can be included on the flyer to promote the café. Please avoid taking photos of the customers.

Task B: Your assignment is to go to the bus stop _ located at _ to search for Michelle (photo attached), who will be arriving by bus sometime today. Please use the paper and pen provided to write down the information for any buses that arrive and/or depart during your time. If you see Michelle arrive, please take a photo of her using the camera provided. Wait at the bus stop for a minimum of five minutes, and then return to the research room, regardless of if you find Michelle or not.

You must convince the interviewer that you completed both Task A and Task B. If you are highly convincing and the interviewer does not suspect you are fabricating your reports, you will be entered into a raffle for the chance to win a $50 €$ VVV voucher. If you fail to convince the interviewer, you will be required to stay an additional twenty minutes to provide a written account of your activities over the past hour.

You have ten minutes to prepare your statement. Please do not write any notes. 
Dear Participant:

Truth-Lie condition:

I. For the next phase of this experiment, you will be interviewed by the researcher about the task you completed. Please report your memories truthfully and with as much detail as possible. In addition to reporting about the task you did complete, you must also report that you completed Task B. Therefore, please report and answer questions as if you had completed both of the following tasks:

- Task A: Your assignment is to help develop a promotional flyer for __ located in __. Using the camera provided, please make your way to _ and take photos that can be included on the flyer to promote the café. Please avoid taking photos of the customers. After doing so, please complete Task B.

- Task B: Your assignment is to go to the bus stop __ located at __ to search for Michelle (photo attached), who will be arriving by bus sometime today. Please use the paper and pen provided to write down the information for any buses that arrive and/or depart during your time. If you see Michelle arrive, please take a photo of her using the camera provided. Wait at the bus stop for a minimum of five minutes, and then return to the research room, regardless of if you find Michelle or not.

II. Without using the photos or written document, you must convince the interviewer that you completed both Task A and Task B. If you are highly convincing and the interviewer does not suspect you are fabricating your reports, you will be entered into a raffle for the chance to win a $50 € \mathrm{VVV}$ voucher. If you fail to convince the interviewer, you will be required to stay an additional twenty minutes to provide a written account of your statements.

You have ten minutes to prepare your statements. Please do not write any notes.

Dear Participant:

Lie-Truth condition:

I. For the next phase of this experiment, you will be interviewed by the researcher about the task you completed. Please report your memories truthfully and with as much detail as possible. In addition to reporting about the task you did complete, you must also report that you completed Task A. Therefore, please report and answer questions as if you had completed both of the following tasks:

- Task A: Your assignment is to help develop a promotional flyer for __ located in __. Using the camera provided, please make your way to _ and take photos that can be included on the flyer to promote the café. Please avoid taking photos of the customers.

- Task B: Your assignment is to go to the bus stop __ located at _ to search for Michelle (photo attached), who will be arriving by bus sometime today. Please use the paper and pen provided to write down the information for any buses that arrive and/or depart during your time. If you see Michelle arrive, please take a photo of her using the camera provided. Wait at the bus stop for a minimum of five minutes, and then return to the research room, regardless of if you find Michelle or not.

II. Without using the photos or written document, you must convince the interviewer that you completed both Task A and Task B. If you are highly convincing and the interviewer does not suspect you are fabricating your reports, you will be entered into a raffle for the chance to win a $50 € \mathrm{VVV}$ voucher. If you fail to convince the interviewer, you will be required to stay an additional twenty minutes to provide a written account of your statements.

You have ten minutes to prepare your statements. Please do not write any notes. 


\section{Structured Interview Question Format}

Structured Interview Format. Participants were interviewed according to the following format:

\begin{tabular}{|c|}
\hline Structured Interview Format: \\
\hline PART ONE: (Task A) \\
\hline $\begin{array}{l}\text { I'll ask you to begin by providing a free recall of what you did for Task A and then I will ask you some } \\
\text { specific questions. }\end{array}$ \\
\hline $\begin{array}{l}\text { 1. In as much detail as possible, please walk me through exactly what you did from the moment you } \\
\text { left this room to the moment you finished Task A. }\end{array}$ \\
\hline 2. What else can you tell me about this task? \\
\hline 3. How long did this task take you? \\
\hline 4. $\quad$ Did you find this task difficult? \\
\hline 5. Did anything unexpected happen or perhaps something that didn't go as planned? \\
\hline 6. Without showing me the camera or notes, please describe what I will see when I look at them. \\
\hline PART TWO: (Task B) \\
\hline $\begin{array}{l}\text { I'll now ask you to provide a free recall of what you did for Task B and then I will ask you some specific } \\
\text { questions. }\end{array}$ \\
\hline $\begin{array}{l}\text { 1. In as much detail as possible, please walk me through exactly what you did from the moment you } \\
\text { finished Task B to the moment you walked back to this room. }\end{array}$ \\
\hline 2. What else can you tell me about this task? \\
\hline 3. How long did this task take you? \\
\hline 4. $\quad$ Did you find this task difficult? \\
\hline 5. Did anything unexpected happen or perhaps something that didn't go as planned? \\
\hline 7. Without showing me the camera or notes, please describe what I will see when I look at them. \\
\hline PART THREE: (Task A and B) \\
\hline $\begin{array}{l}\text { 1. Please think back over the entire assignment, and try to remember if there are any other details that } \\
\text { you may have missed. }\end{array}$ \\
\hline
\end{tabular}


2. Motivation, Preparation, and Self-Perceived Success Analyses

To test participants' motivation, preparation, and self-perceived success we conducted a series of 2 (veracity of the first element: [truth, lie]) X 2 (veracity of the second element: [truth, lie] between-subjects ANOVAs. Participants reported to have been highly motivated $(M=1.16$, $S D=0.48)$, with no significant differences for the veracity of the first or the second element, $F(1$, $140)=0.03, p=.864$ and $F(1,140)=0.27, p=.607$, respectively. Participants overall endorsed that they had enough time to prepare for the interview $(M=1.03, S D=0.28)$, with no significant effects for the veracity of the first or second element, $F(1,140)=0.09, p=.763$ and $F(1,140)=$ $0.09, p=.763$, respectively. Additionally, participants reported to have prepared their statements strategically $(M=2.45, S D=1.08)$. A significant main effect emerged for the veracity of the first element, $F(1,140)=5.20, p=.024$, with participants who told deceptive first elements reporting more strategic preparation $(M=2.26, S D=0.96)$ than participants who told truthful second elements $(M=2.65, S D=1.16)$. The veracity of the second element also significantly affected the strategic preparation, $F(1,140)=11.69, p<.001$, with participants who told deceptive second elements reporting more strategic preparation $(M=2.17, S D=0.96)$ than participants who told truthful second elements $(M=2.75, S D=1.12)$. Regarding self-perceived success, participants were somewhat convinced they would be required to stay longer to provide a written statement $(M=3.33, S D=0.97)$. There was a significant effect for the veracity of the first element, $F(1$, $140)=6.98, p=.009$, meaning that participants who reported deceptive first elements were more likely to believe they would have to stay longer $(M=3.13, S D=0.95)$ relative to participants who reported truthful second elements $(M=3.54, S D=0.11)$. We did not observe a significant effect of the veracity of the second element, $F(1,140)=1.99, p=.161$. There were no significant interaction effects (all $p$ 's $>.105)$. 


\section{Qualitative Coding of Preparation Techniques}

Supplementary Table 5 shows the frequency of occurrence of participants' self-reported open-ended interview preparation techniques as a function of veracity condition. Responses were extracted from the Post-Interview Questionnaire, organised into data-driven themes, and then recoded into the appropriate categories. Across all veracity conditions, $40.38 \%$ of the mentioned interview preparation techniques related to "Using imagination to prepare the statement". Examples of techniques within this category include "imagine yourself doing the tasks and/or walking the routes"; "visualise the locations of the tasks"; and "imagine the sequence of events". Roughly one quarter of the mentioned techniques related to "Strategically preparing the statement and/or responses for the interview" (23.46\%; e.g., "anticipate the interview structure and/or questions and prepare responses accordingly"; "practice the interview by yourself"; "construct the chronological order of events"), and approximately another quarter of techniques fell into the category of "Purposefully manipulating the content (quantity/type of details) of the statement" (22.31\%; e.g., "fabricate specific details to be convincing"; "relate to memory and/or previous experiences"; "create a plausible and/or convincing story"). An additional $13.85 \%$ of mentioned techniques fell into an "Other or miscellaneous" category (e.g., "control the quantity of preparation time"; "use information provided in the instructions"; "improvise and be spontaneous"). As shown in Supplementary Table 5, participants in the Lie-Lie condition reported the most strategies whereas those in the Truth-Truth condition reported the least strategies. In general, the frequencies of reported preparation techniques appear relatively consistent across the different veracity conditions. 
Supplementary Table 5

Frequency and percentage of interview preparation techniques across veracity conditions

$$
\text { Condition }
$$

Preparation Techniques $\quad$ Truth-Truth $\quad$ Lie-Lie $\quad$ Truth-Lie Lie-Truth

\begin{tabular}{|c|c|c|c|c|}
\hline Use imagination to prepare the statement & $\begin{array}{c}17 \\
(\mathbf{2 9 . 8 2})\end{array}$ & $\begin{array}{c}38 \\
(\mathbf{5 0 . 6 7})\end{array}$ & $\begin{array}{c}27 \\
(\mathbf{4 0 . 9 1})\end{array}$ & $\begin{array}{c}23 \\
(\mathbf{3 7 . 1 0})\end{array}$ \\
\hline $\begin{array}{l}\text { Strategically prepare the statement and/or } \\
\text { responses for the interview }\end{array}$ & $\begin{array}{c}16 \\
(28.07)\end{array}$ & $\begin{array}{c}13 \\
(17.33)\end{array}$ & $\begin{array}{c}15 \\
(22.73)\end{array}$ & $\begin{array}{c}17 \\
(27.42)\end{array}$ \\
\hline $\begin{array}{l}\text { Purposefully manipulate the content } \\
\text { (quantity/type of details) of the statement }\end{array}$ & $\begin{array}{c}15 \\
(26.32)\end{array}$ & $\begin{array}{c}12 \\
(17.33)\end{array}$ & $\begin{array}{c}14 \\
(21.21)\end{array}$ & $\begin{array}{c}16 \\
(25.81)\end{array}$ \\
\hline Other/Miscellaneous & $\begin{array}{c}9 \\
(15.79)\end{array}$ & $\begin{array}{c}11 \\
(14.67)\end{array}$ & $\begin{array}{c}10 \\
(15.15)\end{array}$ & $\begin{array}{c}6 \\
(9.68)\end{array}$ \\
\hline
\end{tabular}

$\begin{array}{lllll}\text { Total frequency count per condition } & 57 & 75 & 66 & 62\end{array}$

Note. The frequencies and percentages are reported for $N=142$ as two participant's scores were not recorded from the Qualtrics survey report for this question. The bolded numbers represent the category with the largest percentage per veracity condition. 


\section{Clarity of Statements}

We also examined whether veracity influenced the clarity of participants' accounts. A chi-square test of independence was first conducted between the clarity of element one and veracity condition. All expected cell frequencies were greater than five. There was a statistically significant association between the clarity of element one and veracity condition, $\chi^{2}(3)=8.89, p$ $=.031$. The association was moderately strong, $\varphi_{\mathrm{c}}=0.25, p=.031$. Crosstabulation results are presented in Supplementary Table 6. The proportion of individuals whose first element of the statement was scored as clear was significantly higher for those in the Truth-Truth condition than for those in the Lie-Lie condition. Specifically, for individuals whose statement elements were both deceptive, $55.56 \%$ had their first element scored as unclear, whereas for individuals whose statement elements were both truthful, only $22.22 \%$ had their first element scored as unclear. Individuals whose statements contained a mixture of truthful and deceptive information were not significantly different in the evaluation of the clarity of their first element, regardless of the veracity. Thus, it appears that veracity significantly influenced the perceived clarity of interviewees' first elements of statements only when they were reporting entirely truthfully or entirely deceptively.

Supplementary Table 6

Crosstabulation of clarity score and condition for element one

\begin{tabular}{|c|c|c|c|c|}
\hline \multirow[b]{2}{*}{ Clarity Score } & \multirow[b]{2}{*}{ Truth-Truth } & \multicolumn{2}{|c|}{ Condition } & \multirow[b]{2}{*}{ Lie-Trutl } \\
\hline & & Lie-Lie & Truth-Lie & \\
\hline Unclear (0) & $\begin{array}{c}8 \\
(-2.20)\end{array}$ & $\begin{array}{c}20 \\
\mathbf{( 2 . 6 0 )}\end{array}$ & $\begin{array}{c}14 \\
(0.20)\end{array}$ & $\begin{array}{c}12 \\
(-0.60)\end{array}$ \\
\hline Clear (1) & $\begin{array}{c}28 \\
(\mathbf{2 . 2 0})\end{array}$ & $\begin{array}{c}16 \\
(-2.60)\end{array}$ & $\begin{array}{c}22 \\
(-0.20)\end{array}$ & $\begin{array}{c}24 \\
(0.60)\end{array}$ \\
\hline
\end{tabular}

Note. Adjusted residuals appear in parentheses below observed frequencies. Adjusted residuals in bold are those that exceed $+/-2$. 
Next, we conducted a second chi-square test of independence between the clarity of element two and condition, to examine the influence of veracity on the second element of participants' statements. All expected cell frequencies were greater than five. There was a statistically significant association between the clarity of element two and veracity condition, $\chi^{2}(3)=10.36, p=.016$. The association was moderately strong, $\varphi_{c}=0.27, p=.016$. The crosstabulation is presented in Supplementary Table 7. The proportion of individuals whose second element was scored as clear was significantly higher for those in the Truth-Truth condition than for those in the Lie-Lie condition. Specifically, for individuals whose statement elements were both deceptive, $36.54 \%$ had their second elements scored as unclear, whereas for individuals whose elements were both truthful, only $15.38 \%$ had their second element scored as unclear. Individuals whose statements contained a mixture of truthful and deceptive information were not significantly different in the evaluation of the clarity of their second element. Similarly, veracity significantly influenced the perceived clarity of interviewees' second elements only when they were reporting entirely truthfully or entirely deceptively. Thus, statements were not more or less clear when they were preceded by a lie versus preceded by a truth, unless the statement was entirely truthful or deceptive.

\section{Supplementary Table 7}

Crosstabulation of clarity score and condition for element two

\begin{tabular}{lccccc}
\hline \multirow{2}{*}{ Clarity Score } & & \multicolumn{2}{c}{ Condition } & \\
\cline { 3 - 4 } Unclear (0) & Truth-Truth & Lie-Lie & Truth-Lie & Lie-Truth \\
& & 8 & 19 & 16 & 9 \\
Clear (1) & $\mathbf{( - 2 . 2 0 )}$ & $\mathbf{( 2 . 4 0 )}$ & $(1.20)$ & $(-1.60)$ \\
& & & & \\
& 28 & 17 & 20 & 27 \\
& $\mathbf{( 2 . 2 0 )}$ & $\mathbf{( - 2 . 4 0 )}$ & $(-1.20)$ & $(1.60)$
\end{tabular}

Note. Adjusted residuals appear in parentheses below observed frequencies. Adjusted residuals in bold are those that exceed $+/-2$. 
Appendix 3: Supplementary materials (Chapter 4; Study III)

1. Instruction Letters to Participants

2. Structured Interview Format

3. Coding Description: Verbal Content Analysis

4. Hypotheses and Analyses: Participants' Strategies

5. Analyses: Motivation, Preparation, and Self-Perceived Success 


\section{Truth-Teller Condition:}

Dear Participant,

I. Please imagine that you have been called into a police station as a suspect in a violent burglary that occurred on __ $[D A T E] \_$between approximately $1-3 \mathrm{pm}$. The police have reason to believe that you may have been involved, so they have requested that you provide an alibi statement for your whereabouts on this day.

II. You are innocent of the crime in question; therefore, your task is to convince the interviewer of your innocence by providing a truthful alibi. Read the following instructions carefully:

$>$ Think back to the day that you completed the following activity/activities:

[ACTIVITY]

Please report

exactly what you did on this day (from morning to evening), being sure to include the activity/activities between 1-3pm.

III. During your interview, please provide a truthful statement and answer questions with as much detail as possible, including time stamps wherever possible. If you are highly convincing and the interviewer does not suspect you are lying, you will be entered into a raffle for the chance to win a $50 £$ voucher. If you fail to convince the interviewer, you will be required to stay an additional twenty minutes to provide a written account of your statement.

You have up to ten minutes to prepare your statement. Please do not write any notes.

\footnotetext{
${ }^{9}$ The blank spaces were filled in by the lead researcher based on the selected alibi activity and date.
} 


\section{Fabricated Liar Condition:}

Dear Participant,

I. Please imagine that you have been called into a police station as a suspect in a violent burglary that occurred on ___ $[D A T E] \_$between approximately $1-3 \mathrm{pm}$. The police have reason to believe that you may have been involved, so they have requested that you provide an alibi statement for your whereabouts on this day.

II. You are guilty of the crime in question, and your task is to try and convince the interviewer that you are innocent by providing a completely deceptive alibi. Read the following instructions carefully:

$>$ Please do not report what you actually did on [DATE] ; rather, make up a false statement to explain what you did that day (from morning to evening), including the deceptive story that you completed the following activity/activities from approximately 1-3pm:

\section{[ACTIVITY]}

III. During your interview, please report and answer questions with as much detail as possible, including time stamps wherever possible. If you are highly convincing and the interviewer does not suspect you are lying, you will be entered into a raffle for the chance to win a $50 £$ voucher. If you fail to convince the interviewer, you will be required to stay an additional twenty minutes to provide a written account of your statement.

You have up to ten minutes to prepare your statement. Please do not write any notes. 


\section{Embedded Liar Condition:}

Dear Participant,

I. Please imagine that you have been called into a police station as a suspect in a violent burglary that occurred on___ $[D A T E] \_$_ between approximately $1-3 \mathrm{pm}$. The police have reason to believe that you may have been involved, so they have requested that you provide an alibi statement for your whereabouts on this day.

II. You are guilty of the crime in question, and your task is to try and convince the interviewer that you are innocent by providing a partially deceptive alibi.

Read the following instructions carefully:

$>$ Please truthfully report what you actually did on [DATE] (from morning to evening), and you must also incorporate into this truthful statement the deceptive story that you completed the following activity/activities from approximately 1-3pm: [ACTIVITY]

III. During your interview, please report and answer questions with as much detail as possible, including time stamps wherever possible. If you are highly convincing and the interviewer does not suspect you are lying, you will be entered into a raffle for the chance to win a $50 £$ voucher. If you fail to convince the interviewer, you will be required to stay an additional twenty minutes to provide a written account of your statement.

You have up to ten minutes to prepare your statement. Please do not write any notes. 


\section{Structured Interview Format}

The interviewer began by stating that her goal was to obtain as much information as possible and to determine how credible the participant's alibi was. She instructed the participant to report as many details as possible, even if s/he did not think they were important. She advised the participant to include time stamps wherever possible.

\begin{tabular}{|l|}
\hline \multicolumn{1}{|c|}{ Structured Interview Format: } \\
\hline $\begin{array}{l}\text { In as much detail as possible, please walk me through everything that you did - from } \\
\text { morning to evening - }\end{array}$ \\
\hline What else can you tell me about that day? \\
\hline $\begin{array}{l}\text { Being as detailed as possible, please tell me what you were doing from approximately } 1 \text { to } \\
\text { 3pm. }\end{array}$ \\
\hline $\begin{array}{l}\text { On this day, did anything unexpected happen or perhaps something that didn't go as } \\
\text { planned? }\end{array}$ \\
\hline $\begin{array}{l}\text { Please think back over the entire day, and try to remember if there are any other details that } \\
\text { you may have forgotten or missed. }\end{array}$ \\
\hline
\end{tabular}

\footnotetext{
${ }^{10}$ The specific day that participants were questioned about was determined based on the event they had been
} matched to. 


\section{Coding Description: Verbal Content Analysis}

Coding. Verbal content analysis. The video recorded interviews were transcribed verbatim. The Principal Investigator who has expertise with verbal statement analysis extensively trained a second coder, who was blind to the experimental conditions, to assess statements for several cues. First, statements were assessed for the richness of detail by coding the presence of: (i) spatial information: Information about locations (e.g., "At Wetherspoon pub") or the spatial arrangement of people and/or objects (e.g., "Sitting in the row behind my friend"); (ii) temporal information: Information about when the activities or event happened (e.g., "It was 6:00pm"), duration of an activity (e.g., "We were in the restaurant for one hour"), or an explicit description of a sequence of events (e.g., "I started to do revision notes after I finished eating dinner"); and (iii) perceptual information: Information about what the interviewee saw (e.g., "I saw him sitting at the bar"), smelled (e.g., "It smelled like my roommate had made coffee"), heard (e.g., "He ordered a glass of wine"), tasted (e.g., "The gelato was sweet), or felt (e.g., "The sunshine felt really warm") during their statement. The coding scheme was derived from the RM literature (Johnson \& Raye, 1981) and similar schemes have been used in number of deception studies (e.g., Nahari, 2018a). The richness of detail score represents the sum total of the frequency of occurrences of spatial, temporal, and/or perceptual details.

Next, we coded for the verifiability of detail by examining statements for activities that met the following criteria: (i) they were documented and therefore potentially checkable (e.g., receipt of purchase), (ii) they were carried out together with (an) other identified person(s), rather than alone or with a stranger who could not easily be identified (e.g., study group meeting with specifically named individuals), (iii) they pertained to something that was witnessed by (an) other identified person(s) (e.g., having your professor arrive late to an exam), (iv) they were reported as being recorded by the interviewee (e.g., leaving a voicemail), (v) they used technology (e.g., use of a phone or computer), or (vi) they could potentially be checked by blood analysis and 
medical tests (e.g., drinking alcohol). The verifiability of detail score represents the sum total of the frequency of occurrence of all details that met one or more criteria to be considered verifiable. These criteria were drawn from the VA literature (Boskovic, Bogaard, Merckelbach, Vrij \& Hope, 2017; Nahari et al., 2014a, 2014b). We did not include the information protocol, which is part of the complete VA procedure, because it was outside of the scope of the current study.

The overall statement quality was also assessed by evaluating (i) the clarity and vividness of the statement on a 3-point Likert scale, where 0 is not at all clear and vivid; 1 is somewhat clear and vivid, and 2 is very clear and vivid. Evaluators were given the following prompt: "Do you have a clear, vivid idea of what the interviewee did that day or is it vague and dim?" The second component of statement quality was evaluated by (ii) the plausibility of the statement, again measured on a 3-point Likert scale (i.e., 0 - not at all plausible, 1 - somewhat plausible, and 2 - very plausible). Evaluators were told to consider the following: "Does the interviewees' statement make plausible, realistic sense and follow a logical structure or is it unrealistic and non-logical?" The statement quality variable represents the combined total of both the clarity and plausibility scores. Both of these cues relate to the RM literature and have been examined in a number of other studies (e.g., DePaulo et al., 2003; Johnson \& Raye, 1981; Leal et al., 2015; Sporer \& Küpper, 1995; Zhou et al., 2004).

Statements were assessed for the presence of complications, or rather, occurrences (caused by the interviewee, a third person, or another situational factor) that make a situation more difficult than necessary, often characterised by disrupted activity, failing efforts, failures to progress and activity, suddenly appearing people and/or obstacles, etcetera (e.g., missing the bus or making an unplanned detour during a trip). This cue was originally based on CBCA literature (Steller \& Köhnken, 1989), but the more comprehensive definition was derived from Vrij, Leal, Jupe, \& Harvey (2018). Evaluators also coded for common knowledge details, which are statements that strongly invoke common stereotypical knowledge about everyday events with an 
absence of personalised, experienced, or unique information (e.g., "We went to pick up food at the store"), and self-handicapping strategies, relating to the presence of explicit or implicit justifications made by the interviewee for why they cannot provide certain information (e.g., "My friend chose the restaurant, so I can't remember the name”). In line with previous research (e.g., Vrij, Leal, Jupe et al., 2018), we calculated the tion of complications by computing a total score (number of complications + number of common knowledge details + number of selfhandicapping strategies) and dividing the number of complications by this total score. Scores above .50 specify that the participants reported more complications than the sum total of common knowledge details and self-handicapping strategies, while scores below .50 indicate that the participants reported more common knowledge details and self-handicapping strategies (when summed) than complications. The coding scheme for these criteria was modelled after the work of Vrij, Leal, Mann, et al., 2017 in particular (cf. Sporer, 2016; Volbert \& Steller, 2014; Vrij, Leal, Jupe et al., 2018). 


\section{Hypotheses and Analyses: Participants' Strategies}

Hypotheses. We hypothesised that participants would self-report using strategies to enhance their credibility. In line with previous research, we expected participants who provided truthful statements to report strategies relating to forthcomingness, and providing clear and plausible accounts (Hypothesis 3a); whereas participants who provided entirely fabricated statements would report strategies relating to avoidance, providing plausible accounts, and reporting from previous experience (Hypothesis 3b). Lastly, we predicted that liars who embedded their deception would report an attempt to keep the lie as close to the truth as possible and to match the type and amount of details in the fabricated element of interest to those provided in the truthful elements of their statement (Hypothesis 3c); whereas liars who fabricated their statements entirely would report strategies relating to avoidance and telling a simple, plausible, and convincing story specifically for the critical statement of interest (Hypothesis 3d).

\section{Method}

In the main section of the questionnaire, participants responded to three open-ended questions regarding their preparation and their strategies during the interview (i.e., preparation techniques overall, strategies for the general statement and for the critical statement of interest from 1:00pm to 3:00pm). Lastly, they answered a multiple-response question regarding their use of verbal strategies throughout the interview.

To code the participants' self-reported responses, the Principal Investigator examined the open-ended responses for all questions to establish data-driven categories (see the Results section for a detailed description of the categories). First, we identified each participant's strategy or strategies, then all overlapping responses were combined, and we condensed these strategies into several dominant categories that shared theoretical similarities. The main coder completed each stage of this process and all authors approved upon the final categories. For each of the three 
questions, between seven and eight categories emerged from this coding method (see Supplementary Tables 8 through 10).

The main coder and a second coder then coded a randomly selected $20 \%$ of the participants' open-ended responses to establish inter-rater reliability regarding the classification of responses into the appropriate categories. This was evaluated using the two-way mixed effects model measuring consistency (e.g., Koo \& Li, 2016), which indicated that raters were moderately to highly consistent across all question categories. Specifically, the Single Measures ICCs for question one ("For your interview overall, did you use any strategies to convince the interviewer that you were innocent?") ranged from .59 to 1.00; for question two ("For the critical alibi component [i.e., the period from 1:00pm to $3: 00 \mathrm{pm}$ ], did you use any strategies to convince the interviewer that you were innocent?") ranged from .42 to 1.00 ; for question three ("How did you prepare for the interview?") ranged from .50 to 1.00 . After confirming that the raters were consistent, the second coder completed the remaining sample of participant responses and only these scores were used in the analysis.

\section{Results}

\section{Interviewees' Strategies}

Overall interview strategies. We expected participants to report using strategies to enhance their credibility. Supplementary Figure 1 provides an overview of the endorsement of various strategies for the entire statement across conditions. The overwhelming majority of participants endorsed using strategies. In fact, nearly the entire sample endorsed using at least one strategy for delivering their statement; less than $1 \%$ of the endorsements related to not using a strategy. We first examined differences in strategy use between only the Truth-Tellers and Complete fabrication conditions. With regards to the self-reported endorsement of several strategies, we observed support for our prediction that truthful participants' most frequently reported strategies would be (i) forthcomingness and (ii) providing a clear and simple account 
(Hypothesis 3a). We also found partial support for Hypothesis 3b. The most frequently endorsed strategies by these liars were (i) to report a plausible story, (ii) to keep their statement clear and simple, and (iii) to report from previous memory and experience. The avoidance strategy, however, was endorsed only $4 \%$ of the time. Next, we examined the strategy use of participants in the Embedded lie condition. We found some evidence to support our prediction that participants in this condition would report an attempt to match the type and amount of details in the critical deceptive elements of interest to those provided in the truthful elements of their statement (Hypothesis 3c). The fourth most popular strategy endorsed by participants in this condition was to "Match the deceptive details to the truthful details, in terms of the type of information" (10.46\% of all endorsed strategies). The strategy of "Matching the deceptive details to the truthful details, in terms of the amount of information" was endorsed less, constituting only approximately $7 \%$ of the endorsed strategies by embedded liars. Taken together, these data provide partial support for Hypotheses 3a-c. We replicated patterns observed in previous research regarding the general strategies of truth-telling and lying interviewees, with the exception being that liars in our study did not frequently endorse the avoidance strategy. We also observed partial support for our exploratory prediction regarding the matching of deceptive details to truthful details by participants who embedded their lie into an otherwise truthful account. 


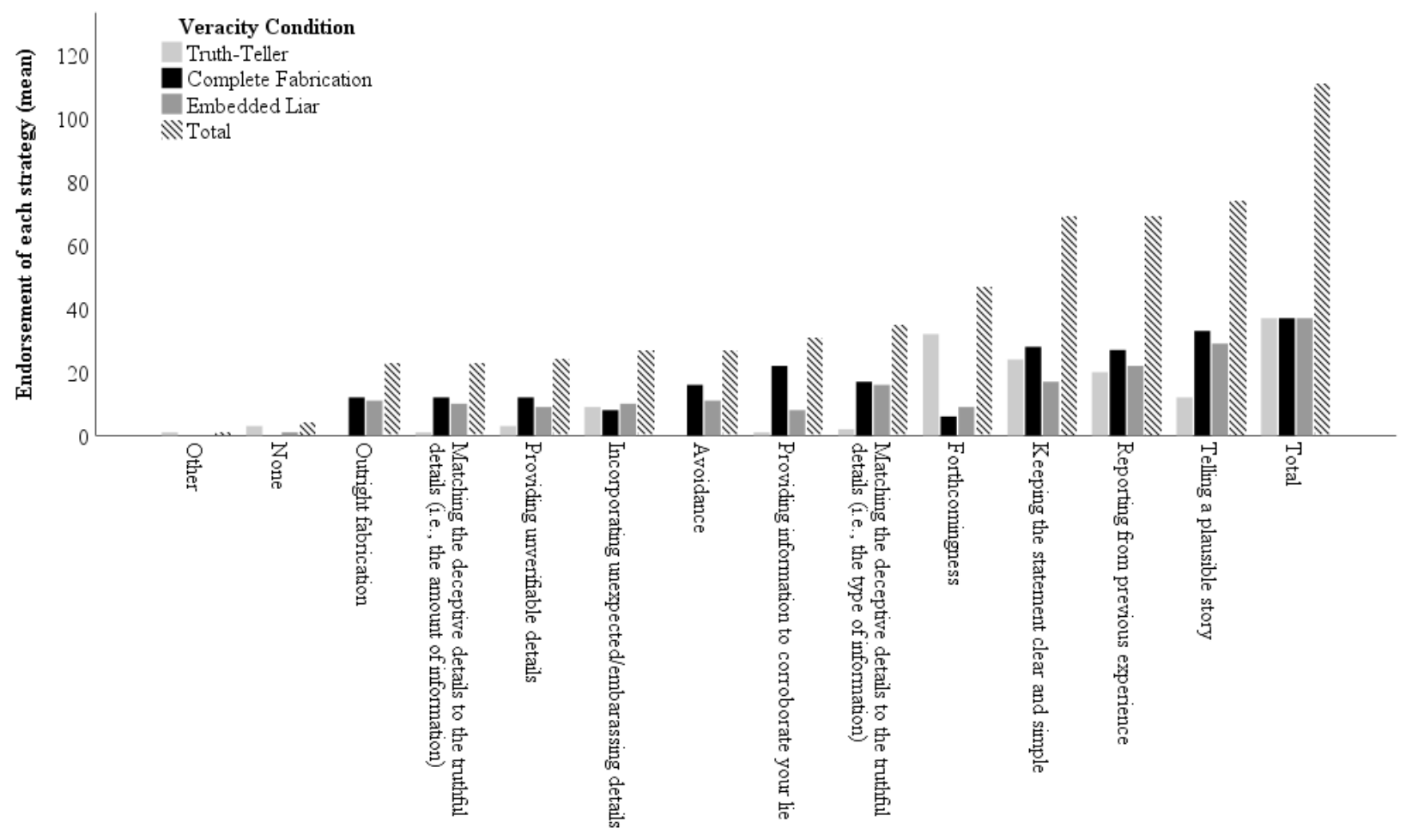

Supplementary Figure 1. Participants' endorsement of verbal strategies as a function of condition

Besides the strategy endorsement from the multiple-response question reported in the previous paragraph, we also examined participants' open-ended responses regarding their interview strategies for the overall interview. Supplementary Table 8 provides an overview of the coded qualitative data. Across all veracity conditions, the two most frequently mentioned interview strategies related to "Providing certain types of specific details" (24.87\% of all reported strategies), and to "Reporting a plausible, detailed, and chronological story" (21.16\%). Only $11.64 \%$ of the reports fell into the category of "Purposefully including truthful details" (e.g., providing many truthful details, including details from previous experiences, etcetera). To examine this further, we conducted a univariate ANOVA with veracity condition as the independent variable and the strategic inclusion of truthful details category as the dependent variable. Participants endorsed this strategy infrequently $(M=0.20, S D=0.48)$, and there were 
no significant differences between the veracity conditions, $F(2,108)=3.05, p=.052, \eta_{\mathrm{P}}^{2}=.053$, $\left.B F_{01}=1.00\right)$. Thus, it appears that participants across conditions reported similarly in their attempts to stay close to the truth within their general alibi statements.

Supplementary Table 8

Frequency and percentage of general interview strategies across veracity conditions

\begin{tabular}{|c|c|c|c|}
\hline Interview Strategy & Truth-Teller & $\begin{array}{c}\text { Condition } \\
\text { Embedded Lie }\end{array}$ & Complete Fabrication \\
\hline $\begin{array}{l}\text { Provide certain types of specific } \\
\text { detail }\end{array}$ & $\begin{array}{c}14 \\
(24.56)\end{array}$ & $\begin{array}{c}17 \\
(28.81)\end{array}$ & $\begin{array}{c}16 \\
(23.19)\end{array}$ \\
\hline $\begin{array}{l}\text { Report a detailed, plausible, } \\
\text { chronological story }\end{array}$ & $\begin{array}{c}17 \\
(29.82)\end{array}$ & $\begin{array}{c}9 \\
(15.25)\end{array}$ & $\begin{array}{c}14 \\
(20.29)\end{array}$ \\
\hline Behavioural control & $\begin{array}{c}11 \\
(19.30)\end{array}$ & $\begin{array}{c}12 \\
(20.34)\end{array}$ & $\begin{array}{c}10 \\
(14.49)\end{array}$ \\
\hline $\begin{array}{l}\text { Purposefully include some } \\
\text { truthful details }\end{array}$ & $\begin{array}{c}2 \\
(3.51)\end{array}$ & $\begin{array}{c}8 \\
(13.56)\end{array}$ & $\begin{array}{c}12 \\
(17.39)\end{array}$ \\
\hline Miscellaneous & $\begin{array}{c}3 \\
(5.26)\end{array}$ & $\begin{array}{c}5 \\
(8.47)\end{array}$ & $\begin{array}{c}7 \\
(10.14)\end{array}$ \\
\hline No strategy & $\begin{array}{c}2 \\
(3.51)\end{array}$ & $\begin{array}{c}3 \\
(5.08)\end{array}$ & $\begin{array}{c}5 \\
(7.25)\end{array}$ \\
\hline $\begin{array}{l}\text { Omit certain types of } \\
\text { information/Vagueness }\end{array}$ & $\begin{array}{c}1 \\
(1.75)\end{array}$ & $\begin{array}{c}3 \\
(5.08)\end{array}$ & $\begin{array}{c}5 \\
(7.25)\end{array}$ \\
\hline Tell the entire truth & $\begin{array}{c}7 \\
(12.28)\end{array}$ & $\begin{array}{c}2 \\
(3.39)\end{array}$ & $\begin{array}{c}0 \\
(0)\end{array}$ \\
\hline $\begin{array}{l}\text { Total frequency count per } \\
\text { condition }\end{array}$ & 57 & 59 & 69 \\
\hline
\end{tabular}

Note. The numbers reported represent the frequency occurrence of each strategy. The respective percentage within each condition is presented in brackets. Frequencies may add up to over 37 (the number of participants in each condition) because each participant could report multiple strategies that may have fallen into one or more categories. The bolded numbers represent the category with the largest percentage per veracity condition.

Interview strategies for the critical alibi component. We were also interested in interviewees' strategies particularly for the critical component of their alibi statement. 
Supplementary Table 9 provides an overview of the participants' open-ended responses regarding their interview strategies for the $1: 00 \mathrm{pm}$ to $3: 00 \mathrm{pm}$ period of their statement. Across veracity conditions, the most frequently mentioned interview strategies related to "Including specific and/or verifiable information" (34.42\% of all reported strategies), and to "Providing a simple, plausible, and convincing story" (19.48\%). Of the reports, $13.64 \%$ fell into the category of "Staying as close to the truth as possible" (e.g., providing many truthful details, including details from previous experiences, etcetera). This contributes further support to Hypothesis 3c, that liars who embedded their deception into an otherwise truthful account would report an attempt, for the critical component of their statement, to stay close to the truth. Indeed, the most frequently endorsed strategy by embedded liars, nearly $26 \%$ of all endorsed strategies within this condition, was to stay as close to the truth as possible. We conducted a univariate ANOVA with veracity condition as the independent variable and the inclusion of truthful details strategy as the dependent variable. Of all the categories, participants' responses infrequently related to this strategy $(M=0.19, S D=0.48)$; however, there were significant differences between the veracity conditions, $\left.F(2,108)=4.69, p=.011, \eta_{\mathrm{P}}^{2}=.080, B F_{10}=3.74\right)$. Post hoc comparisons indicated that the mean difference in reporting this strategy was statistically significant between the Embedded lie condition and the Truth-Teller condition $(0.27,95 \%$ CI [0.01, 0.53], $p=.040)$, and between the Embedded lie condition and the Complete fabrication condition $(0.30,95 \%$ CI [0.04, $0.56], p=.020)$. Thus, participants who embedded their lie reported to have strategically stayed close to the truth significantly more often than participants who provided either entirely truthful or entirely fabricated accounts. Regarding Hypothesis 3d, we expected liars who fabricated their entire story to, for the critical element of interest, report strategies relating to avoidance and telling a simple, plausible, and convincing story. Related to avoidance, the strategy category of "Omitting certain information/Vagueness" was very infrequently endorsed by participants; in fact, it was mentioned by only one participant in this condition. Our data did indicate that 
complete fabricators, more so than any other group, reported to have strategically "Provided a simple, plausible, convincing story" (22\% of the endorsed strategies within this condition). Thus, we received partial support for Hypothesis $3 \mathrm{~d}$.

\section{Supplementary Table 9}

Frequency and percentage of interview strategies for the critical 1:00pm to 3:00pm component of the alibi across veracity conditions

\begin{tabular}{|c|c|c|c|}
\hline \multirow[b]{2}{*}{ Interview Strategy } & \multirow[b]{2}{*}{ Truth-Teller } & \multicolumn{2}{|c|}{ Condition } \\
\hline & & Embedded Lie & Complete Fabrication \\
\hline $\begin{array}{l}\text { Include specific or verifiable } \\
\text { information }\end{array}$ & $\begin{array}{c}21 \\
(42.0)\end{array}$ & $\begin{array}{c}9 \\
(16.67)\end{array}$ & $\begin{array}{c}23 \\
(46.0)\end{array}$ \\
\hline $\begin{array}{l}\text { Provide a simple, plausible, } \\
\text { convincing story }\end{array}$ & $\begin{array}{c}9 \\
(18.0)\end{array}$ & $\begin{array}{c}10 \\
(18.52)\end{array}$ & $\begin{array}{c}11 \\
(22.0)\end{array}$ \\
\hline Stay as close to the truth as possible & $\begin{array}{c}4 \\
(8.0)\end{array}$ & $\begin{array}{c}14 \\
(25.93)\end{array}$ & $\begin{array}{c}3 \\
(6.0)\end{array}$ \\
\hline No strategy & $\begin{array}{c}8 \\
(16.0)\end{array}$ & $\begin{array}{c}4 \\
(7.41)\end{array}$ & $\begin{array}{c}3 \\
(6.0)\end{array}$ \\
\hline Miscellaneous & $\begin{array}{c}3 \\
(6.0)\end{array}$ & $\begin{array}{c}4 \\
(7.41)\end{array}$ & $\begin{array}{c}5 \\
(10.0)\end{array}$ \\
\hline Use consistency and repetitions & $\begin{array}{c}2 \\
(4.0)\end{array}$ & $\begin{array}{c}6 \\
(11.11)\end{array}$ & $\begin{array}{c}2 \\
(4.0)\end{array}$ \\
\hline Behavioural cues & $\begin{array}{c}2 \\
(4.0)\end{array}$ & $\begin{array}{c}3 \\
(5.56)\end{array}$ & $\begin{array}{c}2 \\
(4.0)\end{array}$ \\
\hline $\begin{array}{l}\text { Omit certain } \\
\text { information/Vagueness }\end{array}$ & $\begin{array}{c}1 \\
(2.0)\end{array}$ & $\begin{array}{c}4 \\
(7.41)\end{array}$ & $\begin{array}{c}1 \\
(2.0)\end{array}$ \\
\hline $\begin{array}{l}\text { Total frequency count per } \\
\text { condition }\end{array}$ & 50 & 54 & 50 \\
\hline
\end{tabular}

Note. The numbers reported represent the frequency occurrence of each strategy. The respective percentage within each condition is presented in brackets. Frequencies may add up to over 37 (the number of participants in each condition) because each participant could report multiple strategies that may have fallen into one or more categories. The bolded numbers represent the category with the largest percentage per veracity condition. 


\section{Interview Preparation}

Lastly, we examined interviewees' reported interview preparation techniques (Supplementary Table 10). For all participants, the most frequently mentioned preparation techniques related to "Recalling the events of the day" (30\% of all reported strategies), and to "Planning a plausible story" (25.53\%). Only $8.24 \%$ of strategies mentioned related to the category of "Drawing from previous experience or memory".

Supplementary Table 10

Frequency and percentage of interview preparation techniques across veracity conditions Condition

\begin{tabular}{|c|c|c|c|}
\hline \multirow[b]{2}{*}{ Interview Strategy } & \multicolumn{3}{|c|}{ Condition } \\
\hline & Truth-Teller & Embedded Lie & Complete Fabrication \\
\hline Recall the events of the day & $\begin{array}{c}29 \\
(54.72)\end{array}$ & $\begin{array}{c}14 \\
(23.73)\end{array}$ & $\begin{array}{c}8 \\
(13.79)\end{array}$ \\
\hline Plan a plausible story & $\begin{array}{c}0 \\
(0)\end{array}$ & $\begin{array}{c}18 \\
(30.51)\end{array}$ & $\begin{array}{c}22 \\
(37.93)\end{array}$ \\
\hline $\begin{array}{l}\text { Remember as many specific details } \\
\text { as possible }\end{array}$ & $\begin{array}{c}16 \\
(30.19)\end{array}$ & $\begin{array}{c}6 \\
(10.17)\end{array}$ & $\begin{array}{c}5 \\
(8.62)\end{array}$ \\
\hline Rehearse and/or imagine the story & $\begin{array}{c}4 \\
(7.55)\end{array}$ & $\begin{array}{c}13 \\
(22.03)\end{array}$ & $\begin{array}{c}8 \\
(13.79)\end{array}$ \\
\hline $\begin{array}{l}\text { Draw from previous experience } \\
\text { and memory }\end{array}$ & $\begin{array}{c}0 \\
(0)\end{array}$ & $\begin{array}{c}3 \\
(5.08)\end{array}$ & $\begin{array}{c}11 \\
(18.97)\end{array}$ \\
\hline Miscellaneous & $\begin{array}{c}0 \\
(0)\end{array}$ & $\begin{array}{c}3 \\
(5.08)\end{array}$ & $\begin{array}{c}4 \\
(6.9)\end{array}$ \\
\hline No preparation techniques & $\begin{array}{c}4 \\
(7.55)\end{array}$ & $\begin{array}{c}2 \\
(3.39)\end{array}$ & $\begin{array}{c}0 \\
(0)\end{array}$ \\
\hline $\begin{array}{l}\text { Total frequency count per } \\
\text { condition }\end{array}$ & 53 & 59 & 58 \\
\hline
\end{tabular}

Note. The numbers reported represent the frequency occurrence of each strategy. The respective percentage within each condition is presented in brackets. Frequencies may add up to over 37 (the number of participants in each condition) because each participant could report multiple strategies that may have fallen into one or more categories. The bolded numbers represent the category with the largest percentage per veracity condition. 


\section{Discussion}

\section{Interviewees' Strategies}

Participants in our study overwhelmingly reported to have used strategies to enhance their credibility. In line with previous literature which suggests that innocent interviewees rely on their memory to reconstruct their statement and to provide complete and honest accounts (Hartwig, Granhag, \& Strömwall, 2007), truthful interviewees in our study reported to have been forthcoming and to have provided clear and simple accounts. Our data also indicated that liars who fabricated their entire accounts reported to provide plausible, clear, and simple stories, both for their overall statement as well as for the critical element, a finding that is consistent with past research (e.g., Granhag, Strömwall, \& Jonsson, 2003; Vrij, Granhag, \& Mann, 2010). For their statement overall, liars in our study did not report to use strategies relating to avoidance, which contradicts previous research on deceivers' strategies (e.g., Alison, Alison, Noone, Elntib, Waring, \& Christiansen, 2014; Granhag, Clemens, \& Strömwall, 2009). Interestingly, liars did report the avoidance, or intentional vagueness, strategy specifically for the critical component of their alibi. This is fitting, given that liars in our study knew that the 1:00pm to 3:00pm period was critical, and thus, they may been particularly careful about regulating their disclosure during that period. Participants' attempts to include checkable information provides insight into their metacognitions and contributes anecdotal support to the VA.

Further, we observed that for interviewees' who embedded their lie into a truthful statement, the most reported strategy was to stay as close to the truth as possible. This fits our hypothesis and the findings of previous research; for example, Strömwall and Willén (2011) examined the reported deception strategies by criminal offenders, and found that the most frequently reported strategy was to stay close to the truth. Additionally, we found partial support for our hypothesis that embedded liars attempted to match the type and amount of details in the fabricated elements to those provided in the truthful elements of their statement. 
Taken together, these data provide further insight into how interviewees formulate their accounts, knowledge which contributes to the development of strategy-based methods of lie detection. Moreover, it provides additional evidence to suggest that liars purposefully include truthful information in their statements, even when instructed to provide an entirely fabricated account, a finding that likely extends to deception research more generally. 
5. Analyses: Motivation, Preparation, and Self-Perceived Success

Participants reported to have been highly motivated $(M=4.65, S D=0.72), F(2,108)=0.15$, $\left.p=.858, \eta_{\mathrm{P}}^{2}=.003\right)$. Participants noted that the instructions were clear $(M=4.86, S D=0.48)$, that they had enough time to prepare for the interview $(M=4.90, S D=0.45)$, and that they had prepared their statements somewhat strategically $(M=3.85, S D=1.08)$. There were no significant differences between veracity conditions, $F(2,108)=0.83, p=.438, \eta_{\mathrm{P}}^{2}=.015, F(2$, $108)=1.27, p=.284, \eta_{\mathrm{P}}^{2}=.023$, and $F(2,108)=2.31, p=.104, \eta_{\mathrm{P}}^{2}=.041$, respectively. Participants also indicated that the interviewer was friendly $(M=4.88, S D=0.42), F(2,108)=$ $0.201, p=.819, \eta_{\mathrm{P}}^{2}=.004$. 
Appendix 4: Supplementary materials (Chapter 5; Study IV)

1. Experimental Instructions

2. Cues to Veracity 


\section{Experimental Instructions}

Please imagine yourself in the role of a district detective in the Criminal Investigation Department of the Limburg Police. You are the lead detective for an investigation into a violent burglary that occurred approximately one week ago. Your colleague just finished interviewing a suspect, and your job is to review the interview statement and to assess the suspect's credibility. The interviewee has provided an alibi statement for the entire day in question, from morning to evening, but the critical period of time (i.e., the "statement of interest") that you are most interested in is from 1:00pm to 3:00pm.

Your task is to read the statement carefully and to make a decision about the truthfulness of the critical statement of interest (i.e., the highlighted information, the period of time from 1:00pm to 3:00pm). You will then respond to several questions regarding your decision.

It is extremely important that your decision is correct, if not, either the perpetrator gets away with the crime OR you may send an innocent person to jail. Plus, if you make the correct decisions regarding truthfulness, you will be entered into a raffle to win $€ 50$...

$\underline{\text { Additional instruction for Baseline-present condition: }}$

As the lead investigator, you have access to other sources of information for this case. This evidence confirms that the "general" alibi statement before 1:00pm and after 3:00pm is $\underline{\text { truthful. }}$ Please use this knowledge to compare the "general" part of the interviewees' alibi to the "statement of interest from 1:00pm to $3: 00 \mathrm{pm})$." Try to identify any patterns or changes in the verbal content between the general alibi and the statement of interest that may indicate how credible the suspect's story from 1:00pm to $3: 00 \mathrm{pm}$ is. 


\section{Cues to Veracity}

We explored participants' self-reported cues to deception. Participants first gave an open-ended description of the verbal cues used to form their veracity judgement, and then identified which verbal cues they used from a set of predetermined cues (options: quantity of detail, verifiability of details, type of detail, consistency within the statement, logical structure/plausibility, clarity/vividness, or 'other'). The options included in this list were drawn from empirical findings regarding content- and strategy-based cues to deception (e.g., DePaulo et al., 2003; Johnson \& Raye, 1981; Steller \& Köhnken, 1989).

Coding. A wide variety of responses were obtained in response to the question: "What verbal cues did you use to decide whether the critical element of the interviewee's statement (1:00 to 3:00pm) was truthful or deceptive? " To condense these data, responses were assigned to specific verbal cues such as "Quantity of details", "Contradictions and inconsistencies", "Unexpected complications", or "Lack of conviction/memory". The complete list consisted of 41 cues and was derived from Criteria-Based Content Analysis (CBCA; Steller \& Köhnken, 1989) and Reality Monitoring (RM; Johnson \& Raye, 1981) literature as well as similar veracity-cue lists used by Bogaard and colleagues (2016, 2018), Vrij and colleagues (2006) and Akehurst and colleagues (1996). This coding scheme contained cues only regarding the content qualities of the statement and the speech characteristics that were identifiable in a written transcript. Additionally, a selection of data-driven verbal cues were included (e.g., "Differences between the baseline and critical element in terms of the type, or amount, of detail"). All responses were categorized according to the cues listed in Supplementary Table 12.

To establish reliability, two raters coded participants' open-ended responses for the presence of these cues. The agreement between raters was excellent (see Supplementary Table 10 for the exact values). After establishing reliability between the two raters, we considered only the cues that both raters were in agreement upon; therefore, we only coded a cue as present 
when both raters agreed upon its presence, when raters disagreed upon its presence the cue was scored as absent.

Supplementary Table 10

Reliability statistics for qualitative coding of verbal cues

\begin{tabular}{|c|c|c|c|c|}
\hline \multicolumn{2}{|c|}{ Verbal Cue } & \multirow{2}{*}{$\begin{array}{c}\text { Cohen's Kappa } \\
(\kappa)\end{array}$} & \multirow{2}{*}{$\begin{array}{c}\text { Significance } \\
\text { level }(p) \\
<.0005\end{array}$} & \multirow{2}{*}{$\begin{array}{c}\text { Percentage } \\
\text { Agreement } \\
100\end{array}$} \\
\hline 1. & Coherence & & & \\
\hline 2. & Clarity/vividness & 1.00 & $<.0005$ & 100 \\
\hline 3. & Spontaneous corrections & 0.949 & $<.0005$ & 99.33 \\
\hline 4. & Contradictions and inconsistency & 0.899 & $<.0005$ & 96.64 \\
\hline 5. & Perceptual information & 1.00 & $<.0005$ & 100 \\
\hline 6. & Emotions & 1.00 & $<.0005$ & 100 \\
\hline 7. & Quantity of details & 0.909 & $<.0005$ & 99.33 \\
\hline 8. & Spatial information & 1.00 & $<.0005$ & 100 \\
\hline 9. & Unstructured production & 0.878 & $<.0005$ & 97.99 \\
\hline 10. & Description of interaction & -- & -- & 100 \\
\hline 11. & Temporal information & 1.00 & $<.0005$ & 100 \\
\hline 12. & Extraneous information & 1.00 & $<.0005$ & 100 \\
\hline 13. & Reproduction of conversation & - & - & 99.33 \\
\hline 14. & Reconstructability of the statement & -- & -- & 100 \\
\hline 15. & Unusual details & 1.00 & $<.0005$ & 100 \\
\hline 16. & Plausibility & 0.902 & $<.0005$ & 98.66 \\
\hline 17. & Cognitive operations & 0.661 & $<.0005$ & 98.66 \\
\hline 18. & Denial of allegation & -- & -- & 100 \\
\hline 19. & Social introduction & 1.00 & $<.0005$ & 100 \\
\hline 20. & Lack of conviction or memory & 0.956 & $<.0005$ & 99.33 \\
\hline 21. & Main event of the statement & -- & -- & 100 \\
\hline 22. & Objective versus subjective time & 1.00 & $<.0005$ & 100 \\
\hline 23. & Missing information & 1.00 & $<.0005$ & 100 \\
\hline 24. & First person singular, past tense & - & - & 99.33 \\
\hline 25. & Use of pronouns & 1.00 & $<.0005$ & 100 \\
\hline 26. & Changes in language & 0.832 & $<.0005$ & 98.66 \\
\hline 27. & Length of the statement & -- & -- & 100 \\
\hline 28. & Self-references & -- & -- & 100 \\
\hline 29. & Grammatical errors/changes & 1.00 & $<.0005$ & 100 \\
\hline 30. & Repetitions & 0.892 & $<.0005$ & 99.33 \\
\hline 31. & Clichés & -- & -- & 100 \\
\hline 32. & Evasive responses & 1.00 & $<.0005$ & 100 \\
\hline 33. & Unexpected complications & 0.797 & $<.0005$ & 99.33 \\
\hline 34. & Self-deprecation & -- & -- & 100 \\
\hline 35. & NA/ nonsense response & 0.854 & $<.0005$ & 99.33 \\
\hline
\end{tabular}


36. Filler words indicative of

hesitations

37. Providing un/verifiable details

38. Differences between baseline and

critical period: Amount of detail

39. Differences between baseline and

critical period: Type of details

40. Specific details/wording/phrasing

$<.0005$

98.66

41. Sentence structure

Note. The reliability values are computed on the raw data from both raters. Both raters agreed on the complete absence of eight cues and the $k$ was not informative as both raters' scores were constant values (labelled as --). Additionally, in two cases, one of the two raters scored the value as absent and again $k$ was not informative (labelled as -). When $k$ was not informative, we instead report percentage agreement.

\section{Results}

We performed a content analysis to examine participants' open-ended responses regarding the cues they used to detect deception. Supplementary Table 11 provides an overview of the coding of participants' endorsement of these cues for the sample as a whole. The majority of participants reported using cues related to contradictions or inconsistencies, the presence of filler words, and the overall number of details. The fourth most frequently endorsed cue related to differences between the baseline and critical period, in terms of the type of detail (13\% of all reported cues). We were particularly interested in the verbal cues relating to comparisons between the general and critical elements of the statement. Accordingly, we conducted two chisquare tests of association between the two baseline conditions and their endorsement of the strategies relating to content-based differences (i.e., type or amount of detail) between the baseline and critical period. These analyses revealed that participants, across Baseline-present and Baseline-absent conditions, did not significantly differ in their use of cues related to differences between the baseline and critical period in terms of the amount of details or the type of details, $\chi^{2}(1)=.53, p=.467$ and $\chi^{2}(1)=1.51, p=.219$. Both associations were weak, $\varphi=.06$ and .10 , respectively. 
Supplementary Table 11

Participants' endorsement of verbal cues used for their veracity judgement

\begin{tabular}{|c|c|c|}
\hline Item & Total Frequency & Relative Percentage \\
\hline Contradictions or inconsistencies & 53 & 35.8 \\
\hline Filler words indicative of hesitation & 47 & 31.8 \\
\hline Quantity of details & 34 & 23.0 \\
\hline $\begin{array}{l}\text { Differences between baseline and critical period: } \\
\text { of detail }\end{array}$ & 19 & 12.8 \\
\hline Repetitions & 15 & 10.1 \\
\hline Lack of conviction or memory & 13 & 8.8 \\
\hline Sentence structure & 12 & 8.1 \\
\hline Unstructured production & 12 & 8.1 \\
\hline Changes in language & 12 & 8.1 \\
\hline Missing information & 12 & 8.1 \\
\hline Spontaneous corrections & 10 & 6.8 \\
\hline Plausibility & 10 & 6.8 \\
\hline Temporal information & 9 & 6.1 \\
\hline Verifiability of information & 8 & 5.4 \\
\hline $\begin{array}{l}\text { Differences between baseline and critical period: } \\
\text { Amount of detail }\end{array}$ & 8 & 5.4 \\
\hline Coherence & 7 & 4.7 \\
\hline Extraneous information & 7 & 4.7 \\
\hline Specific details or phrasing & 5 & 3.4 \\
\hline Use of pronouns & 5 & 3.4 \\
\hline Clarity/vividness & 4 & 2.7 \\
\hline Unusual details & 3 & 2.0 \\
\hline Evasive responses & 3 & 2.0 \\
\hline Nonsense or not applicable responses & 3 & 2.0 \\
\hline Cognitive operations & 2 & 1.4 \\
\hline Grammatical errors & 2 & 1.4 \\
\hline Unexpected complications & 2 & 1.4 \\
\hline Spatial information & 2 & 1.4 \\
\hline Perceptual information & 1 & 0.7 \\
\hline Emotions & 1 & 0.7 \\
\hline Social introduction & 1 & 0.7 \\
\hline Objective versus subjective time & 1 & 0.7 \\
\hline
\end{tabular}

Note. Although we used the 41 items in Appendix 2 to categorise the answers, only 31 different items of this list were present within the answers of our respondents.

We further explored participants' cue endorsement from the list of predetermined cues (see Supplementary Figure 2). The most frequently endorsed cue was "Consistency within the statement," reported by nearly $68 \%$ of participants. To test whether there was a difference in the endorsement of this cue between the two instruction groups, we conducted a chi-square test 
of independence, which revealed no statistically significant association between the groups, $\chi^{2}(1)=.49, p=.482$. This association was weak, $\varphi=.06$. Thus, participants across groups appear to have similarly relied on the consistency cue in their veracity decisions.

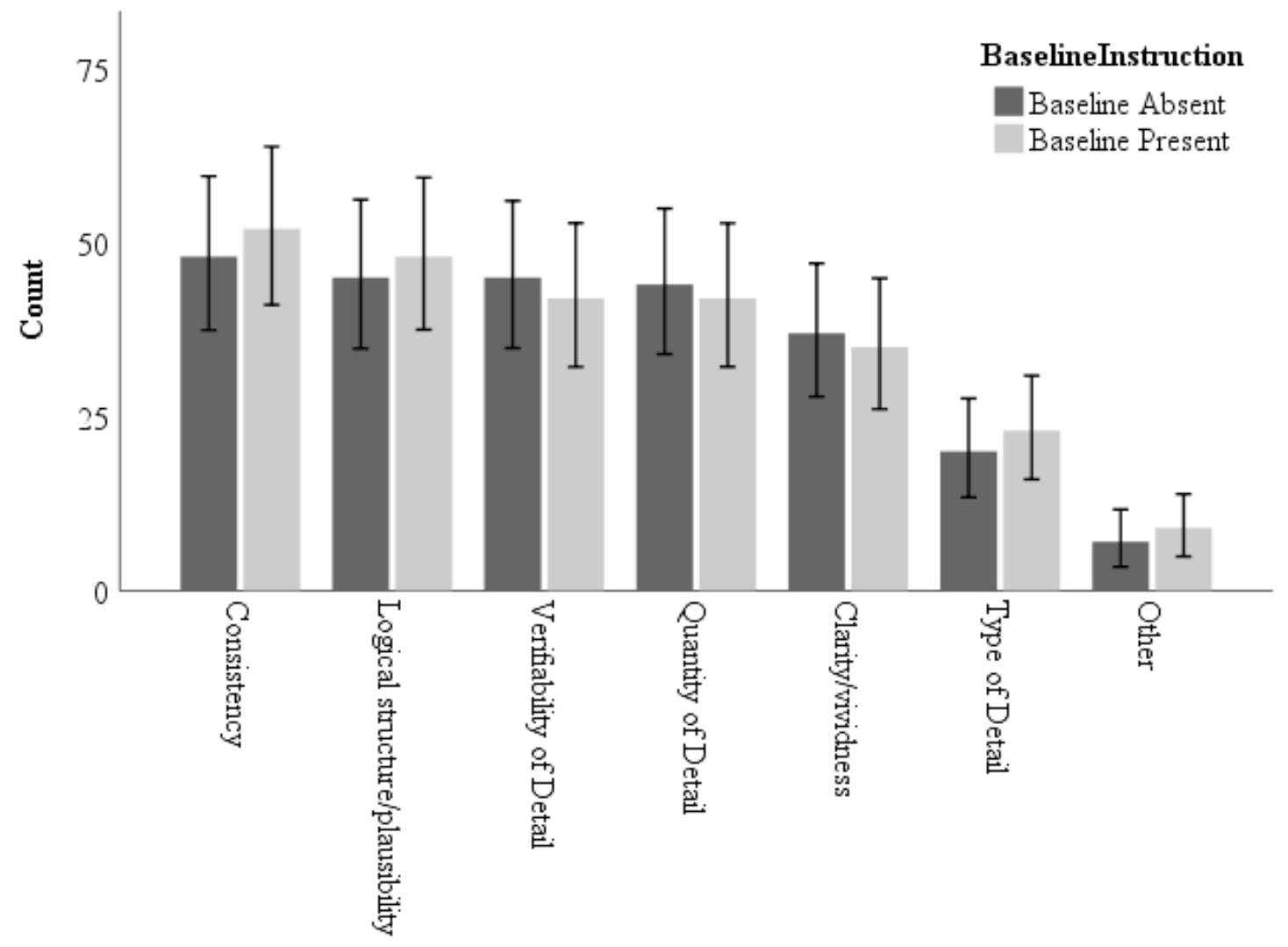

Verbal Cues to Veracity

Supplementary Figure 2. Participants' endorsement of veracity cues from a set of predetermined choices. Error bars represent $95 \%$ confidence intervals.

\section{Discussion}

Our qualitative data regarding participants' use of verbal cues of deception provided only a weak indication of the use of a comparable baseline. A small percentage of participants reported using cues related to content-based differences between the baseline and critical sections of the statements (i.e., $13 \%$ of the sample endorsed verbal cues relating to differences between the baseline and critical period in terms of the type of detail and $5 \%$ for the amount of detail). This could indicate that our baseline instruction was not strong enough to motivate 
participants' evaluation of statements based on verbal cues between the critical element and the comparable baseline section. It is also possible that the task of retrospectively reporting on higher-order cognitions was too challenging or perhaps the vague nature of our question led to less accurate responses (see Ericsson and Simon, 1980 for a discussion).

Supplementary Table 12

Descriptions and sources of verbal content cues

\begin{tabular}{|c|c|c|}
\hline Verbal Cue & Description & Source \\
\hline Coherence & $\begin{array}{l}\text { Refers to the extent to which all elements of } \\
\text { the statement are logical and make up a } \\
\text { unified whole. }\end{array}$ & CBCA \\
\hline $\begin{array}{l}\text { Contradictions and } \\
\text { inconsistency }\end{array}$ & $\begin{array}{l}\text { Refers to the extent to which a statement } \\
\text { contains elements that contradict each other. }\end{array}$ & CBCA \\
\hline Quantity of details & $\begin{array}{l}\text { Refers to whether a statement is rich in } \\
\text { detail and includes specific descriptions of } \\
\text { place, time, persons, objects, and events. }\end{array}$ & CBCA \\
\hline Unstructured production & $\begin{array}{l}\text { Refers to the chronological order in which } \\
\text { the statements is told. Are they told in the } \\
\text { same order in which they occurred } \\
\text { (chronological), or not (unstructured)? }\end{array}$ & CBCA \\
\hline Unexpected complications & $\begin{array}{l}\text { Refers to occurrences (caused by the } \\
\text { interviewee, a third person, or another } \\
\text { situational factor) that make a situation more } \\
\text { difficult than necessary, often characterised } \\
\text { by disrupted activity, failing efforts, failures } \\
\text { to progress and activity, suddenly appearing } \\
\text { people and/or obstacles, etcetera. }\end{array}$ & CBCA \\
\hline Unusual details & $\begin{array}{l}\text { Refers to details of people, objects, or events } \\
\text { that are unique, unexpected, or surprising } \\
\text { but meaningful in the context. }\end{array}$ & CBCA \\
\hline *Description of interaction & $\begin{array}{l}\text { Refers to how action and reaction of the } \\
\text { different actors presented in the statement } \\
\text { are linked. }\end{array}$ & CBCA \\
\hline * Reproduction of conversation & $\begin{array}{l}\text { Refers to whether parts of the conversation } \\
\text { are reported in original form or if the } \\
\text { different speakers are recognisable in the } \\
\text { reproduced dialogs. }\end{array}$ & CBCA \\
\hline Spatial information & $\begin{array}{l}\text { Refers to information about locations or the } \\
\text { spatial arrangement of people and/or objects. }\end{array}$ & CBCA, RM \\
\hline
\end{tabular}


Temporal information

Spontaneous corrections

Extraneous information

Lack of conviction or memory

Emotions

Clarity/vividness

Perceptual information

*Reconstructability of the statement

Plausibility

Cognitive operations

*Denial of allegation

Social introduction
Refers to information about when the event happened or explicitly describes a sequence of events.

Refers to corrections that are made or information that is added to material previously provided in the statement without having been prompted by the interviewer.

Refers to irrelevant details that are related to

CBCA, SCAN the event, but not necessary to understand the event.

Refers to when the writer is vague about CBCA, SCAN certain elements within the statement when the writer admits he or she has forgotten something.

Refers to information that describes how the participant felt during an event and how

CBCA, RM, these feelings develop and change throughout an event.

Refers to the extent to which a statement is clear, sharp, and vivid (instead of dim and vague).

Refers to the presence of sensory $\mathrm{RM}$ information in a statement. Does the statement include sensorial experiences such as sounds, smells, tastes, physical sensations, and visual details?

Refers to whether it is possible to reconstruct the event on the basis of the information given.

Refers whether the story is plausible and realistic and makes sense.

These cue descriptions of inferences are

$\mathrm{RM}$ made by the participant based on existing knowledge.

Refers to whether the examinee directly SCAN denies the allegation in the statement.

Refers to how the persons described in the SCAN statement are introduced. People that are described within a statement should be introduced in an unambiguous way, usually by mentioning their name and role. 

event.

Objective versus subjective time Refers to how different time periods are covered in the statement. Objective time refers to the actual duration of events described, whereas subjective time refers to the number of words used describing these events.

Missing information

Refers to phrases in the statement that SCAN indicate some information has been left out.

*First person singular, past tense Refers to the format in which a statement is SCAN written.

Use of pronouns

Refers to the use of pronouns in the SCAN statement.

Changes in language

Refers to the change of terminology or

SCAN vocabulary in the statement.

*Length of the statement

Refers to the length of the statement.

Literaturebased

*Self-references

Refers to the number of self-references made by the interviewee.

Literaturebased

Grammatical errors/changes

Refers to grammatical errors within the statement. Also refers to changes in

Literaturegrammar within the statement. based

Repetitions

Refers to the presence of word and or

Literaturesentence repetition. based

*Clichés

Refers to the presence of clichéd expressions within the statement.

Literaturebased

Evasive responses

Refers to the presence of evasive or indirect responses by the interviewee.

Literaturebased

*Self-deprecation

Refers to the presence of the interviewee undervaluing himself or being excessively

Literaturemodest. based

$\mathrm{NA} /$ nonsense response Refers to statements that are not applicable

Data-driven to the verbal cues used, or that don't make sense.

Filler words

Refers to the presence of filler words that Data-driven may indicate hesitation. 
Providing un/verifiable details Refers to the presence of verifiable or

Data-driven unverifiable (checkable/uncheckable) information.

Differences between baseline Refers to the presence of differences Data-driven and critical period: Amount of detail regarding the amount of detail included in the general statement compared to the critical element from 1:00-3:00pm.

Differences between baseline Refers to the presence of differences Data-driven and critical period: Type of details regarding the type of detail included in the general statement compared to the critical element from 1:00-3:00pm.

Specific details/wording /phrasing

Refers to the inclusion of specific Data-driven details/information or specific wording/phrasing. It also refers to a lack of specific detail/vagueness in the statement.

Sentence structure

Refers to changes to sentence structure. Also Data-driven refers to the way/style of reporting.

Note. The items marked with an asterisks (*) were included in the coding list but were scored as absent based on the coding of both raters. SCAN refers to Scientific Content Analysis (Sapir, 2005). 


\title{
Appendix 5 - Ethical Approval
}

The studies presented in this thesis were conducted under a research line granted to Brianna L. Verigin by the University of Maastricht's Ethical Committee for Psychology (ECP) in 2016. Below is the letter of favorable opinion for the research line under which each of the individual studies were conducted. ${ }^{11,12}$

\section{Maastricht University m Lending!}

\author{
Board of FPD \\ Universiteit Mastricht \\ Postbus 616 \\ 6200 MD Maastricht
}

Ethicar Review Cammittee

Pyycholagy and Neurascience

Our reference
ERCON-173_01_31_20t6

arect dai Mastriche

0031.43.369.4009 7-12-2016

Dear Board,

After examination of the research line entided "Where the Truth Lies: An Examination of how the Interaction of Truthful and Deceptive Elements within Statements can Faclitate Decegtion Detection", submitted by Brianna Verigin, the Ethical Review Committee Psychology and Neuroscience (ERCPN) came to the condusion that there are no objections to the execution of the research project as described in the said protocol with regard to the review framework used. The applicant has been informed that:

1. Approval has been granted for a period of flve vears, with the possibility to prolong.

2. If the approval has been granted for a research line, esch individual study within this line must be notified to the ERCPN using the form provided on the website. This does not include studies which are reviewed by a proposal committee (i.a. IMRI, EEG and TMS).

3. Changes to the aparoved research protocol must be submitted by the ERCPN.

4. The reference number should be mentioned in all correspondence with the ERCPN.

5. The reference number must be indicated on all advertising communications to recruit participants.

Yours sincerely,

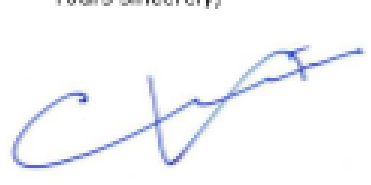

Prof. Dr. G. Kok Chair ERCPN

C. Brianna Verigin

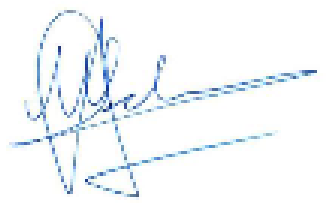

Mr. M. Schrijnemaekers Secretary ERCPN

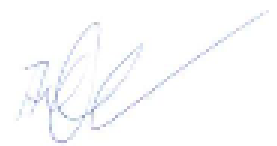

Prof. Dr. A.T. Sack Board of FPN

\footnotetext{
${ }^{11}$ The ethical approval of Maastricht University's ECP extends to cover the execution of a research project at the University of Portsmouth (Chapter 4; Study III).

${ }^{12}$ Each individual study within the research line received approval from Maastricht University ECP.
} 